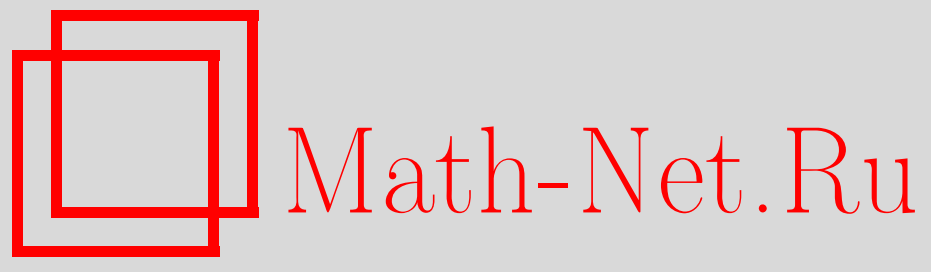

М. Фарбер, Д. Шютц, Замкнутые 1-формы в топологии и динамике, УМH, 2008, том 63, выпуск 6, 91-156

DOI: https://doi.org/10.4213/rm9259

Использование Общероссийского математического портала Math-Net.Ru подразумевает, что вы прочитали и согласны с пользовательским соглашением http://www . mathnet.ru/rus/agreement

Параметры загрузки:

IP: 3.85 .5 .30

26 апреля 2023 г., 12:33:34

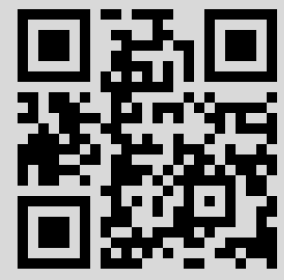




\section{Замкнутые 1-формы в топологии и динамике}

\section{М. Фарбер, Д. Шютц}

Настоящая статья является обзором недавних результатов по топологии и динамическим системам, основанных на технике замкнутых 1-форм. Наш подход позволяет делать выводы о свойствах потоков, изучая гомотопические и когомологические свойства многообразий. Более подробно мы описываем теорию типа Люстерника-Шнирельмана для замкнутых 1-форм, эффект фокусирования для потоков и теорию 1-форм Ляпунова. Мы также обсуждаем недавние результаты о когомологических оценках инвариантов $\operatorname{cat}(X ; \xi)$ и $\operatorname{cat}^{1}(X ; \xi)$ и их точные вычисления в некоторых примерах.

Библиография: 61 название.

\section{СоДЕРЖАНИЕ}

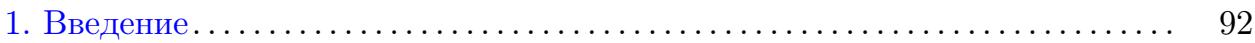

2. Основы теории Новикова................................. 94

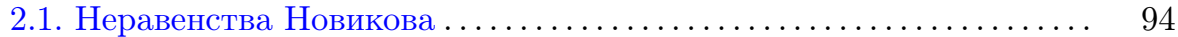

2.2. Комплекс Новикова и универсальный комплекс . . . . . . . . . . . . 97

2.3. Числа Новикова и фундаментальная группа ................. 99

3. Теорема о столкновении . . . . . . . . . . . . . . . . . . . . . 100

3.1. Слоения с особенностями, заданные замкнутыми 1-формами ... 101

3.2. Плотность слоев................................... 102

3.3. Модификация .................................... 103

3.4. Доказательство теоремы $3.1 \ldots \ldots \ldots \ldots \ldots \ldots \ldots \ldots \ldots \ldots \ldots . \ldots \ldots$

4. Замкнутые 1-формы на общих топологических пространствах ........ 107

4.1. Основные определения . . . . . . . . . . . . . . . . . . . . . 108

4.2. Интегрирование . . . . . . . . . . . . . . . . . . . . . . . . . . . . . . . . 108

4.3. Когомологический класс замкнутой 1-формы .............. 109

5. 1-формы Ляпунова для потоков .......................... 110

6. Понятие категории относительно когомологического класса . . . . . . . . . 117

6.1. Подвижные подмножества . . . . . . . . . . . . . . . . . . . . 118

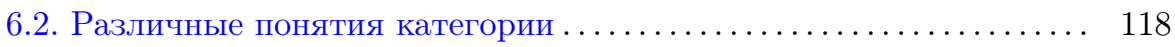

6.3. Гомотопическая инвариантность ..................... 121

6.4. Пространства категории нуль ........................ 121

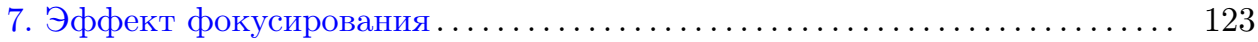

8. Существование окрестностей, выпуклых относительно потока ........ 127

(C) М. ФарьеР, Д. Шютц, 2008 


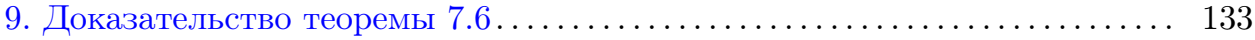

10. Топология цепно-рекуррентного множества $R_{\xi} \ldots \ldots \ldots \ldots \ldots \ldots \ldots \ldots$

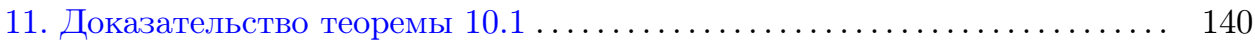

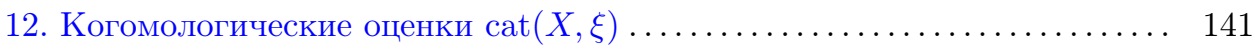

12.1. Понятие когомологической длины $\mathrm{cl}(X, \xi) \ldots \ldots \ldots \ldots \ldots \ldots . \ldots \ldots$

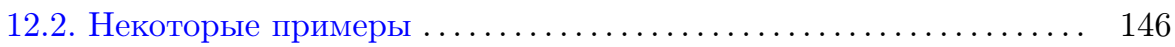

13. Верхние оценки для $\operatorname{cat}(X, \xi)$ и связь с инвариантами Бьери-Неймана-Штребеля . . . . . . . . . . . . . . . . . . . . . . . . . 148

14. Гомологические категорные веса, оценки для $\operatorname{cat}^{1}(X, \xi)$ и вычисление $\operatorname{cat}(X, \xi), \operatorname{cat}^{1}(X, \xi)$ для произведений поверхностей .......... 149

14.1. Гомологический категорный вес ....................... 149

14.2. Когомологическая оценка для $\operatorname{cat}^{1}(X, \xi) \ldots \ldots \ldots \ldots \ldots \ldots \ldots$

14.3. Категории $\operatorname{cat}(X, \xi)$ и $\operatorname{cat}^{1}(X, \xi)$ для произведений поверхностей 153

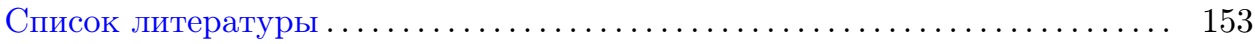

\section{1. Введение}

В 1981 г. С. П. Новиковым были инициированы исследования [1], [2], обобщающие теорию Морса, которые дают топологические оценки числа нулей замкнутых 1-форм (см. также [3], [4]). Мотивацией являлось наличие важных проблем математической физики, приводящих так или иначе к задаче нахождения соотношений между топологией многообразия и числом нулей замкнутой 1-формы из фиксированного одномерного класса вещественных когомологий. Напомним, что замкнутую 1-форму можно рассматривать как многозначную функцию или функционал, свойство ветвления которого полностью определяется классом когомологий.

В своей работе [2] Новиков изучал разные проблемы физики, где движение сводится к принципу экстремального действия некоторого многозначного функционала $S$ на пространстве кривых, вариация $\delta S$ которого является корректно определенной 1-формой (см. [5]-[7]). Среди проблем такого рода: уравнения Кирхгофа движения твердого тела в идеальной жидкости, уравнение Леггетта для магнитного момента и многие другие.

Основная идея Новикова [2] состояла в построении цепного комплекса, ныне называемого комплексом Новикова, с использованием динамики градиентного потока на абелевой накрывающей, ассоциированной с когомологическим классом. Динамика градиентных потоков, "перекидывающая мост" между множеством критических точек функции и глобальной объемлющей топологией многообразия, традиционно возникает в теории Морса.

В настоящее время теория Новикова замкнутых 1-форм является бурно развивающейся областью топологии, взаимодействующей со многими другими математическими теориями. Ж.-Кл. Сикорав [8], [9] первым применил теорию Новикова в симплектической топологии. Хоффер и Саламон [10] впервые использовали идеи теории Новикова в когомологиях Флоера. Более недавние применения теории Новикова в симплектической топологии и гамильтоновой динамике можно найти в работах Оха [11], Ушера [12] (см. также библиографию в этих работах). Другой областью, в которой теория Новикова играет важную 
роль, является комбинаторная теория групп. Эта связь также была обнаружена Сикоравом, который понял, что инварианты Бьери-Неймана-Штребеля могут быть выражены в терминах гомологий Новикова (в общем случае некоммутативных).

Многие задачи теории Новикова продолжают активно развиваться в современных исследованиях. В список этих задач входят:

а) построение цепных комплексов (более общих, чем комплекс Новикова), которые могли бы уловить связь между топологией многообразия и топологией множества нулей;

б) различные типы неравенств для замкнутых 1-форм (с условиями невырожденности типа Морса или Ботта);

в) эквивариантные неравенства и

г) проблемы точности этих неравенств.

Две недавние монографии [13], [14] дают описание теории замкнутых 1-форм под разными углами зрения.

Топология замкнутых 1-форм является более широкой областью исследований, которая вместе с теорией Новикова изучает и новую теорию типа Люстерника-Шнирельмана для 1-форм, начало которой было положено в 2002 г. в работе [15]. Целью последней теории является также нахождение связей между топологией множества нулей замкнутой 1-формы и гомотопической информацией, вытекающей в основном из свойств когомологического класса этой 1-формы. Выражением “типа Люстерника-Шнирельмана" мы хотим подчеркнуть, что не накладывается никаких условий на характер нулей формы - в отличие от теории Новикова, где требуется невырожденность всех нулей в смысле Mopca.

Хотя теория Люстерника-Шнирельмана для замкнутых 1-форм имеет много общего как с теорией Люстерника-Шнирельмана для функций, так и с теорией Новикова для замкнутых 1-форм, она в то же время сильно отличается от обеих этих классических теорий. Наиболее замечательный новый феномен это то, что в любом ненулевом классе когомологий всегда существует замкнутая 1-форма, имеющая не более одного нуля (см. теорему 3.1). Следовательно, новая теория - это теория не только о числе нулей, но и, как мы показываем в настоящей статье, о качественных динамических свойствах гладких потоков на многообразиях.

Имея гладкий поток, мы хотим “приручить" его с помощью замкнутой 1-формы, принадлежащей фиксированному классу когомологий. Более точно, мы хотим найти такую замкнутую 1-форму, чтобы поток был локально убывающим. Эта идея приводит к понятию замкнутой 1-формы Ляпунова, которое обобщает классическое понятие функции Ляпунова. Ключевой вопрос о существовании замкнутых 1-форм Ляпунова был решен в работах [16]-[20]. Заметим, что классическая теорема Конли [21], [22] дает ответ в частном случае нулевого класса когомологий, т. е. когда мы имеем дело с функциями Ляпунова. Если же когомологический класс ненулевой, то основную роль играет введенное Шварцманом [23], [24] понятие асимптотического цикла.

Краткое описание теории Люстерника-Шнирельмана для замкнутых 1-форм можно найти в десятой главе книги [13], опубликованной в 2004 г. Основной 
целью настоящей статьи является обзор новых результатов, полученных после 2004 г. Для полноты изложения мы начнем с описания основных результатов теории Новикова.

В готовящейся к публикации работе [25] мы вводим и изучаем сигма-инварианты конечного клеточного комплекса $X$, аналогичные сигма-инвариантам групп, введенным в [26], [27]. В этих инвариантах заключена информация о свойствах конечности для бесконечных абелевых накрытий комплекса $X$. Представленный обзор и препринт [25] объединяет общая идея: они основываются на свойствах подвижности подмножеств $X$ относительно замкнутой 1-формы, которые достаточно близки по духу гомологиям Новикова.

\section{2. Основы теории Новикова}

В работах [1], [2] С. П. Новиковым было предложено обобщение классической теории Морса, которое дает нижние оценки числа нулей замкнутых морсовских 1-форм. В этом разделе мы даем краткое изложение теории Новикова. Мы коснемся только следующих аспектов: неравенств Новикова, чисел Новикова и принципа Новикова, который приводит к понятию комплекса Новикова. За доказательствами и более подробной информацией читатель может обратиться к оригинальным статьям [1], [2] и монографии [13]. Историческую справку о развитии этих областей после работ [1], [2], а также библиографические ссылки можно найти в [13].

2.1. Неравенства Новикова. Пусть $M$ - гладкое многообразие. Замкнутая 1-форма $\omega$ на $M$ определяется как гладкое сечение кокасательного расслоения $T^{*} M \rightarrow M$, удовлетворяющее условию $d \omega=0$. По лемме Пуанкаре, для любой односвязной области $U \subset M$ существует гладкая функция $f_{U}: U \rightarrow \mathbb{R}$, определенная с точностью до локально постоянного слагаемого, для которой $\left.\omega\right|_{U}=d f_{U}$. Нулями формы $\omega$ являются такие точки $p \in M$, что $\omega_{p}=0$. Если $p$ лежит в односвязной области $U$, то $\omega_{p}=0$ тогда и только тогда, когда $p$ является критической точкой $f_{U}$.

ОПРЕДЕлЕНИЕ 2.1. Нуль $p \in M$ гладкой замкнутой 1-формы $\omega$ называется невырожденным, если он является невырожденной критической точкой для функции $f_{U}$.

Очевидно, что это свойство не зависит от выбора односвязной области $U$ и функции $f_{U}$.

ОПРедЕЛЕНИЕ 2.2. Индекс Морса невырожденного нуля $p$ формы $\omega$ определяется как индекс Морса точки $p$, рассматриваемой как критическая точка функции $\left.f\right|_{U}$.

Он принимает значения $0,1,2, \ldots, n$, где $n=\operatorname{dim} M$.

Основная задача теории Новикова формулируется следующим образом. Пусть $\omega$ - гладкая замкнутая 1-форма на замкнутом гладком многообразии $M$. Предположим дополнительно, что $\omega$ является морсовской, т. е. все ее нули невырождены в указанном выше смысле. Обозначим через $c_{i}(\omega)$ число нулей формы $\omega$, имеющих индекс $i$, где $i=0,1, \ldots, n$. Мы хотим оценить числа $c_{i}(\omega)$ 
в терминах топологии многообразия $M$ и заданного формой $\omega$ когомологического класса

$$
\xi=[\omega] \in H^{1}(M ; \mathbb{R}) .
$$

В случае классической теории Морса имеем $\xi=[\omega]=0$ (т.е. $\omega=d f$, где $f: M \rightarrow \mathbb{R}$ - некоторая гладкая функция) и ответ дают неравенства Морса

$$
c_{i}(\omega) \geqslant b_{i}(M)+q_{i}(M)+q_{i-1}(M),
$$

где $b_{i}(M)$ обозначает $i$-е число Бетти многообразия $M$, а $q_{i}(M)$ - минимальное число образующих в подгруппе кручения группы $H_{i}(M ; \mathbb{Z})$.

В работах [1], [2] С. П. Новиков ввел обобщения чисел $b_{i}(M)$ и $q_{i}(M)$, зависящие от класса когомологий $\xi$ формы $\omega\left(\right.$ см. (1)) и обозначаемые через $b_{i}(\xi)$ и $q_{i}(\xi)$ соответственно. Будем называть $b_{i}(\xi)$ числами Новикова-Бетти, а $q_{i}(\xi)-$ числами кручения Новикова. Их определения будут даны ниже. Неравенства Новикова в самой слабой своей форме утверждают следующее.

Теорема 2.3. Пусть $\omega-$ гладкая замкнутая 1-форма на гладком замкнутом многообразии М. Предположим, что все нули $\omega$ невырождены. Тогда

$$
c_{i}(\omega) \geqslant b_{i}(\xi)+q_{i}(\xi)+q_{i-1}(\xi),
$$

где $\xi=[\omega] \in H^{1}(M ; \mathbb{R})$ - класс когомологий формы $\omega$.

Заметим, что при $\xi=0$ число $b_{i}(\xi)$ совпадает с $b_{i}(M)$, а $q_{i}(\xi)$ совпадает с $q_{i}(M)$, т. е. неравенства Морса являются частным случаем неравенств Новикова.

Наша цель в этом разделе объяснить, не вникая в подробности, основные идеи доказательства теоремы 2.3. Полное доказательство читатель может найти в книге [13; с. 46-48].

Дадим определение кольца Новикова Nov $_{\mathbf{k}}$ над произвольным коммутативным кольцом k. Элементами $\mathbf{N o v}_{\mathbf{k}}$ являются "формальные степенные ряды" вида

$$
x=\sum_{\gamma \in \mathbb{R}} n_{\gamma} t^{\gamma},
$$

где $t$ - формальная переменная, коэффициентами являются элементы кольца $\mathbf{k}$, $n_{\gamma} \in \mathbf{k}$, а степени $\gamma \in \mathbb{R}$ - произвольные вещественные числа, удовлетворяющие условию: для любого $c \in \mathbb{R}$ множество

$$
\left\{\gamma \in \mathbb{R} ; n_{\gamma} \neq 0, \gamma>c\right\}
$$

конечно. Эквивалентно, элемент $x \in \mathbf{N o v}_{\mathbf{k}}$ может быть представлен в виде

$$
x=n_{0} t^{\gamma_{0}}+n_{1} t^{\gamma_{1}}+n_{2} t^{\gamma_{2}}+\cdots,
$$

где $n_{i} \in \mathbf{k}, \gamma_{i} \in \mathbb{R}, \gamma_{0}>\gamma_{1}>\gamma_{2}>\cdots$ и $\gamma_{i}$ стремится к $-\infty$. Другими словами, элементами кольца Новикова $\mathbf{N o v}_{\mathbf{k}}$ являются “лорановские" степенные ряды с целыми коэффициентами, степени которых стремятся к $-\infty$. Сложение в кольце $\mathbf{N o v}_{\mathbf{k}}$ задается сложением коэффициентов при одинаковых степенях $t$. Произведение в $\mathbf{N o v}_{\mathbf{k}}$ задается формулой

$$
\sum n_{\gamma} t^{\gamma} \cdot \sum n_{\gamma}^{\prime} t^{\gamma}=\sum n_{\gamma}^{\prime \prime} t^{\gamma}
$$


где

$$
n_{\gamma}^{\prime \prime}=\sum_{\gamma_{1}+\gamma_{2}=\gamma} n_{\gamma_{1}} n_{\gamma_{2}}^{\prime} .
$$

Заметим, что последняя сумма содержит только конечное число ненулевых слагаемых и, более того, множество степеней $\gamma \in \mathbb{R}$, для которых $n_{\gamma}^{\prime \prime}$ является ненулевым, обладает тем свойством, что множества (4) конечны.

Для упрощения обозначений вместо $\mathbf{N o v}_{\mathbb{Z}}$ будем использовать Nov.

Лемма 2.4. Колъцо Новикова Nov является кольцом главных идеалов. Более того, для любого поля $\mathbf{k}$ кольцо $\mathbf{N o v}_{\mathbf{k}}$ является полем.

Второе утверждение очевидно; доказательство первого можно найти в [13; c. 8]. Заслуга открытия леммы 2.4 в основном принадлежит С. П. Новикову, который обнаружил и сформулировал без доказательства похожий результат. Ее полные доказательства появились в работах [10], [28] и [13]. Аналогичные утверждения для колец рациональных функций имеются в работах [8], [29].

Теперь мы можем объяснить, как сопоставить классу когомологий $\xi \in$ $H^{1}(M ; \mathbb{R})$ числа Новикова $b_{i}(\xi)$ и $q_{i}(\xi)$. Предположим, что $M$ - гладкое замкнутое многообразие, хотя конструкция проходит и для конечных полиэдров. Рассмотрим групповое кольцо $\mathbb{Z}[\pi]$ фундаментальной группы многообразия $\pi=\pi_{1}\left(M, x_{0}\right)$. Класс когомологий $\xi$ задает кольцевой гомоморфизм

$$
\varphi_{\xi}: \mathbb{Z}[\pi] \rightarrow \text { Nov }
$$

который на элементах группы $g \in \pi$ определен формулой

$$
\varphi_{\xi}(g)=t^{\langle\xi, g\rangle} \in \text { Nov }
$$

Здесь $\langle\xi, g\rangle \in \mathbb{R}$ - значение класса когомологий $\xi$ на гомотопическом классе $g$. Напомним, что $t$ является формальной переменной в кольце Новикова. Очевидно, что

$$
\varphi_{\xi}\left(g g^{\prime}\right)=t^{\left\langle\xi, g g^{\prime}\right\rangle}=t^{\langle\xi, g\rangle+\left\langle\xi, g^{\prime}\right\rangle}=\varphi_{\xi}(g) \varphi_{\xi}\left(g^{\prime}\right),
$$

т. е. $\varphi_{\xi}$ мультипликативно на элементах группы. Следовательно, $\varphi_{\xi}$ по линейности продолжается на все групповое кольцо как кольцевой гомоморфизм. По хорошо известной конструкции, гомоморфизм (5) определяет над $M$ локальную систему левых Nov-модулей, которую будем обозначать через $\mathscr{L}_{\xi}$. Гомологии $H_{i}\left(M ; \mathscr{L}_{\xi}\right)$ этой локальной системы являются конечно порожденными модулями над кольцом Новикова Nov. Так как Nov - кольцо главных идеалов, то $H_{i}\left(M ; \mathscr{L}_{\xi}\right)$ разлагается в прямую сумму свободного модуля и Nov модуля кручения.

ОПРЕДЕЛЕНИЕ 2.5. Число Новикова-Бетти $b_{i}(\xi)$ определяется как ранг свободной части группы $H_{i}\left(X, \mathscr{L}_{\xi}\right)$. Число кручения Новикова $q_{i}(\xi)$ определяется как количество элементов в минимальной системе образующих подмодуля кручения группы $H_{i}\left(X ; \mathscr{L}_{\xi}\right)$.

Напомним определение гомологий с коэффициентами в локальной системе $H_{i}\left(M ; \mathscr{L}_{\xi}\right)$. Рассмотрим универсальное накрытие $\widetilde{M} \rightarrow M$ и комплекс сингулярных цепей $C_{*}(\widetilde{M})$. Последний является цепным комплексом свободных 
модулей над групповым кольцом $\mathbb{Z}[\pi]$. Кольцо Nov можно рассматривать как правый $\mathbb{Z}[\pi]$-модуль, где

$$
x \cdot g=x \varphi_{\xi}(g), \quad x \in \mathbf{N o v}, \quad g \in \pi .
$$

Тогда $H_{i}\left(M ; \mathscr{L}_{\xi}\right)$ - это гомологии цепного комплекса

$$
\operatorname{Nov} \otimes_{\mathbb{Z}[\pi]} C_{*}(\widetilde{M}) .
$$

2.2. Комплекс Новикова и универсальный комплекс. Основной идеей С.П. Новикова [1], [2], которая в итоге привела к теореме 2.3, является утверждение, что по любой замкнутой морсовской 1-форме $\omega$ на гладком замкнутом многообразии $M$ можно построить иепной комплекс $C^{\omega}$ (известный как комплекс Новикова), обладающий следующими двумя свойствами:

1) $C^{\omega}$ является цепным комплексом свободных Nov-модулей и у каждого модуля $C_{i}^{\omega}$ существует канонический базис, элементы которого находятся во взаимно однозначном соответствии с нулями формы $\omega$ индекса $i$, где $i \in\{0,1, \ldots, n=\operatorname{dim} M\} ;$

2) $C^{\omega}$ гомотопически эквивалентен комплексу $\mathbf{N o v} \otimes_{\mathbb{Z}[\pi]} C_{*}(\widetilde{M}) ;$ в частности, гомологии $H_{i}\left(C^{\omega}\right)$ изоморфны гомологиям $H_{i}\left(M ; \mathscr{L}_{\xi}\right)$.

Из этого утверждения, которое мы называем приниипом Новикова, очевидным образом следует теорема 2.3. Его можно сопоставить с классическим принизиом Морса, который утверждает, что для любой функиии Морса $f$ на замкнутом гладком многообразии $M$ существует иепной комплекс $C^{f}$ со следующими двумя свойствами:

1) $C^{f}$ является цепным комплексом свободных $\mathbb{Z}[\pi]$-модулей и у каждого модуля $C_{i}^{f}$ существует канонический базис, элементы которого находятся во взаимно однозначном соответствии с критическими точками функции $f$, имеющими индекс Морса $i$, где $i \in\{0,1, \ldots, n=\operatorname{dim} M\}$;

2) $C^{f}$ гомотопически эквивалентен цепному комплексу $C_{*}(\widetilde{M})$, где $\widetilde{M}-$ универсальное накрытие $M$, a $\pi=\pi_{1}(M)$ - фундаментальная группа $M$.

Из теории Морса известны разные конструкции, приводящие к комплексу $C^{f}$. Одна из них основывается на том, что многообразие $M$ допускает такое клеточное разбиение, в котором клетки находятся во взаимно однозначном соответствии с критическими точками функции $f$. Другая хорошо известная конструкция комплекса $C^{f}$ (над полем действительных чисел) основана на виттеновской деформации комплекса де Рама.

Естественно возникает вопрос о существовании отличных от (5) кольцевых гомоморфизмов

$$
\rho: \mathbb{Z}[\pi] \rightarrow \mathscr{R},
$$

для которых имеет место аналог принципа Новикова. Точнее, будем говорить, что принцип Новикова верен для группы $\pi$, группового гомоморфизма ${ }^{1}$ $\xi: \pi \rightarrow \mathbb{R}$ и кольцевого гомоморфизма $\rho: \mathbb{Z}[\pi] \rightarrow \mathscr{R}$, если для любого гладкого замкнутого многообразия $M$ с фундаментальной группой $\pi_{1}(M)=\pi$ и любой замкнутой морсовской 1-формы $\omega$ на $M$, представляющей класс когомологий $\xi$, существует клеточный комплекс $C^{\omega}$ свободно порожденных $\mathscr{R}$-модулей,

$$
C^{\omega}=\left(0 \rightarrow C_{n}^{\omega} \rightarrow C_{n-1}^{\omega} \rightarrow \cdots \rightarrow C_{1}^{\omega} \rightarrow C_{0}^{\omega} \rightarrow 0\right),
$$

\footnotetext{
${ }^{1} \xi\left(g g^{\prime}\right)=\xi(g)+\xi\left(g^{\prime}\right)$ для $g, g^{\prime} \in \pi$.
} 
со следующими двумя свойствами:

1) в каждом модуле $C_{i}^{\omega}$ можно выбрать канонический базис так, чтобы элементы базиса находились во взаимно однозначном соответствии с нулями индекса $i$ формы $\omega$;

2) комплекс $C^{\omega}$ гомотопически эквивалентен комплексу $\mathscr{R} \otimes_{\mathbb{Z}[\pi]} C_{*}(\widetilde{M})$, где $\mathscr{R}$ рассматривается как левый $\mathbb{Z}[\pi]$-модуль над $\rho$.

Положительный ответ на этот вопрос для рационального класса когомологий дан в работе [30], а для общего случая - в работе [31]. Помимо гомоморфизма (5), принцип Новикова имеет место и для многих других кольцевых гомоморфизмов (7). Более того, среди таких гомоморфизмов есть "максимальный", который мы опишем ниже. Цепной комплекс $C^{\omega}$ в этом случае называется универсальным комплексом. Этот комплекс "живет" над локализацией группового кольца $\mathbb{Z}[\pi]$.

Элемент $\alpha \in \mathbb{Z}[\pi]$ будем называть $\xi$-отрицательным, если $\alpha=\sum n_{j} g_{j}$ (конечная сумма), где $n_{j} \in \mathbb{Z}$ и $\xi\left(g_{j}\right)<0$ для всех $j$. Матрица $A$ размера $m \times m$ над групповым кольцом $\mathbb{Z}[\pi]$ называется $\xi$-отрицательной, если все ее элементы $\xi$-отрицательны. Рассмотрим множество $\Sigma_{\xi}$ квадратных матриц вида $I+A$, где $A$ - произвольная $\xi$-отрицательная квадратная матрица с элементами из $\mathbb{Z}[\pi]$.

Заметим, что в случае $\xi=0$ (т. е. для морсовских функций) множество $\xi$-отрицательных матриц пусто и поэтому локализация Кона (8) является тождественным отображением.

Мы будем пользоваться понятием некоммутативной локализации, развитым Коном [32]. Универсальная локализащия Кона группового кольца $\mathbb{Z}[\pi]$ относительно класса $\Sigma_{\xi}-$ это кольцо $\Sigma_{\xi}^{-1}(\mathbb{Z}[\pi])$ вместе с кольцевым гомоморфизмом

$$
\rho_{\xi}: \mathbb{Z}[\pi] \rightarrow \Sigma_{\xi}^{-1}(\mathbb{Z}[\pi]),
$$

удовлетворяющее следующим двум условиям: во-первых, любая матрица вида $\rho_{\xi}(I+A)$, где матрица $A$ является $\xi$-отрицательной, над кольцом $\Sigma_{\xi}^{-1}(\mathbb{Z}[\pi])$ и, во-вторых, существует универсальный гомоморфизм с этим свойством, т. е. для любого кольцевого гомоморфизма $\rho: \mathbb{Z}[\pi] \rightarrow \mathscr{R}$, обращающего все матрицы вида $I+A$ обратимы (где $A$ является $\xi$-отрицательной), существует единственный кольцевой гомоморфизм $\varphi: \Sigma_{\xi}^{-1}(\mathbb{Z}[\pi]) \rightarrow \mathscr{R}$, для которого коммутативна диаграмма

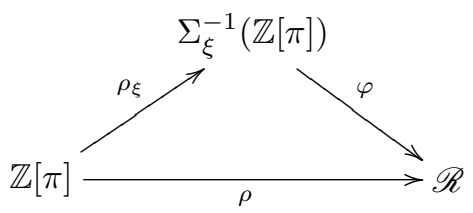

Существование локализации (8) доказано в книге Кона [32].

ТЕОРема 2.6. Пусть $\pi$ - произвольная группа $и \xi \in H^{1}(\pi ; \mathbb{R})-$ класс когомологий. Тогда принщип Новикова верен для локализачии Кона (8).

В работе [30] эта теорема доказана для целочисленных классов когомологий; общий случай рассмотрен в [33]. Конструкция универсального комплекса $C^{\omega}$ использует технику стягивания для цепных комплексов, которая аналогична 
хорошо известному комбинаторному стягиванию. Эта техника появилась в работе [30].

Обратим внимание на одно важное и тесно связанное с кольцом Новикова кольцо, называемое пополнением Новикова-Сикорава $\widehat{\mathbb{Z} \pi_{\xi}}$; оно было введено Сикоравом [8], который действовал в духе конструкции кольца Новикова Nov. Кольцо $\widehat{\mathbb{Z} \pi_{\xi}}$ можно рассматривать как некоммутативный аналог кольца Новикова. Элементами $\widehat{\mathbb{Z} \pi_{\xi}}$ являются формальные (возможно, бесконечные) суммы

$$
\alpha=\sum n_{i} g_{i}, \quad n_{i} \in \mathbb{Z}, \quad g_{i} \in \pi,
$$

удовлетворяющие условию: для любого $c \in \mathbb{R}$ множество $\left\{i ; \xi\left(g_{i}\right) \geqslant c\right\}$ конечно. Сравним это с конструкцией кольца Новикова. Сложение и умножение задаются обычными формулами; например, произведение элементов

$$
\alpha=\sum n_{i} g_{i} \in \widehat{\mathbb{Z} \pi_{\xi}} \quad \text { и } \quad \beta=\sum m_{j} h_{j} \in \widehat{\mathbb{Z} \pi_{\xi}}
$$

задается формулой

$$
\alpha \cdot \beta=\sum_{i, j}\left(n_{i} m_{j}\right)\left(g_{i} h_{j}\right) .
$$

Кольцевой гомоморфизм $\rho: \mathbb{Z}[\pi] \rightarrow \widehat{\mathbb{Z} \pi_{\xi}}$ является вложением. Если $A$ есть $\xi$-отрицательная квадратная матрица над кольцом $\mathbb{Z}[\pi]$, то степенной ряд

$$
(I+A)^{-1}=I-A+A^{2}-\cdots
$$

сходится в $\widehat{\mathbb{Z} \pi_{\xi}}$ и, следовательно, матрица $I+A$ обратима в кольце $\widehat{\mathbb{Z} \pi_{\xi}}$. Из универсального свойства локализации Кона следует существование канонического кольцевого гомоморфизма

$$
\Sigma_{\xi}^{-1}(\mathbb{Z}[\pi]) \rightarrow \widehat{\mathbb{Z} \pi_{\xi}}
$$

продолжающего вложение $\mathbb{Z}[\pi] \rightarrow \widehat{\mathbb{Z} \pi_{\xi}}$. Отсюда следует инъективность гомоморфизма (8).

Неизвестно, всегда ли гомоморфизм (10) является инъективным. Интуитивно, образ (10) состоит из "рационалъных степенных рядов". Это следствие характеризации элементов локализации Кона как компонент решений линейной системы уравнений; см. [32; гл. 7].

2.3. Числа Новикова и фундаментальная группа. Очевидно, что одномерные числа Новикова $b_{1}(\xi)$ и $q_{1}(\xi)$ зависят только от фундаментальной группы $\pi_{1}(X)$ и гомоморфизма периодов $\xi: \pi_{1}(X) \rightarrow \mathbb{R}$. Интересно, что существует и сильная обратная зависимость, а именно, информацию о фундаментальной группе иногда можно восстановить по числам $b_{1}(\xi)$ и $q_{1}(\xi)$. Следующий результат показывает, что первое число Новикова-Бетти $b_{1}(\xi)$ может быть ненулевым только для “больших" фундаментальных групп $\pi_{1}(X)$.

TЕОРема 2.7 (см. [34]). Пусть $X$ - конечный свлзный комплекс, и пусть $\xi \in H^{1}(X ; \mathbb{R})$ - ненулевой класс когомологий. Если первое число НовиковаБетти $b_{1}(\xi)$ положительно, то фундаментальная группа $\pi_{1}(X)$ содержит свободную подгруппу. 
Из теоремы 2.7 вытекают следующие утверждения.

СледСтвиЕ 2.8. Пусть $X$ - конечный связный полиэдр с аменабельной фундаментальной группой. Тогда первое число Новикова-Бетти $b_{1}(\xi)$ равно нулю для любого класса когомологий $\xi \neq 0 \in H^{1}(X ; \mathbb{R})$.

СлеДСтвиЕ 2.9. Пусть $X$ - конечный связный двумерный полиэдр. Если эйлерова характеристика $X$ отрицательна, $\chi(X)<0$, то фундаментальная группа $\pi_{1}(X)$ содержит свободную подгруппу.

Следствие 2.9 вытекает из теоремы 2.7 и теоремы Эйлера-Пуанкаре для чисел Новикова-Бетти:

$$
\sum_{i=0}^{\infty}(-1)^{i} b_{i}(\xi)=\chi(X) .
$$

Доказательство теоремы 2.7 дано в статье [34] и основывается на результатах работ [26] и [8].

Кроме того, из следствия 2.9 вытекает

СледСтвиЕ 2.10. Любая дискретная группа $G$ дефекта $>1$ содержит неабелеву свободную подгруппу.

Следствие 2.10, отвечающее на один вопрос Р. Линдона из комбинаторной теории групп, было ранее получено Н. С. Романовским ${ }^{2}$ с помощью чисто алгебраического метода. Наше доказательство является геометрическим и полностью независимым от доказательства Романовского.

Дополнительную информацию читатель может найти в статьях [35]-[38].

\section{3. Теорема о столкновении}

Как следует из предыдущего раздела, теория Новикова дает нижнюю оценку количества различных нулей замкнутой морсовской 1-формы $\omega$, принадлежащей заданному классу когомологий $\xi \in H^{1}(M ; \mathbb{R})$. Общее количество нулей не меньше чем сумма $\sum_{j} b_{j}(\xi)$ чисел Новикова-Бетти $b_{j}(\xi)$.

Если замкнутая 1-форма $\omega$ представляет нулевой класс когомологий, то $\omega=d f$, где $f: M \rightarrow \mathbb{R}$ - гладкая функция; в этом случае, согласно классической теории Люстерника-Шнирельмана [39], у формы $\omega$ должно быть не менее $\operatorname{cat}(M)$ геометрически различных нулей (которые суть в точности критические точки функции $f$ ); этот результат не требует дополнительных предположений о характере нулей.

После столь успешного развития теории Новикова возникает естественный вопрос: возможно ли построение аналога теории Люстерника-Шнирельмана для замкнутых 1-форм? Удивительно, что в общем случае, за исключением двух упомянутых выше ситуаций, нет препятствий $\kappa$ построению замкнутой 1-формы с единственным нулем. Следовательно, при $\xi \neq 0$ единственный гомотопический инвариант $a(M, \xi)$ (зависящий от топологии многообразия $M$ и когомологического класса $\xi)$ такой, что любая замкнутая 1-форма $\omega$ на $M$, представляющая класс $[\omega]=\xi$, имеет не более чем $a(M, \xi)$ нулей, есть $a(M, \xi)=0$ или $a(M, \xi)=1$.

\footnotetext{
${ }^{2}$ Н. С. Романовский, “Свободные подгруппы в конечно-определенных группах”, Алгебра и логика, 16:1 (1977), 88-97.
} 
ТеОРема 3.1 [15], [40]. Пусть $M$ - замкнутое, связное, гладкое $n$-мерное многообразие и $\xi \in H^{1}(M ; \mathbb{R})$ - вещественный ненулевой класс когомологий. Тогда существует гладкая замкнутая 1-форма $\omega$ из класса $\xi$ с не более чем одним нулем.

Этот результат подсказывает, что “теория Люстерника-Шнирельмана для замкнутых 1-форм" (если она существует) должна существенно отличаться как от классической теории Люстерника-Шнирельмана, так и от теории Новикова для замкнутых 1-форм.

Теорема 3.1 была доказана в работе [15] при дополнительном предположении, что класс $\xi$ является целочисленным, $\xi \in H^{1}(M ; \mathbb{Z})$ (см. также [13; теорема 10.1]). В полной общности теорема была доказана в [40].

Коснемся кратко аналогичного вопроса. Известно, что если эйлерова характеристика многообразия нулевая, $\chi(M)=0$, то на $M$ существует всюду ненулевая 1-форма $\omega$. При $\chi(M)=0$ можно задать вопрос о существовании на многообразии $M$ всюду ненулевой 1-формы $\omega$, которая является замкнутой, т. е. $d \omega=0$. В общем случае ответ отрицательный. Например, равенство нулю чисел Новикова-Бетти, $b_{j}(\xi)=0$, является необходимым условием существования в классе когомологий $\xi$ представителя без критических точек. Полный перечень необходимых и достаточных условий (при $\operatorname{dim} M>5$ ) дает теорема Латура [41].

Теорема 3.1 была доказана в [15] и [13] в случае, когда ранг когомологического класса $\xi$ равен 1. В этом разделе мы дадим доказательство теоремы 3.1 для случая $\operatorname{rk}(\xi)>1$, следуя нашей недавней работе [40].

\section{1. Слоения с особенностями, заданные замкнутыми 1-формами.} Пусть $M$ - гладкое многообразие. Гладкая замкнутая 1-форма $\omega$ с морсовскими особенностями задает на многообразии $M$ слоение с особенностями $\omega=0$. Это есть разбиение многообразия $M$ на слои: две точки $p, q \in M$ принадлежат одному и тому же слою тогда и только тогда, когда существует путь $\gamma:[0,1] \rightarrow M$ с концами в этих точках: $\gamma(0)=p, \gamma(1)=q$, и значение формы $\omega$ на векторе скорости $\gamma$ равно нулю: $\omega(\dot{\gamma}(t))=0$ для всех $t$. Локально, в односвязной области $U \subset M$, имеем $\left.\omega\right|_{U}=d f$ для некоторой гладкой функции $f: U \rightarrow \mathbb{R} ;$ любая компонента связности поверхности уровня $f^{-1}(c)$ принадлежит одному слою. Если окрестность $U$ достаточно мала и не содержит нулей $\omega$, то на $U$ можно задать такую систему координат $x_{1}, \ldots, x_{n}$, для которой $f \equiv x_{1}$; следовательно, слоями в окрестности $U$ являются множества $x_{1}=c$. Вблизи таких точек слоение с особенностями $\omega=0$ является обычным слоением. И наоборот, если $U$ есть малая окрестность нуля $p \in M$ формы $\omega$ и индекс Морса этой точки равен $k, 0 \leqslant k \leqslant n$, то на $U$ существует такая система координат $x_{1}, \ldots, x_{n}$, что $x_{i}(p)=0$ и слои слоения с особенностями $\omega=0$ в $U$ являются множествами уровня $-x_{1}^{2}-\cdots-x_{k}^{2}+x_{k+1}^{2}+\cdots+x_{n}^{2}=c$. Слой $L$ при $c=0$ содержит нуль $p$. Этот слой имеет особенность в точке $p$ : окрестность $p$ в слое $L$ гомеоморфна конусу над произведением $S^{k-1} \times S^{n-k-1}$. Существует только конечное число особых слоев, т. е. слоев, содержащих нули формы $\omega$.

Нас особенно интересуют особые слои, содержащие нули индексов 1 и $n-1$ 1 -формы $\omega$. После удаления такой точки $p$ слой $L$ становится локалъно несвяз- 
ным. Однако глобально дополнение к $L-\{p\}$ может быть как связным, так и несвязным.

Слоение с особенностями $\omega=0$ является коориентированным: нормальное расслоение к каждому слою в каждой неособой точке имеет определенную ориентацию.

Мы будем пользоваться введенным Левитом [42] понятием слабо полных замкнутых 1-форм. Замкнутая 1-форма $\omega$ называется слабо полной, если все ее критические точки морсовские и для любого гладкого пути $\sigma:[0,1] \rightarrow M^{*}$ с $\int_{\sigma} \omega=0$ концы $\sigma(0)$ и $\sigma(1)$ принадлежат одному и тому же слою слоения $\omega=0$ на $M^{*}$. Здесь через $M^{*}$ обозначено множество $M-\left\{p_{1}, \ldots, p_{m}\right\}$, где $p_{j}-$ нули формы $\omega$.

У слабо полных замкнутых 1-форм из ненулевого класса когомологий $\xi=$ $[\omega] \neq 0$ нет нулей индексов 0 и $n$. Согласно [42], в любом ненулевом классе когомологий $\xi \in H^{1}(M ; \mathbb{R})$ можно найти слабо полного представителя.

Приведем план нашего доказательства теоремы 3.1. Мы начнем со слабо полной замкнутой 1-формы $\omega$ из данного класса когомологий $\xi \in H^{1}(M ; \mathbb{R})$, $\xi \neq 0$. Мы покажем, что в предположении $\operatorname{rk}(\xi)>1$ все слои слоения с особенностями $\omega=0$ являются всюду плотными (см. п. 3.2). Возмущением формы $\omega$ получим замкнутую 1-форму $\omega^{\prime}$ с единственным особым слоем (см. п. 3.3). После этого мы применим технику Такенса [43], которая позволит нам собрать все нули на особом слое в одну (сильно вырожденную) точку. Сначала докажем теорему 3.1 при $n=\operatorname{dim} M>2$; специальный случай $n=2$ будет рассмотрен в конце п. 3.4.

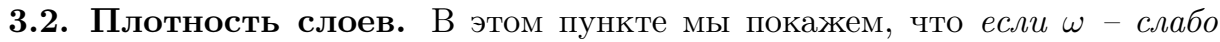
плотная форма $u \operatorname{rk}(\xi)>1$, то слои слоения с особенностями $\omega=0$ являются плотными; более того, для данной точки $x \in M$ и данного слоя $L \subset M$ слоения с особенностями $\omega=0$ существуют две последовательности точек $x_{k} \in L$ $u y_{k} \in L$ maкue, что $x_{k} \rightarrow x, y_{k} \rightarrow x u$

$$
\int_{x}^{x_{k}} \omega>0, \quad \int_{x}^{y_{k}} \omega<0
$$

(интеграл в формуле (11) вычисляется вдоль произвольного пути из малой окрестности $x$ ). Другими словами, можно сказать, что любой слой $L$ приближается к точке $x$ с положительной и отрицательной стороны. Этот факт также был отмечен Левитом [42; с. 645]; мы дадим ниже другое доказательство. В общем случае для форм без минимаксов и с условием $\operatorname{rk}(\xi)>1$ утверждение о плотности слоев слоения $\omega=0$ является неверным (см. примеры в книге [13; гл. $11, \S 9.3]$.

Пусть $\omega$ - слабо полная, замкнутая 1-форма, задающая класс когомологий $\xi$. Рассмотрим накрытие $\pi: \widetilde{M} \rightarrow M$, соответствующее ядру гомоморфизма периодов $\operatorname{Per}_{\xi}: \pi_{1}(M) \rightarrow \mathbb{R}$, где $\xi=[\omega] \in H^{1}(M ; \mathbb{R})$. Пусть $H \subset \mathbb{R}-$ группа периодов. Ранг группы $H$ совпадает $\mathrm{c} \operatorname{rk}(\xi)$ над полем рациональных чисел; поскольку по предположению $\operatorname{rk}(\xi)>1$, подгруппа $H$ плотна в $\mathbb{R}$. Группа периодов $H$ действует на накрывающей $\widetilde{M}$ как группы трансляций накрытия. Для 1-формы $\pi^{*} \omega$ существует гладкая функция $F: \widetilde{M} \rightarrow \mathbb{R}$ такая, что $\pi^{*} \omega=d F$. Слои слоения с особенностями $\omega=0$ являются образами множеств 


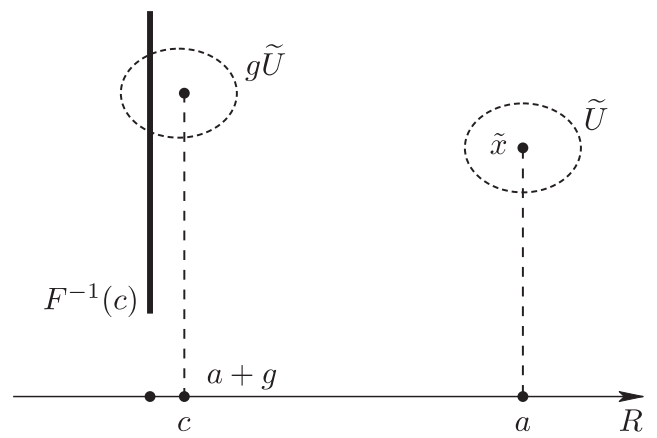

Рис. 1

уровня $F^{-1}(c)$ относительно проекции $\pi$; это свойство следует из слабой полноты формы $\omega$ (см. [42; предложение II.1]). Для любых $g \in H$ и $x \in \widetilde{M}$ имеем

$$
F(g x)-F(x)=g \in \mathbb{R} .
$$

Пусть $L=\pi\left(F^{-1}(c)\right)$ - слой и $x \in M-$ произвольная точка. Наша цель показать, что точка $x$ лежит в замыкании $\bar{L}$ слоя $L$. Пусть $U \subset M-$ линейно связная окрестность точки $x$, которая предполагается достаточно "маленькой" в следующем смысле: $\left.\xi\right|_{U}=0$. Мы хотим показать, что $U \cap L$ содержит та-

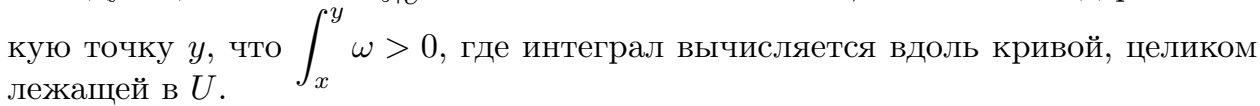

Рассмотрим поднятие $\tilde{x} \in \widetilde{M}, \pi(\tilde{x})=x$. Пусть $\widetilde{U}$ - такая окрестность точки $\tilde{x}$, которая гомеоморфно отображается на $U$ при проекции $\pi$. Мы утверждаем, что множество значений $F(\widetilde{U}) \subset \mathbb{R}$ содержит интервал $(a-\varepsilon, a+\varepsilon)$, где $a=F(\tilde{x}) u \varepsilon>0$. Это утверждение очевидно, если $\tilde{x}$ не является критической точкой функции $F$, поскольку в этом случае в окрестности точки $\tilde{x}$ можно выбрать такую систему координат $x_{1}, \ldots, x_{n}$, что $F(x)=a+x_{1}$. Когда $\tilde{x}$ - критическая точка функции $F$, в окрестности $\tilde{x}$ можно выбрать систему координат $x_{1}, \ldots, x_{n}$, в которой функция $F$ имеет вид $a \pm x_{1}^{2} \pm x_{2}^{2} \pm \cdots \pm x_{n}^{2}$. Наше утверждение следует из того, что индекс Морса этой критической точки отличен от 0 и $n$.

Ввиду плотности группы трансляций $H \subset \mathbb{R}$ можно найти такой элемент $g \in H$, что вещественное число $F(g \tilde{x})=F(\tilde{x})+g=a+g$ принадлежит интервалу $(c, c+\varepsilon)$. Таким образом, получаем

$$
c \in(a+g-\varepsilon, a+g) \subset g+F(\widetilde{U})=F(g \widetilde{U}) .
$$

Мы видим, что множества $F^{-1}(c)$ и $g \widetilde{U}$ имеют общую точку $\tilde{y}$ (ср. рис. 1$)$. Эта точка $y=\pi(\tilde{y}) \in U \cap L$ удовлетворяет требуемому условию $\int_{x}^{y} \omega>0$.

3.3. Модификация. Нашей следующей задачей является замена формы $\omega$ на морсовскую замкнутую форму $\omega^{\prime}$, все критические точки которой лежат на одном особом слое слоения с особенностями $\omega^{\prime}=0$. В этом пункте мы предполагаем, что $n=\operatorname{dim} M>2$. 


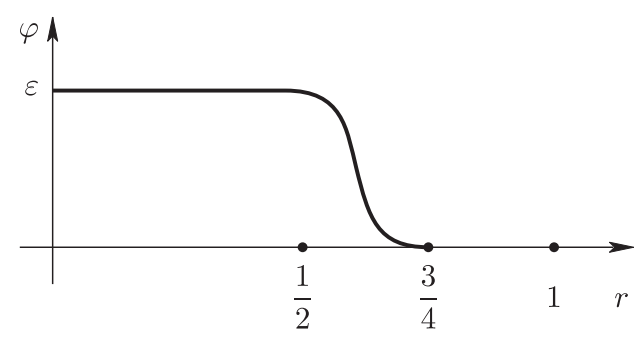

Рис. 2

Пусть $\omega$ - слабо полная морсовская замкнутая 1-форма, задающая иррациональный класс когомологий $\xi$, т. е. $\operatorname{rk}(\xi)>1$. Пусть $p_{1}, \ldots, p_{m} \in M-$ нули формы $\omega$. Для каждого $p_{j}$ выберем малую окрестность $U_{j} \ni p_{j}$ и локальные координаты $x_{1}, \ldots, x_{n}$ в $U_{j}$ так, что $x_{i}\left(p_{j}\right)=0$ для всех $i=1, \ldots, n$ и

$$
\left.\omega\right|_{U_{j}}=d f_{j}, \quad \text { где } f_{j}=-x_{1}^{2}-\cdots-x_{m_{j}}^{2}+x_{m_{j}+1}^{2}+\cdots+x_{n}^{2} .
$$

Здесь $m_{j}$ обозначает индекс точки $p_{j}$. Предположим, что шар $\sum_{i=1}^{n} x_{i}^{2} \leqslant 1$ содержится в $U_{j}$ и $U_{j} \cap U_{j^{\prime}}=\varnothing$ при $j \neq j^{\prime}$. Обозначим через $W_{j}$ открытый шар $\sum_{i=1}^{n} x_{i}^{2}<1$.

Пусть $\varphi:[0,1] \rightarrow[0,1]$ - гладкая функция, удовлетворяющая следующим условиям: (a) $\varphi \equiv 0$ на $[3 / 4,1] ;$ (b) $\varphi \equiv \varepsilon>0$ на $[0,1 / 2] ;$ (c) $-1<\varphi^{\prime} \leqslant 0$ (ср. рис. 2). Такая функция существует для любого достаточно малого $\varepsilon>0$. Из условий (а)-(c) следует, что

$$
\varphi^{\prime}(r)>-2 r \quad \text { при } r>0 .
$$

Заменим замкнутую 1-форму $\omega$ на

$$
\omega^{\prime}=\omega-\sum_{j=1}^{m} \mu_{j} \cdot d g_{j}
$$

где $g_{j}: M \rightarrow \mathbb{R}$ - гладкая функция с носителем в $U_{j}$. В координатах $x_{1}, \ldots, x_{n}$ на $U_{j}$ (см. выше) функция $g_{j}$ задается формулой $g_{j}(x)=\varphi(\|x\|)$. Параметры $\mu_{j} \in[-1,1]$ из формулы (16) будут уточнены позднее.

Имеем $\omega \equiv \omega^{\prime}$ на $M-\bigcup_{j} U_{j}$ и около нулей формы $\omega$. Покажем, что у формы $\omega^{\prime}$ нет дополнительных нулей. Очевидно, что $\left.\omega^{\prime}\right|_{U_{j}}=d\left(f_{j}-\mu_{j} g_{j}\right)$ (где $f_{j}$ определены в (14)) и

$$
\frac{\partial}{\partial x_{i}}\left(f_{j}-\mu_{j} g_{j}\right)= \pm 2 x_{i}-\mu_{j} \varphi^{\prime}(\|x\|) \frac{x_{i}}{\|x\|} .
$$

Если эта частная производная равна нулю и $x_{i} \neq 0$, то $\varphi^{\prime}(r)= \pm 2 r \mu_{j}^{-1}$. Согласно (15), это может случиться, только если $r=\|x\|=0$.

Теперь покажем, как выбрать параметры $\mu_{j}$, чтобы у замкнутой 1-формы $\omega^{\prime}$, заданной формулой (16), был только один особый слой. Пусть $L-$ некоторый фиксированный неособый слой слоения $\omega=0$. Поскольку $L$ является плотным в $M$ (см. п. 3.2), для всех $j=1, \ldots, m$ пересечение $L \cap U_{j}$ состоит из бесконечного 


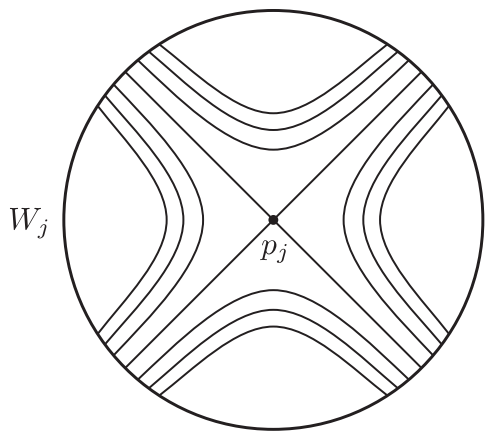

Рис. 3

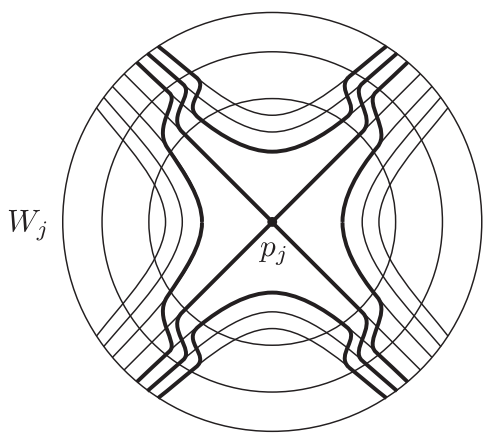

Рис. 4. Случай $\mu_{j}>0$

числа компонент связности, приближающихся к $p_{j}$ снизу и сверху. На каждой из этих компонент связности функция $f_{j}$ постоянна (ср. рис. 3$)$.

Будем говорить, что подмножество $T_{c} \subset L \cap W_{j}$ является множеством уровня, если $T_{c}=f_{j}^{-1}(c) \cap W_{j}$ для некоторого $c \in \mathbb{R}$. Заметим, что $f_{j}\left(p_{j}\right)=0$. Множество уровня $c=0$ содержит нули $p_{j}$; оно гомеоморфно конусу над произведением сфер $S^{m_{j}-1} \times S^{n-m_{j}-1}$. Любое множество уровня $T_{c}$ при $c<0$ диффеоморфно многообразию $S^{m_{j}-1} \times D^{n-m_{j}}$, а при $c>0$ - многообразию $D^{m_{j}} \times S^{n-m_{j}-1}$. Напомним, что $m_{j}-$ индекс критической точки $p_{j}$.

Пусть $\mathscr{V}_{j}=f_{j}\left(L \cap W_{j}\right) \subset \mathbb{R}-$ множество значений функции $f_{j}$ на разных множествах уровня, лежащих в слое $L$. Число нуль не принадлежит $\mathscr{V}_{j}$, поскольку по предположению слой $L$ является неособым. Однако, согласно результату, доказанному в п. 3.2 , нуль является предельной точкой $\mathscr{V}_{j}$ и, более того, замыкание одного из множеств $\mathscr{V}_{j} \cap(0, \infty), \mathscr{V}_{j} \cap(-\infty, 0)$ содержит $0 \in \mathbb{R}$.

Для модификации $\omega^{\prime}$ (заданной формулой (16)) имеем $\left.\omega^{\prime}\right|_{U_{j}}=d h_{j}$, где $h_{j}=$ $f_{j}-\mu_{j} g_{j}$. Множества уровня $T_{c}^{\prime}$ для функций $h_{j}$ определяются как $h_{j}^{-1}(c) \cap W_{j}$. Очевидно, что $T_{c}^{\prime}$ задается уравнением

$$
f_{j}(x)=\mu_{j} \varphi(\|x\|)+c, \quad x \in W_{j} .
$$

Поэтому множество уровня $T_{c}^{\prime}$ при $\|x\| \geqslant 3 / 4$ совпадает с $T_{c}$, а при $\|x\| \leqslant 1 / 2-$ с $T_{c+\mu_{j} \varepsilon}$. В кольце $1 / 2 \leqslant\|x\| \leqslant 3 / 4$ множество уровня $T_{c}^{\prime}$ гомеоморфно цилиндру.

Рис. 4 иллюстрирует различия между множествами уровня $T_{c}$ и $T_{c}^{\prime}$ при $\mu_{j}>0$.

Исследуем, как изменяется слой $L$ при замене формы $\omega$ на $\omega^{\prime}$. Здесь мы видим $L$ в топологии слоя; эта топология индуцирована на $L$ с накрытия $\widetilde{M}$ при произвольном поднятии $L \rightarrow \widetilde{M}$. Предположим сначала, что: 1$)$ индекс Морса $m_{j}$ меньше $\left.n-1 ; 2\right)$ коэффициент $\mu_{j}$ положителен; 3$)$ число $-\varepsilon \mu_{j}$ лежит в множестве $\mathscr{V}_{j}$. Тогда дополнение

$$
L-\bigcup_{\substack{c \in \mathscr{V}_{j} \\-\varepsilon \mu_{j}<c<0}} T_{c}
$$

связно и лежит в одном слое $L^{\prime}$ слоения с особенностями $\omega^{\prime}=0$. Мы видим, что слой $L^{\prime}$ получается из $L$ бесконечным количеством перестроек. А именно, 
любое множество уровня $T_{c} \subset L$, где число $c \in \mathscr{V}_{j}$ удовлетворяет неравенству $-\varepsilon \mu_{j}<c<0$, удаляется и заменяется на экземпляр ручки $D^{m_{j}} \times S^{n-m_{j}-1}$; с другой стороны, множество $T_{c} \subset L$, где $c=-\varepsilon \mu_{j}$, удаляется и заменяется конусом над произведением сфер $S^{m_{j}-1} \times S^{n-m_{j}-1}$. Следовательно, слой $L^{\prime}$ содержит критическую точку $p_{j}$.

Покажем, как можно изменить приведенную выше конструкцию в случае $m_{j}=n-1$. Поскольку $n>2$, в этом случае имеем $n-m_{j}-1<n-2$; поэтому удаление сферы $S^{n-m_{j}-1}$ не сделает слой $L$ несвязным. Мы будем предполагать, что коэффициент $\mu_{j}$ отрицателен и число $-\varepsilon \mu_{j}$ принадлежит множеству $\mathscr{V}_{j} \subset \mathbb{R}$. Дополнение

$$
L-\bigcup_{\substack{c \in \mathscr{V}_{j} \\ 0<c<-\varepsilon \mu_{j}}} T_{c}
$$

связно и лежит в одном слое $L^{\prime}$ слоения с особенностями $\omega^{\prime}=0$. Очевидно, $L^{\prime}$ получается из $L$ удалением множеств уровня $T_{c}$, где $c \in \mathscr{V}_{j}$ удовлетворяет $0<c<-\varepsilon \mu_{j}$ (каждое такое $T_{c}$ диффеоморфно $D^{m_{j}} \times S^{n-m_{j}-1}$ ), и заменой их на копии цилиндров $S^{m_{j}-1} \times D^{n-m_{j}}$. Кроме того, множество $T_{c} \subset L$, где $c=-\varepsilon \mu_{j}$, удаляется и заменяется на конус над произведением сфер $S^{m_{j}-1} \times S^{n-m_{j}-1}$.

Таким образом, мы видим, что $L^{\prime}-$ слой слоения с особенностями $\omega^{\prime}=0$, содержащий все критические точки $p_{1}, \ldots, p_{m}$.

3.4. Доказательство теоремы 3.1. Ниже будем предполагать, что выполнено неравенство $\operatorname{rk}(\xi)>1$. Случай $\operatorname{rk}(\xi)=1$ покрывается теоремой 2.1 из [15].

Результаты предыдущих пунктов позволяют завершить доказательство теоремы 3.1 в случае $n=\operatorname{dim} M>2$. Действительно, в п. 3.3 мы показали, как в произвольном классе когомологий $\xi$ выбрать морсовскую замкнутую 1-форму $\omega^{\prime}$, все нули которой лежат на одном особом слое $L^{\prime}$ слоения с особенностями $\omega^{\prime}=0$. Теперь мы можем применить технику Такенса (см. [43; c. 203-206]). А именно, мы можем найти кусочно гладкое дерево $\Gamma \subset L^{\prime}$, содержащее все нули $\omega^{\prime}$. Пусть $U \subset M$ - малая окрестность $\Gamma$, диффеоморфная $\mathbb{R}^{n}$. Можно построить непрерывное отображение $\Psi: M \rightarrow M$ со следующими свойствами:

(a) $\Psi(\Gamma)$ cостоит из одной точки $p \in \Gamma$;

(b) $\left.\Psi\right|_{M-\Gamma}$ является диффеоморфизмом на $M-\{p\}$;

(c) $\Psi(U)=U$;

(d) $\Psi$ является тождественным отображением на дополнении $\kappa$ малой окрестности $V \subset M$ дерева $\Gamma$, где замыкание $\bar{V}$ содержится в $U$.

Рассмотрим гладкую функцию $f: U \rightarrow \mathbb{R}$ такую, что $d f=\left.\omega^{\prime}\right|_{U}$; она существует и единственна с точностью до аддитивной константы. Функция $g=$ $f \circ \Psi^{-1}: U \rightarrow \mathbb{R}$ корректно определена (поскольку $\left.f\right|_{\Gamma}$ постоянна). Функция $g$ является непрерывной по универсальному свойству фактортопологии. Более того, $g$ гладкая на $M-\{p\}$. Применяя теорему 2.7 из [43], мы видим, что можно заменить $g$ гладкой функцией $h: U \rightarrow \mathbb{R}$, имеющей единственную критическую точку $p$ и такой, что $h=f$ на $U-\bar{V}$. 
Пусть $\omega^{\prime \prime}$ - замкнутая 1-форма на $M$, заданная формулами

$$
\left.\omega^{\prime \prime}\right|_{M-\bar{V}}=\left.\omega^{\prime}\right|_{M-\bar{V}} \quad \text { и }\left.\quad \omega^{\prime \prime}\right|_{U}=d h .
$$

Очевидно, что $\omega^{\prime \prime}$ - гладкая замкнутая 1-форма на многообразии $M$, не имеющая нулей на $M-\{p\}$. Более того, $\omega^{\prime \prime}$ лежит в классе когомологий $\xi=\left[\omega^{\prime}\right]$ (поскольку любая петля в $M$ гомологична петле в $M-\bar{V}$ ).

Теперь докажем теорему 3.1 в случае $n=2$. Мы должны заменить конструкцию п. 3.3 (где требовалось $n>2$ ) прямой конструкцией. Заключительное рассуждение, использующее технику Такенса, остается без изменения.

Пусть $M$ - замкнутая поверхность и $\xi \in H^{1}(M ; \mathbb{R})$ - ненулевой класс когомологий. Поверхность $M$ можно разложить в связную сумму

$$
M=M_{1} \sharp M_{2} \sharp \cdots \sharp M_{k},
$$

где каждое $M_{j}$ является тором или бутылкой Клейна, а класс когомологий $\xi_{j}=\left.\xi\right|_{M_{j}} \in H^{1}\left(M_{j} ; \mathbb{R}\right)$ ненулевой. Действительно, начнем с произвольного разложения $(\star)$ и, если $\xi$ обращается в нуль на некотором $M_{j}$, осуществим скручивание Дена вдоль вложенной окружности, представляющей собой сумму примитивного элемента $x$ из $H_{1}\left(M_{j}\right)$ и элемента $y$ из $H_{1}(M)$, на котором класс $\xi$ ненулевой. Это приводит к модифицированному разложению (19) с ненулевым $\left.\xi\right|_{M_{j}}$.

Пусть $\omega_{j}$ - замкнутая 1-форма на $M_{j}$ из класса $\xi_{j}$, не имеющая нулей; очевидно, что такая форма существует. В [13; § 9.3.2] описана конструкция связной суммы замкнутых 1-форм на поверхностях. Каждая связная "трубка" добавляет два нуля. На самом деле, есть три разных способа образования связных сумм, на рис. 9.8 из [13] они обозначены буквами А, В, С. В типе С нули лежат на одном и том же особом слое. Следовательно, используя связное суммирование типа С, получим замкнутую 1-форму $\omega$ на $M$ с $2 k-2$ нулями, которые лежат на одном особом слое слоения с особенностями $\omega=0$. Рассуждение, основанное на технике Такенса [43], применяется так же, как и при $n>2$, и в любом ненулевом классе когомологий $\xi$ приводит к замкнутой 1-форме с не более чем одним нулем.

\section{4. Замкнутые 1-формы на общих топологических пространствах}

Поскольку замкнутые 1-формы играют центральную роль в наших конструкциях, целесообразно использовать понятие замкнутых 1-форм, определенных на общих топологических пространствах. Это понятие позволит нам рассматривать более общие пространства, чем гладкие многообразия. Исчисление замкнутых 1-форм на топологических пространствах очень похоже на исчисление гладких замкнутых 1-форм на многообразиях: любую замкнутую 1-форму можно интегрировать вдоль пути; интеграл зависит только от гомотопического класса пути; любая замкнутая 1-форма представляет одномерный класс когомологий, и любая непрерывная функция $f$ определяет точную замкнутую 1-форму $d f$ - дифференциал функции $f$.

В этом разделе мы напомним основные определения; доказательства и более подробную информацию читатель может найти в книге [13]. 


\section{1. Основные определения.}

ОПРЕДЕЛЕНИЕ 4.1. Непрерывная замкнутая 1-форма $\omega$ на топологическом пространстве $X$ определяется как набор $\left\{f_{U}\right\}_{U \in \mathscr{U}}$ непрерывных вещественнозначных функций $f_{U}: U \rightarrow \mathbb{R}$, где $\mathscr{U}=\{U\}$ - открытое покрытие $X$, такой, что для любой пары $U, V \in \mathscr{U}$ разность

$$
\left.f_{U}\right|_{U \cap V}-\left.f_{V}\right|_{U \cap V}: U \cap V \rightarrow \mathbb{R}
$$

есть локально постоянная функция. Другой такой же набор $\left\{g_{V}\right\}_{V \in \mathscr{V}}$ (где $\mathscr{V}$ - некоторое другое покрытие пространства $X)$ определяет эквивалентную замкнутую 1-форму, если объединение наборов

$$
\left\{f_{U}, g_{V}\right\}_{U \in \mathscr{U}, V \in \mathscr{V}}
$$

является замкнутой 1-формой (в смысле приведенного выше определения), т. е. если для любых $U \in \mathscr{U}$ и $V \in \mathscr{V}$ функция $f_{U}-g_{V}$ локально постоянна на $U \cap V$.

Пусть $\varphi: Y \rightarrow X-$ непрерывное отображение и $\omega$ - непрерывная замкнутая 1 -форма на пространстве $X$. Тогда индуцированная замкнутая 1 -форма $\varphi^{*} \omega$ определяется следующим образом. Пусть $\omega=\left\{f_{U}\right\}_{U \in \mathscr{U}}$, где $\mathscr{U}$ - открытое покрытие $X$. Семейство $\varphi^{-1} \mathscr{U}=\left\{\varphi^{-1}(U)\right\}_{U \in \mathscr{U}}$ является открытым покрытием пространства $Y$, и функции $f_{U} \circ \varphi$ определяют непрерывную замкнутую 1-форму относительно покрытия $\varphi^{-1} \mathscr{U}$.

В качестве примера рассмотрим открытое покрытие $\mathscr{U}=\{X\}$, состоящее из всего пространства $X$. Тогда любая непрерывная функция $f: X \rightarrow \mathbb{R}$ определяет замкнутую 1-форму на $X$, которую обозначим через $d f$. Для двух непрерывных функций $f, g: X \rightarrow \mathbb{R}$ равенство $d f=d g$ имеет место тогда и только тогда, когда разность $f-g: X \rightarrow \mathbb{R}$ является локально постоянной функцией.

4.2. Интегрирование. Непрерывные замкнутые 1-формы можно интегрировать вдоль непрерывных путей. Пусть $\omega$ - непрерывная замкнутая 1-форма на $X$, заданная набором непрерывных вещественнозначных функций $\left\{f_{U}\right\}_{U \in \mathscr{U}}$ относительно открытого покрытия $\mathscr{U}$ пространства $X$. Пусть $\gamma$ : $[0,1] \rightarrow X-$ непрерывный путь. Интеграл $\int_{\gamma} \omega$ определяется следующим образом. Найдем такое разбиение $t_{0}=0<t_{1}<\cdots<t_{N}=1$ отрезка $[0,1]$, для которого при любом $i$ образ $\gamma\left[t_{i}, t_{i+1}\right]$ целиком содержится в некотором открытом множестве $U_{i} \in \mathscr{U}$. Тогда мы определяем

$$
\int_{\gamma} \omega=\sum_{i=0}^{N-1}\left[f_{U_{i}}\left(\gamma\left(t_{i+1}\right)\right)-f_{U_{i}}\left(\gamma\left(t_{i}\right)\right)\right] .
$$

Стандартные рассуждения показывают, что интеграл (19) не зависит от выбора разбиения отрезка и открытого покрытия $\mathscr{U}$.

Для любой пары непрерывных путей $\gamma, \gamma^{\prime}:[0,1] \rightarrow X$ с общими начальной $\gamma(0)=\gamma^{\prime}(0)$ и конечной $\gamma(1)=\gamma^{\prime}(1)$ точками имеет место равенство

$$
\int_{\gamma} \omega=\int_{\gamma^{\prime}} \omega
$$

при условии, что $\gamma$ и $\gamma^{\prime}$ гомотопны относительно границы. 
4.3. Когомологический класс замкнутой 1-формы. Любая непрерывная замкнутая 1-форма $\omega$ определяет гомоморфизм периодов

$$
\pi_{1}\left(X, x_{0}\right) \rightarrow \mathbb{R}, \quad[\gamma] \mapsto \int_{\gamma} \omega \in \mathbb{R},
$$

задаваемый интегрированием вдоль петель $\gamma:[0,1] \rightarrow X$ с $\gamma(0)=x_{0}=\gamma(1)$. Образом этого гомоморфизма является подгруппа $\mathbb{R}$, ранг которой называется рангом (в отечественной литературе - степенью иррациональности. - Прим. перев.) 1-формы $\omega$ и обозначается $\operatorname{rk}(\omega)$.

Напомним, что топологическое пространство $X$ называется гомологически локально $n$-связным, если для каждой точки $x \in X$ и любой окрестности $U$ точки $x$ существует такая окрестность $V$ точки $x$, что $V \subset U$ и индуцированный гомоморфизм приведенных целочисленных сингулярных гомологий $\widetilde{H}_{q}(V) \rightarrow$ $\widetilde{H}_{q}(U)$ является тривиальным для всех $q \leqslant n$. Топологическое пространство $X$ называется локально линейно связным тогда и только тогда, когда оно гомологически локально 0-связно.

Лемма 4.2. Пусть $X$ - локально линейно связное топологическое пространство. Непрерывная замкнутая 1-форма $\omega$ на $X$ равна $d f$ для некоторой непреръвной функции $f: X \rightarrow \mathbb{R}$ тогда и только тогда, когда при любом въборе отмеченной точки $x_{0} \in X$ гомоморфизм периодов (20), определенный формой $\omega$, является тривиальным.

ДокАЗАтЕльСтво. Если $\omega=d f$, то равенство $\int_{\gamma} \omega=f(q)-f(p)$ имеет место для любого пути $\gamma$ в $X$, где $q=\gamma(1), p=\gamma(0)$. Следовательно, $\int_{\gamma} \omega=0$, если $\gamma$
является петлей.

В обратную сторону: предположим, что гомоморфизм периодов (20) тривиален. Из нашего предположения о пространстве $X$ следует, что компоненты связности $X$ являются открытыми и линейно связными. В каждой компоненте связности $X$ отметим точки $x_{i} \in X$. Можно определить непрерывную функцию $f: X \rightarrow \mathbb{R}$ формулой

$$
f(x)=\int_{x_{i}}^{x} \omega,
$$

где $x$ и $x_{i}$ принадлежат одной и той же компоненте связности пространства $X$ и интегрирование производится вдоль произвольного пути, соединяющего $x_{i}$ c $x$. Результат интегрирования не зависит от выбора пути, поскольку по нашему предположению гомоморфизм периодов (20) является тривиальным. Предположим, что $\omega$ задана набором непрерывных функции $f_{U}: U \rightarrow \mathbb{R}$ относительно открытого покрытия $\{U\}$ пространства $X$. Тогда для любых двух точек $x, y$, принадлежащих одной и той же компоненте связности $U$, имеем

$$
f(y)-f(x)=\int_{x}^{y} \omega=f_{U}(y)-f_{U}(x),
$$

где интеграл принимается как интеграл вдоль любого непрерывного пути, соединяющего $x$ и $y$. Это показывает, что функция $f-f_{U}$ является локально постоянной на $U$. Следовательно, $d f=\omega$. Лемма 4.2 доказана. 
Любая непрерывная замкнутая 1-форма $\omega$ на топологическом пространстве $X$ определяет класс сингулярных когомологий $[\omega] \in H^{1}(X ; \mathbb{R})$. Он определяется гомоморфизмом периодов $(20)$, если рассматривать этот гомоморфизм как элемент пространства $\operatorname{Hom}\left(H_{1}(X) ; \mathbb{R}\right)=H^{1}(X ; \mathbb{R})$. Как следует из приведенной выше леммы, две непрерывные замкнутые 1-формы $\omega$ и $\omega^{\prime}$ на пространстве $X$ лежат в одном и том же классе когомологий $[\omega]=\left[\omega^{\prime}\right]$ тогда и только тогда, когда их разность $\omega-\omega^{\prime}$ равна $d f$, где $f: X \rightarrow \mathbb{R}$ - непрерьвная функиия. Здесь мы предполагаем, что $X$ - локально линейно связное пространство.

Лемма 4.3. Пусть $X$ - паракомпактное хаусдорфово гомологически локально односвязное топологическое пространство. Тогда любой класс сингулярных когомологий $\xi \in H^{1}(X ; \mathbb{R})$ можно представить непрерьвной замкнутой 1-формой на $X$.

\section{1-формы Ляпунова для потоков}

В работах [21], [22] Конли показал, что любой непрерывный поток $\Phi: X \times$ $\mathbb{R} \rightarrow X$ на компактном метрическом пространстве $X$ "разлагается" на цепнорекуррентный и градиентоподобный поток. Более точно, он доказал существование такой непрерывной функции $L: X \rightarrow \mathbb{R}$, которая:

(i) убывает вдоль любой орбиты потока в дополнении $X-R$ к цепно-рекуррентному множеству $R \subset X$ функции $\Phi$ и

(ii) постоянна на компонентах связности $R$.

Такая функция $L$ называется функцией Ляпунова для $\Phi$.

Результат Конли о существовании играет фундаментальную роль в его программе понимания общих потоков как набора изолированных инвариантных множеств, связанных гетероклиническими орбитами.

Более общее понятие 1-формы Ляпунова было введено в работе [16]. Ляпуновские 1-формы позволяют продвинуться на один шаг дальше по сравнению с ляпуновскими функциями и анализировать также поток внутри цепно-рекуррентного множества $R$. Ляпуновские 1-формы дают важный инструмент для применения методов теории гомотопий к динамическим системам.

Проблема существования непрерывных ляпуновских 1-форм впервые рассматривалась в работе [17] в применении к компактным метрическим пространствам, непрерывным потокам и непрерывным замкнутым 1-формам. В этом разделе мы представим результат работы [18] касательно гладкой версии этой проблемы: мы будем интересоваться построением гладких ляпуновских 1-форм для гладких потоков на гладких многообразиях. Эти условия формулируются в терминах гомологических свойств потока; в частности, мы используем асимптотические циклы Шварцмана $\mathscr{A}_{\mu}(\Phi) \in H_{1}(M ; \mathbb{R})$ для потоков.

Мы хотели бы порекомендовать читателю работы Ланцева [19], [20].

Пусть $V$ - гладкое векторное поле на гладком многообразии $M$. Предположим, что $V$ задает непрерывный поток $\Phi: M \times \mathbb{R} \rightarrow M$ и $Y \subset M$ - замкнутое инвариантное относительно потока подмножество.

ОПРЕДЕЛЕНИЕ 5.1. Гладкая замкнутая 1-форма $\omega$ на многообразии $M$ называется 1-формой Ляпунова пары $(\Phi, Y)$, если она имеет следующие свойства: $(\Lambda 1)$ функция $\iota_{V}(\omega)=\omega(V)$ отрицательна на $M-Y$; 
$\left.(\Lambda 2) \omega\right|_{Y}=0$ и существует гладкая функция $f: U \rightarrow \mathbb{R}$, определенная на открытой окрестности $U$ множества $Y$, такая, что $\left.\omega\right|_{U}=d f$.

Это определение является модификацией понятия ляпуновской 1-формы, введенного в [16; §6]. Определение 5.1 можно также сопоставить с определением ляпуновской 1-формы в непрерывной ситуации, введенным в работе [17]. Приведенное выше условие $(\Lambda 1)$, очевидно, несколько сильнее, чем условие (L1) из определения 1 в [17]. Условие $(\Lambda 2)$ похоже на (L2) из определения 1 в [17], но они не эквивалентны.

Имеются различные естественные альтернативы условию $(\Lambda 2)$. Приведем одно из них:

$\left(\Lambda 2^{\prime}\right)$ 1-форма $\omega$, рассматриваемая как отображение $\omega: M \rightarrow T^{*}(M)$, обращается в нуль на $Y$.

Очевидно, что из $(\Lambda 2)$ следует $\left(\Lambda 2^{\prime}\right)$. Можно доказать, что при некоторых дополнительных предположениях верно и обратное:

Лемма 5.2. Если класс когомологий де Рама $\xi$, заданный 1-формой $\omega$, является иелочисленным, т.е. $\xi=[\omega] \in H^{1}(M ; \mathbb{Z})$, то условия $\left(\Lambda 2^{\prime}\right)$ и $(\Lambda 2)$ эквивалентны.

ДокАЗАтельство. Очевидно, нам надо только доказать, что из $\left(\Lambda 2^{\prime}\right)$ следует $(\Lambda 2)$. Поскольку $\xi$ - целочисленный класс, существует такое гладкое отображение $\varphi: M \rightarrow S^{1}$, что $\omega=\varphi^{*}(d \theta)$, где $d \theta$ - стандартная угловая 1-форма на окружности $S^{1}$. Пусть $\alpha \in S^{1}$ - регулярное значение $\varphi$. Предположим, что $\left(\Lambda 2^{\prime}\right)$ выполнено, тогда $U=M-\varphi^{-1}(\alpha)$ - открытая окрестность $Y$. Ясно, что $\left.\omega\right|_{U}=d f$, где $f: U \rightarrow \mathbb{R}$ - гладкая функция, связанная с $\varphi$ формулой $\varphi(x)=\exp (i f(x))$ для любого $x \in U$. Следовательно, условие $(\Lambda 2)$ выполнено. Лемма доказана.

Лемма 5.3. Условия $\left(\Lambda 2^{\prime}\right)$ и $(\Lambda 2)$ эквивалентны, если $Y$ является евклидовым окрестностным ретрактом $(E N R)$.

ДоказАтельство. Как и прежде, нам надо показать, что $\left(\Lambda 2^{\prime}\right) \Rightarrow(\Lambda 2)$. Так как $Y$ является ENR, оно допускает такую открытую окрестность $U \subset M$, что вложение $i_{U}: U \rightarrow M$ гомотопно $i_{Y} \circ r$, где $i_{Y}: Y \rightarrow M-$ вложение и $r: U \rightarrow Y-$ ретракция (см. [44; гл. $4, \S 8$, следствие 8.7]). Отметим точку $x_{j}$ в каждой компоненте связности $U_{j}$ окрестности $U$ и определим гладкую функцию $f_{j}: U_{j} \rightarrow \mathbb{R}$ формулой

$$
f_{j}(x)=\int_{x_{j}}^{x} \omega, \quad x \in U_{j} .
$$

Последний интеграл не зависит от выбора пути интегрирования в $U_{j}$, соединяющего точку $x_{j}$ с $x$. Это утверждение эквивалентно обнулению интеграла $\int_{\gamma} \omega$ для любой петли $\gamma$, лежащей в $U$. Чтобы проверить это, мы применим ретракцию и убедимся в гомотопности $\gamma$ петле $\gamma_{1}=r \circ \gamma$, лежащей в $Y$; получаем, что $\int_{\gamma} \omega=\int_{\gamma_{1}} \omega=0$ в силу $\left(\Lambda 2^{\prime}\right)$. Очевидно, что функции $f_{j}$ в совокупности определяют гладкую функцию $f: U \rightarrow \mathbb{R}$ такую, что $d f=\left.\omega\right|_{U}$. Лемма 5.3 доказана. 
Интересный класс примеров ляпуновских 1-форм можно получить следующим образом. Пусть $\omega$ - гладкая замкнутая 1-форма на замкнутом римановом многообразии $M$. Рассмотрим противоположное к градиенту векторное поле $V$ 1-формы $\omega$, т. е. $\langle V, X\rangle=-\omega(X)$ для любого векторного поля $X$ на $M$, где $\langle\cdot, \cdot\rangle$ - риманова метрика. Обозначим через $\Phi$ поток, индуцированный векторным полем $V$, и пусть $Y$ - множество нулей 1-формы $\omega$. Очевидно, что условия $(\Lambda 2)$ и $\left(\Lambda 2^{\prime}\right)$ выполнены. Если либо когомологический класс 1 -формы $\omega$ целочисленный, либо $Y$ является ENR, то (по двум леммам, приведенным выше) $\omega$ является 1-формой Ляпунова для пары $(\Phi, Y)$.

Основной целью данного раздела является нахождение топологических условий, гарантирующих существование 1-формы Ляпунова $\omega$ для заданного векторного поля $V$ на $M$ и из фиксированного класса когомологий $\xi \in H^{1}(M ; \mathbb{R})$.

Асимптотические циклы Шварцмана. Пусть $M$ - замкнутое гладкое многообразие и $V$ - гладкое векторное поле. Пусть $\Phi: M \times \mathbb{R} \rightarrow M-$ поток, порожденный полем $V$. Рассмотрим инвариантную относительно $\Phi$ борелевскую меру $\mu$ на $M$. Согласно [23], эти данные определяют вещественный гомологический класс

$$
\mathscr{A}_{\mu}=\mathscr{A}_{\mu}(\Phi) \in H_{1}(M ; \mathbb{R}),
$$

называемый асимптотическим ииклом потока $\Phi$, соответствующим мере $\mu$. Класс $\mathscr{A}_{\mu}$ определяется следующим образом. Значение класса когомологий де Рама $\xi \in H^{1}(M ; \mathbb{R})$ на искомом цикле $\left\langle\xi, \mathscr{A}_{\mu}\right\rangle \in \mathbb{R}$ задается интегралом

$$
\left\langle\xi, \mathscr{A}_{\mu}\right\rangle=\int_{M} \iota_{V}(\omega) d \mu
$$

где $\omega$ - замкнутая 1-форма из класса $\xi$. Заметим, что значение $\left\langle\xi, \mathscr{A}_{\mu}\right\rangle$ корректно определено, т. е. зависит только от класса когомологий $\xi$ формы $\omega$ (см. [23; c. 277]). Действительно, при замене $\omega$ на $\omega^{\prime}=\omega+d f$, где $f: M \rightarrow \mathbb{R}$ - гладкая функция, интеграл (21) меняется на следующую величину:

$$
\int_{M} V(f) d \mu=\lim _{s \rightarrow 0} \frac{1}{s} \int_{M}\{f(x \cdot s)-f(x)\} d \mu(x) .
$$

Здесь $V(f)$ обозначает производную функции $f$ вдоль векторного поля $V$, а $x \cdot s$ - поток $\Phi(x, s)$, порожденный векторным полем $V$. Поскольку мера $\mu$ инвариантна относительно потока, то интеграл в правой части (22) равен нулю для всех $f$. Очевидно, что правая часть формулы (21) является линейной функцией от $\xi \in H^{1}(M ; \mathbb{R})$. Поэтому существует единственный вещественный класс гомологий $\mathscr{A}_{\mu} \in H_{1}(M ; \mathbb{R})$, который удовлетворяет условию $(21)$ для всех $\xi \in H^{1}(M ; \mathbb{R})$.

Необходимые условия. Зафиксируем поток $\Phi$ и будем менять инвариантные меры $\mu$. Так как класс $\mathscr{A}_{\mu} \in H_{1}(M ; \mathbb{R})$ линейно зависит от $\mu$, то множество асимптотических циклов $\mathscr{A}_{\mu}$, соответствующих всем $\Phi$-инвариантным положительным мерам $\mu$, образует выпуклый конус в векторном пространстве $H_{1}(M ; \mathbb{R})$. 
ПреДЛОЖЕНИЕ 5.4. Пусть существует 1-форма Ляпунова для $(\Phi, Y)$, лежащая в классе когомологий $\xi \in H^{1}(M ; \mathbb{R})$. Тогда для любой инвариантной относительно $\Phi$ положительной борелевской меры $\mu$ на $M$

$$
\left\langle\xi, \mathscr{A}_{\mu}\right\rangle \leqslant 0
$$

равенство в (23) имеет место тогда и только тогда, когда дополнение $к Y$ имеет меру нуль. Более того, ограничение класса $\xi$ на $Y$, рассматриваемое как класс когомологий Чеха,

$$
\left.\xi\right|_{Y} \in \check{H}^{1}(Y ; \mathbb{R})
$$

равно нулю: $\left.\xi\right|_{Y}=0$.

Доказательство. Пусть $\omega$ есть 1-форма Ляпунова для $(\Phi, Y)$ из когомологического класса $\xi$. Согласно определению 5.1 , функция $\iota_{V}(\omega)$ отрицательна на $M-Y$ и равна нулю на $Y$. Получаем, что интеграл

$$
\int_{M} \iota_{V}(\omega) d \mu=\left\langle\xi, \mathscr{A}_{\mu}\right\rangle
$$

является неположительным.

Предположив, что $\mu(M-Y)>0$, найдем такой компакт $K \subset M-Y$, что $\mu(K)>0$; это следует из теоремы Рисса (см., например., [45; теорема 2.3(iv), c. 256]). Существует константа $\varepsilon>0$ такая, что $\left.\iota_{V}(\omega)\right|_{K} \leqslant-\varepsilon$. Следовательно,

$$
\int_{M} \iota_{V}(\omega) d \mu \leqslant-\varepsilon \mu(K)<0
$$

Поэтому значение $\left\langle\xi, \mathscr{A}_{\mu}\right\rangle$ является строго отрицательным, если носитель меры $\mu$ не сосредоточен в $Y$.

Для доказательства второго утверждения заметим (см. [46]), что когомологии Чеха $\check{H}^{1}(Y ; \mathbb{R})$ совпадают с прямым пределом сингулярных когомологий:

$$
\check{H}^{1}(Y ; \mathbb{R})=\lim _{W \supset Y} H^{1}(W ; \mathbb{R}),
$$

где $W$ пробегает все открытые окрестности множества $Y$. Очевидно, что ввиду $(\Lambda 2)$ выполнено $\left.\xi\right|_{U}=0 \in H^{1}(U ; \mathbb{R})$ (согласно теореме де Рама). Отсюда следует утверждение. Предложение 5.4 доказано.

Цепно-рекуррентное множество $R_{\xi}$. Нашей целью является построение 1-формы Ляпунова $\omega$ из заданного класса когомологий $\xi \in H^{1}(M ; \mathbb{R})$ для пары $(\Phi, Y)$ при заданном потоке $\Phi$. Естественным кандидатом на роль $Y$ является подмножество $R_{\xi}=R_{\xi}(\Phi)$ цепно-рекуррентного множества $R=R(\Phi)$, которое определено в [17]. Напомним кратко определение.

Зададим на $M$ риманову метрику и обозначим через $d$ соответствующую функцию расстояния. Если заданы $\delta>0, T>1$, то $(\delta, T)$-цепъю из $x \in M$ в $y \in M$ называются конечная последовательность $x_{0}=x, x_{1}, \ldots, x_{N}=y$ точек многообразия $M$ и числа $t_{1}, \ldots, t_{N} \in \mathbb{R}$ такие, что $t_{i} \geqslant T$ и $d\left(x_{i-1}\right.$. $\left.t_{i}, x_{i}\right)<\delta$ для любых $1 \leqslant i \leqslant N$. Здесь мы используем обозначение $\Phi(x, t)=$ $x \cdot t$. Цепно-рекуррентное множество $R=R(\Phi)$ потока $\Phi$ определяется как 
множество всех таких точек $x \in M$, для которых при любых $\delta>0$ и $T>1$ существует $(\delta, T)$-цепь с началом и концом в точке $x$. Цепно-рекуррентное множество $R$ является замкнутым и инвариантным относительно потока.

Для данного когомологическиго класса $\xi \in H^{1}(M ; \mathbb{R})$ существует естественное накрытие $p_{\xi}: \widetilde{M}_{\xi} \rightarrow M$, ассоциированное с $\xi$. Петля $\gamma:[0,1] \rightarrow M$ поднимается до петли в $\widetilde{M}_{\xi}$, если и только если значение когомологического класса $\xi$ на гомологическом классе $[\gamma] \in H_{1}(M ; \mathbb{Z})$ равно нулю: $\langle\xi,[\gamma]\rangle=0$.

Поток $\Phi$ однозначно поднимается до потока $\widetilde{\Phi}$ на накрывающей $\widetilde{M}_{\xi}$. Рассмотрим цепно-рекуррентное множество $R(\widetilde{\Phi}) \subset \widetilde{M}_{\xi}$ поднятого потока и обозначим через $R_{\xi}=p_{\xi}(R(\widetilde{\Phi})) \subset M$ его проекцию на $M$. Множество $R_{\xi}$ называется цепно-рекуррентным множеством, ассоциированным с классом когомологий $\xi$. Очевидно, что $R_{\xi}$ является замкнутым, инвариантным относительно $\Phi$ и $R_{\xi} \subset R$.

Обозначим через $C_{\xi}$ дополнение к множеству $R_{\xi}$ в $R$ :

$$
C_{\xi}=R-R_{\xi}
$$

Приведем пример, иллюстрирующий определение $R_{\xi}$. Рассмотрим гладкий поток на замкнутом многообразии $M$, цепно-рекуррентное множество $R$ которого состоит из конечного числа неподвижных точек и периодических орбит. Для заданного класса когомологий $\xi \in H^{1}(M ; \mathbb{R})$ цепно-рекуррентное множестве $R_{\xi}$ является объединением всех неподвижных точек и таких периодических орбит, гомологические классы $z \in H_{1}(M ; \mathbb{Z})$ которых удовлетворяют равенству $\langle\xi, z\rangle=0$.

В общем случае все неподвижные точки потока принадлежат множеству $R_{\xi}$. Точки периодической орбиты принадлежат $R_{\xi}$, если для гомологического класса $z \in H_{1}(X ; \mathbb{Z})$ этой орбиты выполнено равенство $\langle\xi, z\rangle=0$.

Может случиться, что точки периодической орбиты принадлежат множеству $R_{\xi}$, даже если $\langle\xi, z\rangle \neq 0$ для гомологического класса $z$ орбиты. Такую возможность иллюстрирует следующий пример (см. [17]).

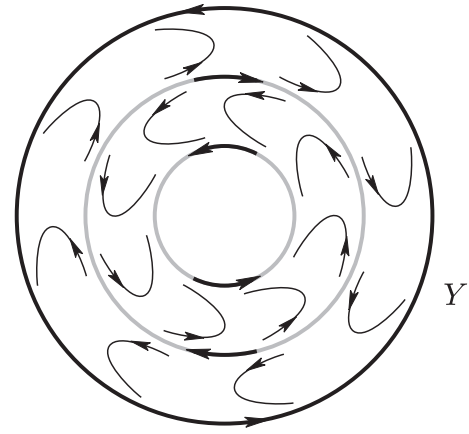

Рис. 5. Поток на плоском кольце $Y$

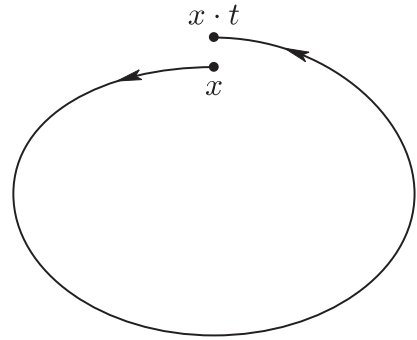

Рис. 6. $(\delta, T)$-цикл

Рассмотрим поток на плоском кольце $Y \subset \mathbb{C}$, показанный на рис. 5 . В полярных координатах $(r, \varphi)$ кольцо $Y$ задается неравенствами $1 \leqslant r \leqslant 3$, а сам 
поток - системой дифференциальных уравнений

$$
\dot{r}=(r-1)^{2}(r-3)^{2}(r-5)^{2}, \quad \dot{\varphi}=\sin \left(r \cdot \frac{\pi}{2}\right) .
$$

Для $k=1,2,3$ через $C_{k}$ обозначим окружность $r=2 k-1$. Окружности $C_{1}$, $C_{2}, C_{3}$ инвариантны относительно потока. Движение вдоль окружностей $C_{1}$ и $C_{3}$ имеет постоянную угловую скорость, равную единице. Отождествляя точки $(r, \varphi) \in C_{1}$ с точками $(5 r, \varphi) \in C_{3}$, получим тор $X=Y / \simeq$ и поток $\Phi: X \times \mathbb{R} \rightarrow X$ на нем. Образы окружностей $C_{1}, C_{2}, C_{3} \subset Y$ задают две окружности $C_{1}^{\prime}=C_{3}^{\prime}$ и $C_{2}^{\prime}$ на торе $X$. Пусть $\xi \in H^{1}(X ; \mathbb{R})$ - ненулевой класс когомологий, который является обратным образом класса когомологий кольца $Y$. Можно проверить, что в этом примере множество $R_{\xi}(\Phi)$ совпадает со всем тором $X$. В частности, $R_{\xi}(\Phi)$ содержит периодические орбиты $C_{1}^{\prime}=C_{3}^{\prime}$ и $C_{2}^{\prime}$, хотя очевидно, что $\left\langle\xi,\left[C_{k}^{\prime}\right]\right\rangle \neq 0$.

Другое определение $R_{\xi}$, не использующее накрывающее пространство $\widetilde{M}_{\xi}$, можно найти в [17].

Для формулировки основного результата этого раздела нам понадобится следующее определение. $(\delta, T)$-цикл потока $\Phi$ определяется как пара $(x, t)$, где $x \in M$ и $t>T$, такая, что $d(x, x \cdot t)<\delta$ (см. рис. 6). Если $\delta$ достаточно мало, то $(\delta, T)$-цикл однозначно и канонически определяет гомологический класс $z \in H_{1}(M ; \mathbb{Z})$, который представляется траекторией потока из точки $x$ в точку $x \cdot t$, продолженной "короткой” дугой, соединяющей $x \cdot t$ с $x$. См. [17] для более подробного ознакомления.

Теорема 5.5 [18]. Пусть $V$ - гладкое векторное поле на гладком замкнутом многообразии $M$. Обозначим $\Phi: M \times \mathbb{R} \rightarrow M$ поток, порожденный $V$. Пусть $\xi \in H^{1}(M ; \mathbb{R})$ - такой когомологический класс, что ограничение $\left.\xi\right|_{R_{\xi}}$, рассматриваемое как класс когомологий Чеха $\left.\xi\right|_{R_{\xi}} \in \check{H}^{1}\left(R_{\xi} ; \mathbb{R}\right)$, равно нулю. Тогда следующие условия на $\xi$ эквивалентны.

(I) В классе когомологий $\xi$ существует гладкая 1-форма Ляпунова для пары $\left(\Phi, R_{\xi}\right)$, и подмножество $C_{\xi}$ замкнуто.

(II) Для любой римановой метрики на $M$ существуют $\delta>0 u T>1$, для которых класс когомологий $z \in H_{1}(M ; \mathbb{Z})$, ассоциированный $c(\delta, T)$-ииклом $(x, t)$ этого потока, где $x \in C_{\xi}$, удовлетворяет условию $\langle\xi, z\rangle \leqslant-1$.

(III) Подмножество $C_{\xi}$ замкнуто, и существует такая константа $\eta>0$, что для любой инвариантной относительно $\Phi$ положительной борелевской меры н на $M$ асимптотический цикл $\mathscr{A}_{\mu}=\mathscr{A}_{\mu}(\Phi) \in H_{1}(M ; \mathbb{R})$ удовлетворяет неравенству

$$
\left\langle\xi, \mathscr{A}_{\mu}\right\rangle \leqslant-\eta \cdot \mu\left(C_{\xi}\right) .
$$

(IV) Подмножество $C_{\xi}$ замкнуто, и для любой инвариантной относительно $\Phi$ положительной борелевской меры $\mu$ на $X$ с $\mu\left(C_{\xi}\right)>0$ асимптотический иикл $\mathscr{A}_{\mu}=\mathscr{A}_{\mu}(\Phi) \in H_{1}(M ; \mathbb{R})$ удовлетворяет неравенству

$$
\left\langle\xi, \mathscr{A}_{\mu}\right\rangle<0
$$

Условие (24) можно переформулировать с использованием понятия квазирегулярной точки. Напомним, что $x \in X$ называется квазирегулярной точкой 
потока $\Phi: X \times \mathbb{R} \rightarrow X$, если для любой непрерывной функции $f: X \rightarrow \mathbb{R}$ существует предел

$$
\lim _{t \rightarrow \infty} \frac{1}{t} \int_{0}^{t} f(x \cdot s) d s .
$$

Как следует из эргодической теоремы, подмножество $Q \subset X$ всех квазирегулярных точек имеет полную меру относительно любой $\Phi$-инвариантной положительной борелевской меры на $X$. Из теоремы Рисса о представлении (см., например, [45; теорема 2.3(iv)]) можно заключить, что для любой квазирегулярной точки $x \in Q$ существует единственная положительная, инвариантная относительно потока мера Бореля $\mu_{x}$ с $\mu_{x}(X)=1$ такая, что

$$
\lim _{t \rightarrow \infty} \frac{1}{t} \int_{0}^{t} f(x \cdot s) d s=\int_{X} f d \mu_{x}
$$

для любой непрерывной функции $f$. Ниже мы используем хорошо известный факт, что положительная $\Phi$-инвариантная мера Бореля $\mu$ с $\mu(X)=1$ принадлежит слабому * замыканию выпуклой оболочки множества мер $\mu_{x}, x \in Q$.

Если подмножество $C_{\xi} \subset X$ замкнуто (и, следовательно, компактно), можно применить приведенные выше факты к ограничению потока на $C_{\xi}$. Пусть $\omega-$ произвольная гладкая замкнутая 1-форма из класса когомологий $\xi$. Для любой квазирегулярной точки $x \in C_{\xi}$ потока $\left.\Phi\right|_{C_{\xi}}$ имеем

$$
\begin{aligned}
\lim _{t \rightarrow \infty} \frac{1}{t} \int_{x}^{x \cdot t} \omega & =\lim _{t \rightarrow \infty} \frac{1}{t} \int_{0}^{t} \iota_{V}(\omega)(x \cdot s) d s \\
& =\int_{M} \iota_{V}(\omega) d \mu_{x}=\left\langle\xi, \mathscr{A}_{\mu_{x}}\right\rangle .
\end{aligned}
$$

Следовательно, можно заключить, что условие (III) эквивалентно следующему.

(III') Множество $C_{\xi}$ является замкнутым, и существует такая постоянная $\eta>0$, что для любой квазирегулярной точки $x \in C_{\xi}$ выполнено

$$
\lim _{t \rightarrow \infty} \frac{1}{t} \int_{x}^{x \cdot t} \omega \leqslant-\eta,
$$

где $\omega$ - произвольная замкнутая 1-форма из класса $\xi$.

Значение предела (29) не зависит от выбора замкнутой 1-формы $\omega$; единственное, что нужно потребовать, - чтобы $\omega$ принадлежала классу когомологий $\xi$.

В частном случае $\xi=0$ множество $C_{\xi}$ пусто и $R=R_{\xi}$. Приведенное выше утверждение тогда сводится к следующей хорошо известной теореме Конли (см. [21] и [47; теорема 3.14]).

Теорема 5.6 (Конли). Пусть $V$ - гладкое векторное поле на гладком замкнутом многообразии $M$. Обозначим через $\Phi: M \times \mathbb{R} \rightarrow M$ поток, порожденный $V$, а через $R$ - цепно-рекуррентное множество потока $\Phi$. Тогда для пары $(\Phi, R)$ существует гладкая функиия Ляпунова $L: M \rightarrow \mathbb{R}$. Это означает, что $V(L)<0$ на $M-R$ u $d L=0$ поточечно на $R$. 
Доказательство теоремы 5.5 можно найти в [18].

Укажем также на работу [17], в которой изучались непрерывные 1-формы Ляпунова. Статья [17] содержит также сравнение результатов о существования 1-форм Ляпунова с теоремами Фрида [48] о существовании перекрестных сечений потоков.

Наконец, опишем один класс потоков, для которых существует когомологический класс $\xi$, удовлетворяющий всем условиям теоремы 5.5.

Пусть $M$ - замкнутое гладкое многообразие с гладким векторным полем $v$ на нем. Пусть $\Psi: M \times \mathbb{R} \rightarrow M-$ поток поля $v$. Предположим, что цепнорекуррентное множество $R(\Psi)$ состоит из двух непересекающихся замкнутых множеств: $R(\Psi)=R_{1} \cup R_{2}$, где $R_{1} \cap R_{2}=\varnothing$. По этим данным построим такой поток $\Phi$ на

$$
X=M \times S^{1},
$$

что $R_{\xi}(\Phi)=R_{1} \times S^{0}, C_{\xi}=R_{2} \times S^{1}$. Здесь $\xi \in H^{1}(X ; \mathbb{Z})$ обозначает класс когомологий де Рама 1 -формы $-d \theta$, где $\theta \in[0,2 \pi]$ - угловая координата на окружности $S^{1} ; S^{0} \subset S^{1}$ является двухточечным множеством.

Нам понадобятся два векторных поля $w_{1}$ и $w_{2}$ на окружности $S^{1}, w_{1}=$ $\cos (\theta) \cdot \frac{\partial}{\partial \theta}$ и $w_{2}=\frac{\partial}{\partial \theta}$. Поле $w_{1}$ имеет два нуля $\left\{p_{1}, p_{2}\right\}=S^{0} \subset S^{1}$, соответствующие углам $\theta=\pi / 2$ и $\theta=3 \pi / 2$.

Пусть $f_{i}: M \rightarrow[0,1]$, где $i=1,2,-$ две гладкие функции с непересекающимися носителями, удовлетворяющие условиям $\left.f_{1}\right|_{R_{1}}=1,\left.f_{2}\right|_{R_{2}}=1$.

Рассмотрим поток $\Phi: X \times \mathbb{R} \rightarrow X$, заданный векторным полем

$$
V=v+f_{1} w_{1}+f_{2} w_{2}
$$

Любая траектория поля $V$ имеет вид $(\gamma(t), \theta(t))$, где $\dot{\gamma}(t)=v(\gamma(t))$, т. е. $\gamma(t)-$ траектория поля $v$. Отсюда следует, что цепно-рекуррентное множество поля $V$ содержится в $R(\Psi) \times S^{1}$. Над $R_{1}$ имеем вертикальное векторное поле $w_{1}$ вдоль окружности с двухточечным цепно-рекуррентным множеством $S^{0} \subset S^{1}$. Над $R_{2}$ имеем вертикальное векторное поле $w_{2}$, цепно-рекуррентное множество которого есть вся окружность $S^{1}$. Мы видим, что $R_{1} \times S^{0}=R_{\xi}(\Phi)$, $R_{2} \times S^{1}=C_{\xi}$. Следовательно,

$$
\left.\xi\right|_{R_{\xi}}=0
$$

и множество $C_{\xi}$ замкнуто. Несложно проверить, что условие (III) теоремы 5.5 , как и другие условия этой теоремы, выполнены.

Другие примеры можно найти в [17; §7].

Подход к изучению динамических систем, в котором вместо замкнутых 1-форм Ляпунова используются коциклы, предложен в [49], [50].

\section{6. Понятие категории относительно когомологического класса}

В этом разделе мы опишем различные обобщения классического понятия категории Люстерника-Шнирельмана, которые отражают интересные динамические свойства потоков на многообразиях. Эти новые понятия зависят от выбора когомологического класса $\xi \in H^{1}(X ; \mathbb{R})$, и в случае $\xi=0$ все они равны классическому инварианту $\operatorname{cat}(X)$. 
6.1. Подвижные подмножества. Вначале введем понятие подвижности подмножества относительно замкнутой 1-формы.

ОПРЕДЕЛЕНИЕ 6.1. Пусть $\omega$ - замкнутая 1-форма на топологическом пространстве $X$. Подмножество $A \subset X$ называется $N$-подвижным относительно $\omega$ (где $N$ - целое число), если существует гомотопия $H: A \times[0,1] \rightarrow X$ такая, что $H(a, 0)=a$ для всех $a \in A$, и

$$
\int_{a}^{H(a, 1)} \omega \leqslant-N
$$

для всех $a \in A$.

Ниже мы будем предполагать, что форма $\omega$ фиксирована, а число $N$ большое или стремится к бесконечности. Это может случиться, только если класс когомологий $\xi=[\omega] \in H^{1}(X ; \mathbb{R})$ ненулевой. Действительно, очевидно, что в случае точной формы $\omega=d f$ непустое множество $A \subset X$ может быть $N$-подвижным относительно $\omega$ только при $N \leqslant \max f-\min f$.

Любое подмножество $A \subset X$, для которого вложение $A \rightarrow X$ стягиваемо, является $N$-подвижным относительно любой 1-формы $\omega$ в предположении, что когомологический класс $\xi=[\omega] \neq 0 \in H^{1}(X ; \mathbb{R})$ ненулевой.

Другой пример касается следующей ситуации. Пусть $X$ - замкнутое гладкое многообразие и $\omega$ - гладкая замкнутая 1-форма на $X$. Если у $\omega$ нет нулей, то любое подмножество $A \subset X$ является $N$-подвижным относительно $\omega$ для любого целого $N$. В этом случае гомотопия $H: A \times[0,1] \rightarrow X$ задается (нормированным надлежащим образом) градиентным потоком формы $\omega$ относительно римановой метрики на $X$.

В общем случае существуют топологические препятствия к подвижности подмножеств, которые улавливаются топологическими инвариантами типа категории Люстерника-Шнирельмана, которые мы опишем ниже.

6.2. Различные понятия категории. Приведем первую версию понятия категории $\operatorname{cat}(X, \xi)$ относительно когомологического класса. Определение, данное ниже, эквивалентно оригинальному определению из работы [15] (см. [51]).

ОПРЕДЕЛЕНИЕ 6.2. Пусть $X$ - конечный клеточный комплекс, $\xi \in H^{1}(X ; \mathbb{R})$. Зафиксируем замкнутую 1 -форму $\omega$, представляющую класс $\xi$. Тогда число

$$
\operatorname{cat}(X, \xi)
$$

определяется как минимальное целое $k$ такое, что для любого $N>0$ существует замкнутое подмножество $A, N$-подвижное относительно $\omega$, и $\operatorname{cat}_{X}(X-A) \leqslant k$.

Напомним, что для $A \subset X \operatorname{cat}_{X}(A)$ есть минимальное целое число $k$ такое, что $A$ можно покрыть $k$ открытыми подмножествами $X$, каждое из которых стягиваемо в $X$.

Заметим, что число $\operatorname{cat}(X, \xi)$ не зависит от выбора замкнутой 1-формы $\omega$ и может зависеть только от когомологического класса $\xi=[\omega] \in H^{1}(X ; \mathbb{R})$. Действительно, если $\omega^{\prime}$ - другая замкнутая 1-форма из того же класса когомологий $\xi=\left[\omega^{\prime}\right]$, то $\omega^{\prime}=\omega+d f$ для некоторой непрерывной функции $f: X \rightarrow \mathbb{R}$. 
Тогда для любого пути $\gamma:[a, b] \rightarrow X$ имеем

$$
\int_{\gamma} \omega^{\prime}=\int_{\gamma} \omega+f(\gamma(b))-f(\gamma(a)) .
$$

Поскольку $X$ компактно, то существует такая постоянная $C>0$, что для всех путей $\gamma$ из $X$ имеет место неравенство

$$
\left|\int_{\gamma} \omega^{\prime}-\int_{\gamma} \omega\right|<C .
$$

Следовательно, любое подмножество $A \subset X, N$-подвижное относительно формы $\omega$, является $(N-C)$-подвижным относительно $\omega^{\prime}$.

Может случиться, что

$$
\operatorname{cat}(X, \xi) \neq \operatorname{cat}(X,-\xi)
$$

(см. [13; пример 10.10]). Поэтому имеет смысл ввести (как впервые было предложено в [52]) симметрическую версию $\operatorname{cat}_{s}(X, \xi)$ понятия $\operatorname{cat}(X, \xi)$, более подходящую к динамическим приложениям.

ОПРедЕЛЕНИЕ 6.3. Пусть $X$ - конечный клеточный комплекс, $\xi \in H^{1}(X ; \mathbb{R})$. В классе когомологий $\xi$ зафиксируем представителя, замкнутую 1-форму $\omega$. Тогда число

$$
\operatorname{cat}_{s}(X, \xi)
$$

определяется как минимальное целое $k$ такое, что для любого $N>0$ существует замкнутое подмножество $A \subset X$, которое является $N$-подвижным как относительно $\omega$, так и относительно $-\omega$ и, кроме того, $\operatorname{cat}_{X}(X-A) \leqslant k$.

Очевидно, что

$$
\operatorname{cat}_{s}(X, \xi)=\operatorname{cat}_{s}(X,-\xi) \geqslant \max \{\operatorname{cat}(X, \xi), \operatorname{cat}(X,-\xi)\}
$$

Изменение кванторов в определении 6.2 дает несколько иное понятие категории $\operatorname{cat}^{1}(X, \xi)$, которое было изначально введено в [51]:

ОПРЕДЕЛЕНИЕ 6.4. Пусть $X$ - конечный клеточный комплекс, $\xi \in H^{1}(X ; \mathbb{R})$. Зафиксируем замкнутую 1-форму $\omega$, представляющую класс когомологий $\xi$. Тогда число $\operatorname{cat}^{1}(X, \xi)$ определяется как минимальное целое $k$ такое, что существует замкнутое подмножество $A \subset X$, которое является $N$-подвижным относительно $\omega$ для любого $N>0$, и при этом $\operatorname{cat}_{X}(X-A) \leqslant k$.

Для инварианта $\operatorname{cat}^{1}(X, \xi)$ также существует симметрическая модификация $\operatorname{cat}_{s}^{1}(X, \xi)$. Она определяется аналогично $\operatorname{cat}^{1}(X, \xi)$, с единственным отличием, что в определении 6.4 надо требовать существование замкнутого подмножества $A \subset X, N$-подвижного как относительно $\omega$, так и относительно $-\omega$.

Следующее определение дает другой, но близкий по духу инвариант.

ОПРЕДЕлЕНИЕ 6.5. Пусть $X$ - конечный клеточный комплекс, $\xi \in H^{1}(X ; \mathbb{R})$. Зафиксируем замкнутую 1 -форму $\omega$, представляющую класс $\xi$. Тогда $\operatorname{Cat}(X, \xi)$ определяется как минимальное целое число $k$, для которого существует открытое подмножество $U \subset X$, удовлетворяющее следующим условиям: 
(a) $\operatorname{cat}_{X}(X-U) \leqslant k$;

(b) для некоторой гомотопии $h: U \times[0, \infty) \rightarrow X$ выполняется

$$
h(x, 0)=x \quad \text { и } \quad \lim _{t \rightarrow \infty} \int_{x}^{h(x, t)} \omega=-\infty
$$

для любой точки $x \in U$;

(c) предел в (b) равномерный по $x \in U$.

Символ $\int_{x}^{h(x, t)} \omega$ обозначает интеграл $\int_{\gamma} \omega$, где путь $\gamma:[0, t] \rightarrow X$ задан формулой $\gamma(\tau)=h(x, \tau)$.

ЗАмечание 6.6. Приведенное выше определение $\operatorname{Cat}(X, \xi)$ отличается от изначального, предложенного в [16]; последнее было более симметричным и жестким для некоторых примеров.

Независимость $\operatorname{cat}^{1}(X, \xi)$ и $\operatorname{Cat}(X, \xi)$ от выбора замкнутой 1-формы $\omega$ из класса $[\omega]=\xi$ доказывается с помощью рассуждений, аналогичных приведенным при рассмотрении случая $\operatorname{cat}(X, \xi)$.

Какую роль играют инварианты $\operatorname{cat}(X, \xi), \operatorname{cat}^{1}(X, \xi)$ и $\operatorname{Cat}(X, \xi)$ в динамике, мы обсудим в последующих разделах.

Лемма 6.7. Для нулевого класса когомологий $\xi=0 \in H^{1}(X ; \mathbb{R})$ числа $\operatorname{cat}(X, \xi), \operatorname{cat}^{1}(X, \xi), \operatorname{Cat}(X, \xi)$ совпадают с классической категорией Люстерника-Шнирельмана $\operatorname{cat}(X)$ :

$$
\operatorname{cat}(X, \xi)=\operatorname{cat}^{1}(X, \xi)=\operatorname{Cat}(X, \xi)=\operatorname{cat}(X) .
$$

Доказательство. Докажем утверждение для $\operatorname{cat}(X, \xi)$. Если $\xi=0$, то можно выбрать $\omega=0$ и любое подмножество $A \subset X, N$-подвижное относительно $\omega$, является пустым при $N>0$. Следовательно, $\operatorname{cat}(X, \xi)=\operatorname{cat}_{X}(X)=$ $\operatorname{cat}(X)$.

ПримеР 6.8. Пусть $p: X \rightarrow S^{1}$ - локально тривиальное расслоение над окружностью и $\xi=p^{*}(\eta)$, где $\eta \in H^{1}\left(S^{1} ; \mathbb{R}\right), \eta \neq 0$. Тогда

$$
\operatorname{cat}(X, \xi)=\operatorname{cat}^{1}(X, \xi)=\operatorname{Cat}(X, \xi)=0
$$

(см. [13]). Заметим, что в этом примере cat $(X)$ может быть как угодно большой. Следовательно, новые "cat", не всегда равны $\operatorname{cat}(X)$.

Из сопоставления определений очевидно, что

$$
\operatorname{cat}(X, \xi) \leqslant \operatorname{cat}^{1}(X, \xi) \leqslant \operatorname{Cat}(X, \xi)
$$

Далее мы покажем, что $\operatorname{cat}(X, \xi)$ может быть существенно меньше, чем $\operatorname{cat}^{1}(X, \xi)$. У нас пока нет примеров, когда $\operatorname{cat}^{1}(X, \xi)<\operatorname{Cat}(X, \xi)$, но мы полагаем, что такие примеры существуют.

Лемма 6.9. Пусть $\xi \neq 0$ и $X-$ связное пространство. Тогда

$$
\operatorname{Cat}(X, \xi) \leqslant \operatorname{cat}(X)-1
$$


Доказательство можно найти в [13].

В качестве другого примера рассмотрим букет $X=Y \vee S^{1}$, где $Y$ - конечный полиэдр, и предположим, что класс $\xi \in H^{1}(X, \mathbb{R})$ удовлетворяет соотношениям $\left.\xi\right|_{Y}=0$ и $\left.\xi\right|_{S^{1}} \neq 0$. Можно показать (см. [13]), что тогда

$$
\operatorname{cat}(X, \xi)=\operatorname{cat}(Y)-1 \text {. }
$$

\section{3. Гомотопическая инвариантность.}

ПреДЛОЖЕНИЕ 6.10. Пусть $f: Y \rightarrow X$-гомотопическая эквивалентность между конечными связными клеточными комплексами и $\xi \in H^{1}(X ; \mathbb{R})$ - класс когомологий. Тогда

$$
\operatorname{cat}(X, \xi)=\operatorname{cat}\left(Y, f^{*} \xi\right) .
$$

Это утверждение останется верным, если заменить саt на cat $^{1}$ или Cat.

ДокАЗАТЕЛЬство. Пусть $\omega$ - замкнутая 1-форма на $X$ из когомологического класса $\xi$. Пусть $g: X \rightarrow Y$ и $K: X \times[0,1] \rightarrow X$ удовлетворяют соотношению $K: \operatorname{id}_{X} \simeq f g$. Так как $X$ компактно, то существует такая постоянная $C>0$, что

$$
\left|\int_{\alpha_{x}} \omega\right| \leqslant C
$$

для всех $x \in X$, где $\alpha_{x}(t)=K(x, t)$. Предположим, что подмножество $A \subset Y$ является $(N+C)$-подвижным относительно замкнутой 1-формы $\omega^{\prime}=f^{*} \omega$ на $Y$. Пусть $H: A \times[0,1] \rightarrow Y$ - такая гомотопия, что $H(a, 0)=a$ и $\int_{\gamma_{a}} \omega^{\prime} \geqslant N+C$ для всех $a \in A$, где $\gamma_{a}(t)=H(a, 1-t)$. Тогда множество $g^{-1}(A) \subset X$ является $N$-подвижным относительно $\omega$. Действительно, можно определить гомотопию $h_{t}: g^{-1}(A) \rightarrow X$ следующим образом:

$$
h_{t}(x)= \begin{cases}K(x, 2 t), & \text { если } 0 \leqslant t \leqslant 1 / 2, \\ f(H(g(x), 2 t-1)), & \text { если } 1 / 2 \leqslant t \leqslant 1,\end{cases}
$$

и для $x \in g^{-1}(A)$ имеем $\int_{\beta_{x}} \omega \geqslant N$, где $\beta_{x}(t)=h_{1-t}(x)$.

Если $\operatorname{cat}\left(Y, f^{*} \xi\right) \leqslant k$, то для любого $N$ существует замкнутое подмножество $A \subset Y,(N+C)$-подвижное относительно формы $\omega^{\prime}=f^{*} \omega$ и такое, что $\operatorname{cat}_{Y}(Y-A) \leqslant k$. Тогда подмножество $g^{-1}(A) \subset X$ является $N$-подвижным относительно $\omega$ и, как хорошо известно, $\operatorname{cat}_{X}\left(X-g^{-1}(A)\right) \leqslant k$. Это доказывает неравенство $\operatorname{cat}(X, \xi) \leqslant \operatorname{cat}\left(Y, f^{*} \xi\right)$.

Обратное неравенство доказывается аналогично, если $g f \simeq \mathrm{id}_{Y}$. Рассуждения для $\operatorname{cat}^{1}(X, \xi)$ и $\operatorname{Cat}(X, \xi)$ являются похожими. Предложение 6.10 доказано.

6.4. Пространства категории нуль. Приведем некоторые простые наблюдения о пространствах категории нуль. Более подробную информацию можно найти в $[52 ; \S 3]$.

Лемма 6.11. Пусть $X$ - конечный клеточный комплекса и $\xi \in H^{1}(X ; \mathbb{R})$ класс когомологий. Следующие свойства эквивалентны:

(a) $\operatorname{cat}(X, \xi)=0$;

(b) $\operatorname{cat}^{1}(X, \xi)=0$; 
(c) $\operatorname{Cat}(X, \xi)=0$;

(d) на пространстве $X$ существуют непрерывная замкнутая 1-форма $\omega$ из класса когомологий $\xi$ (в смысле $n$. 4.3) u гомотопия $h_{t}: X \rightarrow X$, $t \in[0,1]$, такие, что для любой точки $x \in X$ имеет место неравенство

$$
\int_{x}^{h_{1}(x)} \omega<0
$$

(в формуле (33) интеграл вичисляется вдоль кривой $t \mapsto h_{t}(x), t \in$ $[0,1])$

(е) для любой непрерьвной замкнутой 1-формы $\omega$ на пространстве $X$, представляющей класс $\xi$, существует такая гомотопия $h_{t}: X \rightarrow X$, $t \in[0,1]$, что для любой точки $x \in X$ выполняется неравенство (33).

ДокАЗАТЕЛьство. По определению 6.2 равенство $\operatorname{cat}(X, \xi)=0$ означает, что все пространство $X$ является $N$-подвижным для любого $N>0$, т. е. для любого $N>0$ существует такая гомотопия $H_{t}: X \rightarrow X, t \in[0,1]$, что для любого $x \in X$ выполнены условия $H_{0}(x)=x$ и

$$
\int_{x}^{H_{1}(x)} \omega<-N
$$

Следовательно, (а) влечет за собой (d).

В обратную сторону, имея свойство (d) и используя компактность $X$, находим такое $\varepsilon>0$, что (33) можно заменить на условие $\int_{x}^{h_{1}(x)} \omega<-\varepsilon$. Теперь можно итерировать эту деформацию: $k$-я итерация является гомотопией $H_{t}^{k}: X \rightarrow X, t \in[0,1]$, определенной следующим образом. Пусть $h_{1}^{(i)}: X \rightarrow X$ обозначает $i$-кратную композицию $h_{1}^{(i)}=h_{1} \circ h_{1} \circ \cdots \circ h_{1}(i$ раз $)$. Тогда для $t \in[i / k,(i+1) / k]$ имеем

$$
H_{t}^{k}(x)=h_{k t-i}\left(h_{1}^{(i)}(x)\right)
$$

Если для любого $x \in X$ верно $\int_{x}^{h_{1}(x)} \omega<-\varepsilon$, то для $k$-й итерации получаем $\int_{x}^{H_{1}^{k}(x)} \omega<-k \varepsilon$ и $(\mathrm{d})$ следует из предположения $k>N / \varepsilon$. Тем самым показана эквивалентность (а) и $(d)$.

Импликация (e) $\Rightarrow(\mathrm{d})$ очевидна. Предположим, что для формы $\omega$ имеет место $(\mathrm{d})$, и пусть $\omega_{1}$ - другая непрерывная замкнутая 1-форма из того же класса когомологий, т. е. $\omega_{1}=\omega+d f$ для некоторой непрерывной функции $f: X \rightarrow \mathbb{R}$. Используя компактность пространства $X$, можно найти такое число $C$, что для любого пути $\gamma:[0,1] \rightarrow X$ имеем $\left|\int_{\gamma} d f\right|<C$. Зафиксируем $N>C$ и применим эквивалентность между условиями (а) и (с) для нахождения гомотопии $h_{t}: X \rightarrow X$ такой, что $\int_{x}^{h_{1}(x)} \omega<-N$. Тогда $\int_{x}^{h_{1}(x)} \omega_{1}<0$, т. е. выполняется
условие $($ е $)$.

Очевидно, что из условия (b) следует (a). Таким образом, осталось показать, что из (a) следует (b). Пусть верно (b), зафиксируем описанную в (c) 
деформацию. Пусть $C>0$ - такое число, что для любых $x \in X$ и $t \in[0,1]$ имеет место $\int_{1}^{h_{t}(x)} \omega<C$. Тогда для любой итерации $H_{t}^{k}: X \rightarrow X$ (см. выше) имеем $\int_{x}^{H_{t}^{k}(x)} \omega<C$, откуда и следует утверждение. Лемма доказана.

В случае замкнутого гладкого многообразия $X$ деформацию, возникшую в пункте (b), можно построить как поток, порожденный векторным полем $v$ на многообразии $X$, удовлетворяющим условию $\omega(v)<0$.

Замечание из предыдущего абзаца объясняет, почему следующее утверждение можно трактовать как аналог классической теоремы Эйлера-Пуанкаре.

TeOpema 6.12. Ecлu $\operatorname{cat}(X, \xi)=0$, mo $\chi(X)=0$.

ДоказАТельство. Пусть $\operatorname{cat}(X, \xi)=0$. Тогда $\xi \neq 0$, т. е. ранг $r$ класса когомологий $\xi$ положителен: $r>0$. Согласно приводимой ниже лемме 12.2 , существует $\xi$-трансцендентное расслоение $L \in \mathscr{V}_{\xi}=\left(\mathbb{C}^{*}\right)^{r}$. Если $H^{q}(X ; L) \neq 0$ для некоторого $q$, то применение приводимой ниже теоремы 12.4 с $k=0$ даст неравенство $\operatorname{cat}(X, \xi)>0$, что противоречит нашему предположению. Следовательно, $H^{q}(X ; L)=0$ для всех $q$, откуда следует, что

$$
\chi(X)=\sum_{q}(-1)^{q} \operatorname{dim} H^{q}(X ; L)=0 .
$$

Теорема доказана.

Пары $(X, \xi)$ с нулевой категорией $\operatorname{cat}(X, \xi)=0$ образуют “идеал" в следующем смысле.

Лемма 6.13. Пусть $X_{1}$ и $X_{2}$ - конечные клеточные комплексъ и $\xi_{1} \in$ $H^{1}\left(X_{1} ; \mathbb{R}\right), \xi_{2} \in H^{1}\left(X_{2} ; \mathbb{R}\right)$ - классы одномерных когомологий. Если выполнено равенство $\operatorname{cat}\left(X_{1}, \xi_{1}\right)=0$, то $\operatorname{cat}\left(X_{1} \times X_{2}, \xi\right)=0$, где $\xi=\xi_{1} \times 1+1 \times \xi_{2} \in$ $H^{1}\left(X_{1} \times X_{2} ; \mathbb{R}\right)$.

ДокАЗАтЕльство. Утверждение следует непосредственно из определений.

\section{7. Эффект фокусирования}

В этом разделе мы приступаем к рассмотрению приложения инварианта $\operatorname{cat}(X, \xi)$ в динамике. Опишем эффект фокусирования, открытый в [15], см. также [13].

Природа теории Люстерника-Шнирельмана для замкнутых 1-форм существенно отличается как от классической теории Люстерника-Шнирельмана для функций, так и от теории Новикова. Как хорошо известно, в произвольном ненулевом классе когомологий $\xi \in H^{1}(M ; \mathbb{Z}), \xi \neq 0$, всегда можно найти замкнутую 1-форму с не более чем одним (возможно, сильно вырожденным) нулем. Следовательно, количественную оценку числа нулей можно получить только при некоторых дополнительных предположениях. Оказывается, что эти дополнительные предположения могут быть выражены в терминах некоторых интересных динамических свойств градиентоподобных векторных полей, задаваемых замкнутой 1-формой. Это делает новую теорию потенциально очень полезной для динамики. 

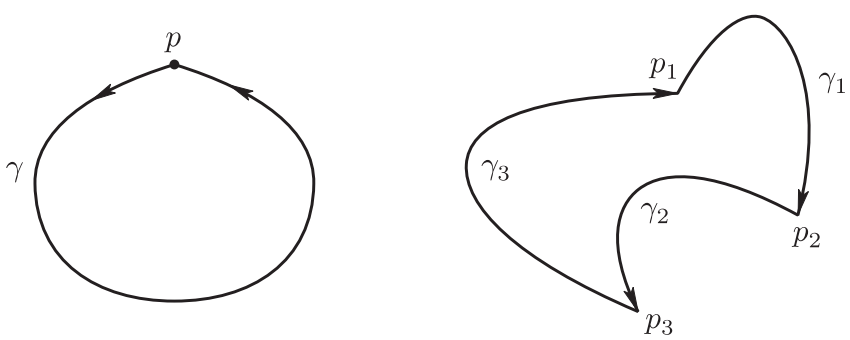

Рис. 7. Гомоклиническая орбита (слева) и гомоклинический цикл (справа)

Пусть $v$ - гладкое векторное поле на замкнутом гладком многообразии $M$. Напомним, что гомоклиническая орбита поля $v$ определяется как интегральная траектория $\gamma(t)$,

$$
\dot{\gamma}(t)=v(\gamma(t)), \quad t \in \mathbb{R},
$$

для которой пределы $\lim _{t \rightarrow+\infty} \gamma(t)$ и $\lim _{t \rightarrow-\infty} \gamma(t)$ существуют и равны:

$$
\lim _{t \rightarrow+\infty} \gamma(t)=\lim _{t \rightarrow-\infty} \gamma(t)
$$

Более общо, гомоклиническим ииклом длины $n$ называется такая последовательность интегральных траекторий $\gamma_{1}(t), \gamma_{2}(t), \ldots, \gamma_{n}(t)$ поля $v$, что выполнены следующие условия:

$$
\lim _{t \rightarrow+\infty} \gamma_{i}(t)=p_{i+1}=\lim _{t \rightarrow-\infty} \gamma_{i+1}(t)
$$

при $i=1, \ldots, n-1$ и

$$
\lim _{t \rightarrow+\infty} \gamma_{n}(t)=p_{1}=\lim _{t \rightarrow-\infty} \gamma_{1}(t)
$$

Гомоклинические орбиты были открыты Пуанкаре и изучались Смейлом. В математической литературе очень много результатов о существовании гомоклинических орбит для гамильтоновых систем. По очевидной причине гомоклинические орбиты не могут возникать для градиентных систем функций, рассматриваемых в классической теории Люстерника-Шнирельмана.

Одна из основных теорем этого раздела (теорема 7.2 , изначально доказанная в [15]) утверждает, что любая гладкая замкнутая 1-форма $\omega$ на гладком замкнутом многообразии $X$ имеет не меньше $\operatorname{cat}(X, \xi)$ геометрически различных нулей $\left(\right.$ где $\xi=[\omega] \in H^{1}(X ; \mathbb{R})$ обозначает когомологический класс $\omega$ ) в предположении, что $\omega$ допускает градиентоподобное векторное поле без гомоклинических циклов.

С другой стороны, основной результат этого раздела утверждает, что у любого градиентоподобного векторного поля 1-формы $\omega$ есть гомоклинический цикл, если количество нулей $\omega$ меньше чем $\operatorname{cat}(M, \xi)$.

ОПРЕДЕЛЕНИЕ 7.1. Пусть $M$ - гладкое замкнутое многообразие и $\omega$ - гладкая замкнутая 1-форма на $M$ с множеством нулей

$$
Y_{\omega}=\left\{p \in M ; \omega_{p}=0\right\} .
$$


Будем говорить, что гладкое векторное поле $v$ на $M$ является градиентоподобнысм для 1-формы $\omega$, если $\omega$ является 1-формой Ляпунова для пары $\left(\Phi, Y_{\omega}\right)$, где $\Phi$ - поток на многообразии $M$, порожденный полем $-v$ (см. определение 5.1).

Заметим, что отсюда следует, что

$$
\omega(v)>0
$$

на $M-Y_{\omega}$ и множество $Y_{\omega}$ инвариантно относительно потока, порожденного полем $v$.

В частном случае, когда множество нулей $Y_{\omega}$ конечно, векторное поле $v$ является градиентоподобным для $\omega$ тогда и только тогда, когда множества нулей $\omega$ и $v$ совпадают и на множестве $M-Y_{\omega}$ выполняется неравенство (36).

Теорема 7.2 [15]. Пусть $\omega$ - гладкая замкнутая 1-форма на замкнутом гладком многообразии $M$. Если $\omega$ допускает градиентоподобное векторное поле $v$ без гомоклинических ииклов, то $\omega$ имеет не менее $\operatorname{cat}(M,[\omega])$ геометрически различных нулей.

Здесь $[\omega] \in H^{1}(M ; \mathbb{R})$ обозначает когомологический класс формы $\omega$.

Переформулируем вышеприведенную теорему.

Теорема 7.3. Если количество нулей гладкой замкнутой 1-формы $\omega$ менъше чем $\operatorname{cat}(M,[\omega])$, то любое градиентоподобное векторное поле $v$ формы $\omega$ имеет гомоклинический иикл.

Заметим, что определение градиентоподобного векторного поля в этой работе несколько более общее, чем в [15], [13].

Чуть более информативное утверждение доказано в [13]:

Теорема 7.4 [13; теорема 10.16]. Пусть гладкая замкнутая 1-форма $\omega$ на замкнутом многообразии $M$ имеет менъше чем $\operatorname{cat}(M, \xi)$ нулей, где $\xi=$ $[\omega] \in H^{1}(M ; \mathbb{R})$. Тогда существует такое целое число $N>0$, что у любого градиентоподобного векторного поля $v$ формы $\omega$ естъ гомоклинический иикл $\gamma_{1}, \ldots, \gamma_{n}$, удовлетворяющий неравенству

$$
\sum_{i=1}^{n} \int_{\gamma_{i}} \omega \leqslant N .
$$

Сопоставим эти результаты с теоремой 3.1, которая гарантирует существование замкнутой 1-формы с не более чем одним нулем в любом ненулевом целочисленном когомологическом классе. Это показывает, что всегда существуют гомоклинические циклы, которые устойчивы относительно возмущения градиентоподобного векторного поля!

Этот “эффект фокусирования" начинается, когда число нулей замкнутой 1-формы становится меньше $\operatorname{cat}(M, \xi)$. Это новое явление, которое не проявлялось в теории Новикова. Действительно, если предположить, что все нули формы $\omega$ морсовского типа, то (по теореме Купки-Смейла [53]) для формы $\omega$ всегда можно найти такое градиентоподобное векторное поле $v$, что интегральные траектории, соединяющие два нуля, идут из нуля более высокого индекса к нулю с меньшим индексом; у такого векторного поля $v$ нет гомоклинических циклов. 
Приведем как иллюстрацию утверждение, которое является следствием теоремы 7.3 и зависит от вычисления $\operatorname{cat}(X, \xi)$ в случае, когда $X$ - произведение поверхностей (см. теорему 14.12 ниже).

ТеОрема 7.5. Пусть $M^{2 k}$ - произведение $\Sigma_{1} \times \Sigma_{2} \times \cdots \times \Sigma_{k}$ замкнутых ориентированных поверхностей $\Sigma_{i}$ рода $g_{i}>1$. Для когомологического класса $\xi=[\omega] \in H^{1}(X ; \mathbb{R})$ обозначим $r(\xi)$ количество индексов $i \in\{1, \ldots, k\}$, для которых $\left.\xi\right|_{\Sigma_{i}}=0$. Пусть $\omega$ - замкнутая гладкая 1-форма на $M$ из класса когомологий $\xi$, имеющая не более $2 r(\xi)$ нулей. Тогда любое градиентоподобное поле формы $\omega$ имеет гомоклинический цикл.

Теорема 7.3 была обобщена в [54], где изучался более общий случай замкнутых 1-форм с бесконечным количеством нулей. Результат, полученный в [54], "сравнивает" топологию множества нулей формы $\omega$ и инвариант $\operatorname{cat}(M,[\omega])$. В разделе 9 настоящей статьи мы докажем следующий усиленный вариант основной теоремы работы [54] и теоремы 7.4.

Теорема 7.6. Пусть $\omega$ - гладкая замкнутая 1-форма на гладком замкнутом многообразии $M$. Предположим, что множество нулей $Y=\{p \in M$; $\left.\omega_{p}=0\right\}$ имеет такую окрестность $U, Y \subset U \subset M$, что $\left.\omega\right|_{U}$ есть точная форма ${ }^{3}$ и $Y$ состоит из конечного числа компонент связности $Y_{1}, \ldots, Y_{k}$, для которых

$$
\sum_{i=1}^{k} \operatorname{cat}_{M}\left(Y_{i}\right)<\operatorname{cat}(M,[\omega])
$$

где $[\omega] \in H^{1}(M ; \mathbb{R})$ обозначает класс когомологий $\omega$. Тогда найдется такое целое $N>0$, что для любого градиентоподобного векторного поля $v$ бормы $\omega$ существуют конечная чепочка орбит

$$
\gamma_{1}, \ldots, \gamma_{\ell}, \gamma_{\ell+1}=\gamma_{1}
$$

лежащих в $M-Y($ при $1 \leqslant \ell \leqslant k)$, и метки $1 \leqslant i(j) \leqslant k$ такие, что

$$
\bigcap_{t \in \mathbb{R}} \overline{\gamma_{j}([t,+\infty))} \subset Y_{i(j)} \quad u \bigcap_{t \in \mathbb{R}} \overline{\gamma_{j+1}((-\infty, t])} \subset Y_{i(j)}
$$

для всех $1 \leqslant j \leqslant \ell, u$

$$
\sum_{i=1}^{\ell} \int_{\gamma_{i}} \omega \leqslant N
$$

Другими словами, если множество нулей замкнутой 1-формы $\omega$ является "малым" в некотором смысле, т. е. удовлетворяет неравенствам (38), то любое градиентоподобное векторное поле $v$ формы $\omega$ имеет обобщенный гомоклинический цикл, который делает не более $N$ полных поворотов относительно $\omega$.

Заметим, что условия (39) описывают обобщение понятия гомоклинического цикла. Действительно, условия (39) интуитивно означают, что $j$-я траектория $\gamma_{j}$ "кончается", в той же компоненте связности $Y_{i(j)}$ множества $Y$, в которой

\footnotetext{
3 Это условие автоматически выполнено, если когомологический класс $[\omega]$ является целочисленным или $Y$ есть $\operatorname{ENR}$ (см. леммы 5.2, 5.3).
} 


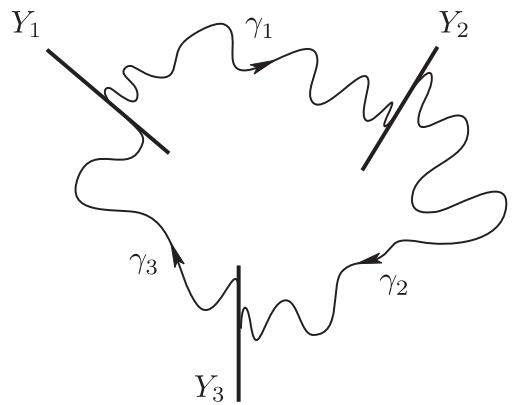

Рис. 8. Обобщенный гомоклинический цикл

“начинается" $(j+1)$-я траектория $\gamma_{j+1}$. Такую последовательность траекторий $\gamma_{1}, \ldots, \gamma_{\ell}$ будем называть обобщенным гомоклиническим ииклом (см. рис. 8).

Теорема 7.6 сводится к теореме 7.2 при дополнительном требовании конечности множества $Y$.

Доказательство теоремы 7.6 отложим до раздела 9; в следующем ниже разделе докажем теорему, которая будет использована в разделе 9.

\section{8. Существование окрестностей, выпуклых относительно потока}

В этом разделе мы изучим некоторую вспомогательную задачу, которая будет использована в доказательстве теорем 7.6 и 10.1, а также представляет самостоятельный интерес. Результаты этого раздела типичны для теории Конли об изолированных инвариантных множествах. Мы будем следовать работе [51].

Рассмотрим гладкое векторное поле $v$ на замкнутом гладком многообразии $M$. Пусть $\Phi: M \times \mathbb{R} \rightarrow M-$ поток, порожденный $v$. Будем записывать $\Phi(x, t)$ как $x \cdot t$, где $x \in M$ и $t \in \mathbb{R}$. Символ $R$ обозначает цепно-рекуррентное множество потока $\Phi$.

Теорема 8.1 [51]. Пусть $Z$ - компонента связности множества $R$, которая изолирована в $R$, m.е. существует такая окрестность $Z \subset U$, что $U \cap R=Z$. Пусть $W \subset M$ - окрестность $Z$. Тогда существует открьтая окрестность $B$ множества $Z$, содержащаяся в $W$, со следующими двумя свойствами.

(А) Для любого $x \in M$ открытое множество

$$
J_{x}=\{t ; x \cdot t \in B\} \subset \mathbb{R}
$$

выпукло (т.е. является пустым множеством или интервалом);

(В) Пусть $A$ - множество точек $x \in M$, для которьх интервал $J_{x}$ непуст и ограничен снизу, тогда функция

$$
A \rightarrow \mathbb{R}, \quad x \mapsto \inf J_{x}
$$

непрерывна.

Окрестность $B$ множества $Z$, обладающая свойствами (А) и (В), называется выпуклой относительно потока $\Phi$. 


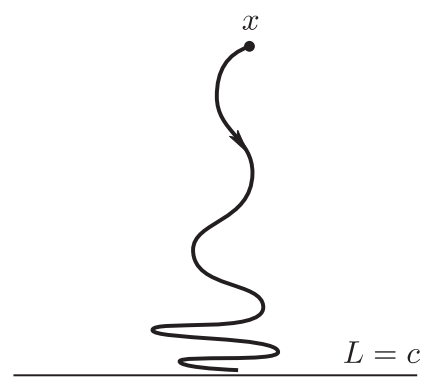

Рис. 9

Теорему 8.1 можно сопоставить с леммой В.1 из приложения В работы [15]. Согласно теореме Конли [21], [22], для потока существует гладкая функция Ляпунова $L: M \rightarrow \mathbb{R}$ (см. также [18; предложение 2], где дано доказательство гладкой версии теоремы Конли). Функция Ляпунова $L$ удовлетворяет следующим условиям: $V(L)<0$ на дополнении $M-R$ к $R$ и ее дифференциал $d L$ обращается в нуль на $R$.

Зафиксируем точку $x \in M-R$ и рассмотрим ее орбиту $x(t)=x \cdot t$. Функция $t \mapsto L(x \cdot t)$ является строго убывающей. Следовательно, при $t$, стремящемся $\mathrm{K}+\infty$, предел

$$
\ell(x)=\lim _{t \rightarrow \infty} L(x \cdot t)
$$

существует и является конечным (см. рис. 9). При $x \in R$ функция $t \mapsto L(x \cdot t)$ постоянна.

Для любого $x \in M$ число $\ell(x)$ является критическим значением $L$. В самом деле, $\omega$-предельное множество ${ }^{4} \omega(x)$ содержится в множестве уровня $L^{-1}(\ell(x))$, а с другой стороны, $\omega(x)$ является частью цепно-рекуррентного множества $R$, которое и есть множество критических точек для $L$.

ЛЕмма 8.2. Функиия $\ell: M \rightarrow \mathbb{R}$ является полунепрерьвной сверху: если $x_{n} \rightarrow x \in M, m o$

$$
\ell(x) \geqslant \lim \sup \ell\left(x_{n}\right)
$$

ДокАЗАТЕльство. Для любого $\varepsilon>0$ существует $T>0$ такое, что $0<$ $L(x \cdot T)-\ell(x)<\varepsilon$. Поскольку $L$ непрерывна и отображение $x \mapsto x \cdot T$ непрерывно, существует такая окрестность $U \subset M$ точки $x$, что $|L(y \cdot T)-L(x \cdot T)|<\varepsilon$ для любого $y \in U$. Следовательно, для всех $y \in U$ имеем

$$
\ell(y) \leqslant L(y \cdot T)<L(x \cdot T)+\varepsilon<\ell(x)+2 \varepsilon .
$$

Отсюда получаем неравенство $\ell(y) \leqslant \ell(x)$. Лемма доказана.

Ограничение функции $L: M \rightarrow \mathbb{R}$ на множество $Z$ постоянно. Действительно, $L(Z) \subset \mathbb{R}$ связно (как образ связного множества) и согласно теореме Сарда имеет меру нуль. Поэтому оно состоит из одной точки. Обозначим $c=L(Z)$.

Пусть $Z \subset W$ - открытая окрестность. Поскольку $Z$ изолировано в $R$ (по предположению), без ограничения общности можно считать, что $\bar{W} \cap R=Z$.

\footnotetext{
${ }^{4}$ Напомним, что $\omega$-предельное множество $\omega(x)$ определяется как $\bigcap_{T \in \mathbb{R}} \overline{\{x \cdot t ; t \geqslant T\}}$.
} 
Зафиксируем $\varepsilon>0$ и обозначим $A_{+}(\varepsilon)$ множество всех точек $x \in M$ со следующими свойствами: (a) $L(x)=c+\varepsilon$; (b) $\omega(x) \subset Z$. Заметим, что из (b) следует $\ell(x)=c$.

Лемма 8.3. Для любого достаточно малого $\varepsilon>0$ множество $A_{+}(\varepsilon)$ содержится в окрестности $W$.

ДоказАтельство. Предположим противное: пусть существует такая сходящаяся последовательность $x_{n} \in M-W$, что $L\left(x_{n}\right) \rightarrow c, L\left(x_{n}\right)>c, \ell\left(x_{n}\right)=c$ и $\omega\left(x_{n}\right) \subset Z$, где $\omega(x)$ обозначает $\omega$-предельное множество траектории $x \cdot t$. Если $x_{0}=\lim x_{n} \in M$ является пределом $x_{n}$, то (используя (42)) получаем $\ell\left(x_{0}\right) \geqslant c$. С другой стороны, $L\left(x_{0}\right)=c$ и, следовательно, $\ell\left(x_{0}\right)=c$. Получаем, что точка $x_{0}$ принадлежит множеству $L^{-1}(c) \cap(R-Z)$. Другими словами, она лежит в компоненте связности $R$, отличной от $Z$.

Зададим риманову метрику на $M$. Обозначим $d>0$ расстояние между $Z$ и $R-Z$. Пусть $K>0$ - такая константа, что норма вектора $V$ меньше $K$ в каждой точке многообразия $M$. Такое $K$ существует ввиду компактности $M$.

Зафиксируем $\delta>0$ так, что $\delta<d / 2$. Ограничение функции $V(L): M \rightarrow \mathbb{R}$ на дополнение $\delta$-окрестности множества $R$ отрицательно и, более того, ограничено сверху: $V(L) \leqslant-\eta$ для некоторого положительного $\eta=\eta_{\delta}>0$. Теперь мы можем найти такое большое $n$, что

$$
L\left(x_{n}\right)-c<\frac{\eta(d-2 \delta)}{K}
$$

и $x_{n}$ лежит в $\delta$-окрестности множества $R-Z$. Траектория $x_{n} \cdot t$ приближается к $Z$ при больших $t$. Длина траектории задается формулой

$$
\int_{t_{1}}^{t_{2}}|\dot{x}(t)| d t=\int_{t_{1}}^{t_{2}}|V(x(t))| d t \leqslant K\left|t_{2}-t_{1}\right| .
$$

Отсюда можно сделать вывод, что время $\tau_{n}$, которое траектория $x_{n} \cdot t$ при $t>0$ проводит в дополнении к $\delta$-окрестности $R$, оценивается следующим образом:

$$
\tau_{n} \geqslant \frac{d-2 \delta}{K}
$$

Поэтому при больших $t>0$ имеем

$$
L\left(x_{n} \cdot t\right)-L\left(x_{n}\right)=\int_{0}^{t} V(L)\left(x_{n} \cdot t\right) d t \leqslant-\eta \cdot \tau_{n} \leqslant-\frac{\eta(d-2 \delta)}{K} .
$$

Эти неравенства показывают (поскольку $L\left(x_{n}\right)$ стремится к $c$ ), что при больших $t$ имеем $L\left(x_{n} \cdot t\right)<c$, что противоречит предположению $\ell\left(x_{n}\right)=c$. Лемма доказана.

ЗАмечание 8.4. Определим $A_{-}(\varepsilon)$ как множество всех точек $x \in M$ таких, что $L(x)=c-\varepsilon$ и $\alpha(x) \subset Z$, где $\alpha(x)$ обозначает $\alpha$-предельное множество траектории $x \cdot t$. Применяя приведенное выше рассуждение к потоку с обращенным временем, получаем, что для любой окрестности $W$ множества $Z$ множество $A_{-}(\varepsilon)$ содержится в $W$ при достаточно малых $\varepsilon>0$. 


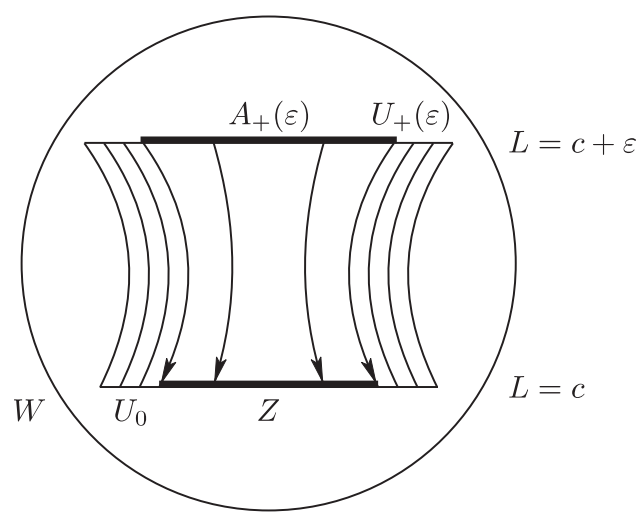

Рис. 10

Схематично это доказательство можно представить следующим образом: точка $x_{n}$ расположена очень близко от компоненты связности $Z^{\prime}$ множества $R$, лежащей в $L^{-1}(c)$ и отличной от $Z$. Траектория с началом в $x_{n}$ не может приближаться к множеству $Z$, поскольку по мере того как она преодолевает расстояние между $Z$ и $Z^{\prime}$, она опускается относительно $L$ так, что точка $x_{n} \cdot t$ соскальзывает ниже уровня $c$.

Лемма 8.5. Для любого достаточно малого $\varepsilon>0$ множество $A_{+}(\varepsilon)$ является замкнутым.

ДокАЗАТЕЛЬство. Пусть $W$ - окрестность множества $Z$ и $\bar{W} \cap R=Z$. Пусть также $\varepsilon>0$ таково, что $A_{+}(\varepsilon) \subset W$. Предположим, что последовательность $x_{n} \in A_{+}(\varepsilon)$ сходится к точке $x_{0}$. Тогда для любого $t>0$ точка $x_{n} \cdot t$ лежит в $W$. Следовательно, для любых $t>0$ точка $x_{0} \cdot t$ принадлежит $\bar{W}$. Отсюда вытекает, что $\omega\left(x_{0}\right) \subset \bar{W} \cap R=Z$. Поэтому $x_{0}$ является элементом $A_{+}(\varepsilon)$. Лемма доказана.

Лемма 8.6. Пусть $W$ - такая открытая окрестность $Z$, что $\bar{W} \cap R=Z$. Пусть $\varepsilon>0$ таково, что $A_{+}\left(\varepsilon^{\prime}\right)$ содержится в $W$ при любых $0<\varepsilon^{\prime} \leqslant \varepsilon$. Тогда существуют открытая окрестность $U_{+}(\varepsilon)$ множества $A_{+}(\varepsilon)$ в множестве уровня $L^{-1}(c+\varepsilon)$ и открытая окрестность $U_{0}$ множества $Z$ в $L^{-1}(c)$ со следующими свойствами:

1) если $x \in U_{+}(\varepsilon)-A_{+}(\varepsilon)$, то для некоторого $\tau_{x}>0$ точка $x \cdot \tau_{x}$ лежит в $U_{0}-Z$;

2) отображение $x \mapsto x \cdot \tau_{x}$ является гомеоморбизмом $U_{+}(\varepsilon)-A_{+}(\varepsilon) \rightarrow$ $U_{0}-Z$

3) для любого $0 \leqslant t \leqslant \tau_{x}$ точка $x \cdot t$ лежит в $W$;

4) число $\tau_{x}$ непрерывно зависит от $x \in U_{+}(\varepsilon)-A_{+}(\varepsilon)$ и стремится $\kappa+\infty$, когда $x \in U_{+}(\varepsilon)-A_{+}(\varepsilon)$ приближсается $\kappa A_{+}(\varepsilon)$.

ДокАЗАТЕЛЬСтво. Если $L(y)=c+\varepsilon$ и точка $y$ находится достаточно близко от множества $A_{+}(\varepsilon)$, то при $t>0$ орбита $y \cdot t$ остается на $W$, пока не покинет множество $L \geqslant c$. Действительно, если это утверждение неверно, то существуют последовательности точек $y_{n} \in M$ и чисел $t_{n}>0$ такие, что $L\left(y_{n}\right)=c+\varepsilon$, 
$y_{n} \rightarrow x \in A_{+}(\varepsilon), L\left(y_{n} \cdot t_{n}\right) \geqslant c$ и $y_{n} \cdot t_{n} \notin W$. Зафиксируем риманову метрику на $M$. Пусть $K>0$ таково, что длина вектора $V(x)$ не превосходит $K$ в каждой точке $x \in M$. Выберем малую окрестность $G$ множества $Z$, замыкание которой содержится в $W$. Пусть $d>0$ - расстояние между $\bar{G}$ и $M-W$. На множестве $W-G$ функция $V(L)$ удовлетворяет неравенству $V(L) \leqslant-\eta$ для некоторого $\eta>0$. Пусть теперь $G^{\prime} \subset G$ - такая окрестность $Z$, что выполнено

$$
L\left(G^{\prime}\right) \subset\left(-\infty, c+\eta \cdot \frac{d}{K}\right) .
$$

Поскольку $x$ принадлежит $A_{+}(\varepsilon)$, существует такое $t^{\prime}>0$, что $x \cdot t^{\prime} \in G^{\prime}$. Тогда для достаточно больших $n$ верно $y_{n} \cdot t^{\prime} \in G^{\prime}$. Рассуждая как в доказательстве леммы 8.3 , получаем, что точка $y_{n} \cdot t$ не может достичь множества $M-W$, пока не покинет область $L \geqslant c$.

Аналогично: если $L(y)=c$, где $y \in W-Z$ достаточно близко к $Z$, то траектория $y \cdot t$ для некоторого $t<0$ достигает уровня $L=c+\varepsilon$, не покидая $W$. Действительно, если это утверждение неверно, то найдется такая последовательность $y_{n} \in W-Z, L\left(y_{n}\right)=c$, что $y_{n} \rightarrow x \in Z$ и для некоторого $t_{n}<0$ имеем $y_{n} \cdot t_{n} \in \partial W$ и $L\left(y_{n} \cdot t_{n}\right) \leqslant c+\varepsilon$. Мы можем предположить, что последовательность $y_{n} \cdot t_{n}$ сходится к точке $z \in \partial W$. Если $\ell(z)>c$, то траектория $z \cdot t$, где $t>0$, пересекает поверхность уровня $L=c$ в точке, которая не принадлежит $Z$, и траектория $y_{n} \cdot t$ должна при $n \rightarrow \infty$ пересечь этот уровень в некоторой близлежащей точке. Получили противоречие. Следовательно, осталось рассмотреть случай $\ell(z)=c$, который допускает две возможности: либо $\omega(z)$ содержится в отличной от $Z$ компоненте связности $R$, которая также принадлежит поверхности уровня $L=c$, либо $\omega(z) \subset Z$. Первая возможность приведет к противоречию, если повторить рассуждение из доказательства леммы 8.3. Вторая возможность означает, что $z$ принадлежит $A_{+}\left(\varepsilon^{\prime}\right)$ при $0<\varepsilon^{\prime} \leqslant \varepsilon$. Это является противоречием, поскольку мы предположили, что $A_{+}\left(\varepsilon^{\prime}\right)$ содержится в открытом множестве $W$.

Пусть $U_{0}$ - объединение $Z$ и множества всех точек $y \in W \cap L^{-1}(c)$ таких, что траектория $y \cdot t$ при $t<0$ достигает $W \cap L^{-1}(c+\varepsilon)$, не покидая $W$.

Пусть $U_{+}(\varepsilon)$ - объединение $A_{+}(\varepsilon)$ и множества всех $y \in W \cap L^{-1}(c+\varepsilon)$, для которых траектория $y \cdot t$ при $t>0$ достигает поверхность уровня $L^{-1}(c)$, оставаясь в $W$.

Как показано выше, множество $U_{0}$ является открытой окрестностью множества $Z$ в $L^{-1}(c)$ и $U_{+}(\varepsilon)$ - открытая окрестность $A_{+}(\varepsilon)$ в $L^{-1}(c+\varepsilon)$. Нетрудно видеть, что утверждение леммы 8.6 верно для $U_{0}$ и $U_{+}(\varepsilon)$. Покажем, например, что если $x_{n} \in U_{+}(\varepsilon)-A_{+}(\varepsilon)$ и $x_{n} \rightarrow x \in A_{+}(\varepsilon)$, то $\tau_{x_{n}}$ стремится к $+\infty$. Если это не так, то можно выбрать подпоследовательность последовательности $\tau_{x_{n}}$, имеющую конечный предел $\tau$. Тогда $L(x \cdot \tau)=\lim L\left(x_{n} \cdot \tau_{x_{n}}\right)=c$. Следовательно, траектория $x \cdot t$ достигает $Z$ за конечное время, что невозможно, поскольку $Z$ инвариантно относительно потока и $x \notin Z$. Лемма доказана.

Приведем аналог леммы 8.6.

Лемма 8.7. Пусть $W$ - такая открытая окрестность множества $Z$, для которой $\bar{W} \cap R=Z$. Виберем $\varepsilon>0$ так, чтобы $A_{-}\left(\varepsilon^{\prime}\right)$ содержалось в $W$ 
для всех $0<\varepsilon^{\prime} \leqslant \varepsilon$. Тогда существуют открытая окрестность $U_{-}(\varepsilon)$ множества $A_{-}(\varepsilon)$ в множестве уровня $L^{-1}(c-\varepsilon)$ и открытая окрестность $U_{0}$ множества $Z$ в $L^{-1}(c)$ со следующими свойствами:

1) если $x \in U_{-}(\varepsilon)-A_{-}(\varepsilon)$, то для некоторого $T_{x}<0$ точка $x \cdot T_{x}$ лежит в $U_{0}-Z$;

2) отображение $x \mapsto x \cdot T_{x}$ является гомеоморфизмом

$$
U_{-}(\varepsilon)-A_{-}(\varepsilon) \rightarrow U_{0}-Z
$$

3) для любого $T_{x}<t<0$ точка $x \cdot t$ принадлежит $W$;

4) число $T_{x}$ непрерьвно зависит от $x \in U_{-}(\varepsilon)-A_{-}(\varepsilon)$ и стремится $\kappa-\infty$, когда $x \in U_{-}(\varepsilon)-A_{-}(\varepsilon)$ приближается $\kappa A_{-}(\varepsilon)$.

ДокАЗАТЕЛЬСТво аналогично доказательству леммы 8.6.

ДоКАЗАТЕЛЬСТВо теОРЕМЫ 8.1. Пусть $W \subset M$ - открытая окрестность $Z$. Без потери общности можно предположить, что $\bar{W} \cap R=Z$. Согласно лемме 8.3 и замечанию 8.4 , найдется такое $\varepsilon>0$, что для любого $0<\varepsilon^{\prime} \leqslant \varepsilon$ множества $A_{+}\left(\varepsilon^{\prime}\right)$ и $A_{-}\left(\varepsilon^{\prime}\right)$ содержатся в $W$.

Пусть $C$ обозначает множество всех точек $x \in W \cap L^{-1}(c-\varepsilon, c+\varepsilon)$, для которых существуют числа $t_{x}<0<\tau_{x}$ такие, что

$$
L\left(x \cdot t_{x}\right)=c+\varepsilon, \quad L\left(x \cdot \tau_{x}\right)=c-\varepsilon
$$

и $x \cdot t$ содержится в $W$ для любого $t \in\left(t_{x}, \tau_{x}\right)$. Заметим, что если $x \in C$, то $x \cdot\left(t_{x}, \tau_{x}\right)$ содержится в $C$.

Определим следующее множество:

$$
B=C \cup Z \cup \bigcup_{0<\varepsilon^{\prime}<\varepsilon}\left(A_{+}\left(\varepsilon^{\prime}\right) \cup A_{-}\left(\varepsilon^{\prime}\right)\right) .
$$

Докажем, что оно удовлетворяет условиям теоремы 8.1. Это множество открыто. Действительно, составляющие в формуле (44) попарно не пересекаются. Очевидно, что множество $C$ открыто. У каждой точки множества $A_{ \pm}\left(\varepsilon^{\prime}\right)$ (при $0<\varepsilon^{\prime}<\varepsilon$ ) есть окрестность, целиком входящая в $B$ (согласно леммам 8.6 и 8.7). Аналогично, любая достаточно малая окрестность $G$ точки $x \in Z$ содержится в $B$ : если $y \in G$ и $L(y)>c$, то траектория $y \cdot t$ при $t>0$ либо приближается к $Z$ (в этом случае $y$ лежит в $A_{+}\left(\varepsilon^{\prime}\right)$ для некоторого $0<\varepsilon^{\prime}<\varepsilon$ ), либо наталкивается на поверхность уровня $L=c$. Во втором случае точка пересечения находится очень близко от $Z$ и, следовательно, по леммам 8.6 и 8.7 траектория на всем своем продолжении остается в $W$, пока не достигнет уровня $L=c-\varepsilon$.

Для данного $x \in M$ рассмотрим множество $J_{x}=\{t ; x \cdot t \in B\}$. Предположим, что $L(x)>c$. Имеются следующие возможности:

1) $\ell(x)>c$, тогда $J_{x}=\varnothing$;

2) $\omega(x) \subset J_{x}$, тогда $J_{x}$ является полубесконечным интервалом $(a,+\infty)$;

3) $\ell(x)=c$ и $\omega(x) \cap Z=\varnothing$, тогда $J_{x}=\varnothing$;

4) $\ell(x)<c$, тогда $J_{x}$ - либо пустое множество, либо конечный интервал $(a, b)$.

В случае $L(x) \leqslant c$ рассуждения аналогичны.

Непрерывность отображения $x \mapsto \inf J_{x}$ следует из теоремы о неявной функции: число $\inf J_{x}=t$ является решением уравнения $L(x \cdot t)=c+\varepsilon$ и частная производная функции $L(x \cdot t)$ по $t$ строго отрицательна. Теорема 8.1 доказана. 


\section{9. Доказательство теоремы 7.6}

Рассуждения аналогичны тем, что использовались в доказательстве теоремы 4.1 в [15].

Предположим, что теорема 7.6 неверна, т. е. для любого $N>0$ существует градиентоподобное векторное поле $v=v_{N}$ формы $\omega$, у которого нет обобщенных гомоклинических циклов, состоящих из интегральных траекторий $\gamma_{1}, \ldots, \gamma_{\ell}$, удовлетворяющих (39) и (40). Наша цель - показать, что тогда

$$
\operatorname{cat}(M,[\omega]) \leqslant \sum_{i=1}^{k} \operatorname{cat}_{M}\left(Y_{i}\right)
$$

т. е. неравенства (38) нарушаются. При $k=\infty$ неравенство (45) очевидно, поскольку $\operatorname{cat}_{M}\left(Y_{i}\right) \geqslant 1$ для любого $i$; поэтому без потери общности можно предположить, что число компонент связности $k$ конечно.

Зададим риманову метрику на $M$. Поток $M \times \mathbb{R} \rightarrow M$, порожденный полем $-v$, обозначим через $t \mapsto m \cdot t$, где $m \in M$ и $t \in \mathbb{R}$. Поскольку $\omega(v)>0$ на $M-Y$, интеграл $\int_{p}^{p \cdot t} \omega \leqslant 0$ неположительный и не возрастающий при $t>0$.

Начнем с доказательства следующей леммы.

Лемма 9.1. Пусть $N>0$ - такое иелое число, что существует градиентоподобное векторное поле $v$ для $\omega$, не имеющее обобщенных гомоклинических ииклов, состоящих из интегралъных траекторий $\gamma_{1}, \ldots, \gamma_{\ell}$, удовлетворяюших (39) и (40). Пусть $U_{i}, Y_{i} \subset U_{i} \subset M$, - открытая окрестность $Y_{i}$, $i=1, \ldots, k$. Тогда существует семейство менъших замкнутых окрестностей $V_{i} \subset U_{i}$ множества $Y_{i}$, обладающих следующими свойствами.

(а) Каждое множество $V_{i}$ поднимается до накрывающей $\pi: \widetilde{M} \rightarrow M$, соответствующей ядру гомоморфизма периодов $\pi_{1}(M) \rightarrow \mathbb{R}$, определяемого классом когомологий $\xi=[\omega] \in H^{1}(M ; \mathbb{R})$.

(b) Поднятие $V_{i}$ в $\widetilde{M}$ является выпуклым относительно потока (т.е. удовлетворяет свойствам (А) и (В) из теоремы 8.1); на накрывающей $\widetilde{M}$ рассматривается поток $\tilde{v}$, явяющиися естественным поднятием поmoкa $v$.

(c) Пусть д_ $V_{i}-$ множество выхода для $V_{i}$, m.е. множество всех точек $p \in V_{i}$ таких, что для достаточно малых $\tau>0$ имеем $p \cdot \tau \notin V_{i}$. Тогда не существует $p \in \partial_{-} V_{i} u t>0$ mаких, что $p \cdot t \in \operatorname{Int} V_{i} u \int_{p}^{p \cdot t} \omega \geqslant-N$.

Интуитивно свойство (с) означает, что траектория не может покинуть $V_{i}$ и вернуться туда же за время $t>0$ без того, чтобы величина $\int_{p}^{p \cdot t} \omega$ стала
меньше $-N$ (ср. рис. 11).

ДокАзАТЕЛЬСтво. Без ограничения общности можно считать, что начальные окрестности $U_{i}$ обладают свойствами (а) и (b).

Пусть $V_{i} \subset U_{i}$ - произвольная открытая окрестность $Y_{i}$ со свойством (b); тогда (а) выполнено автоматически. Обозначим через $V, U$ и $\partial_{-} V$ следующие объединения: $V=V_{1} \cup \cdots \cup V_{k}, U=U_{1} \cup \cdots \cup U_{k}$ и $\partial_{-} V=\partial_{-} V_{1} \cup \cdots \cup \partial_{-} V_{k}$. 

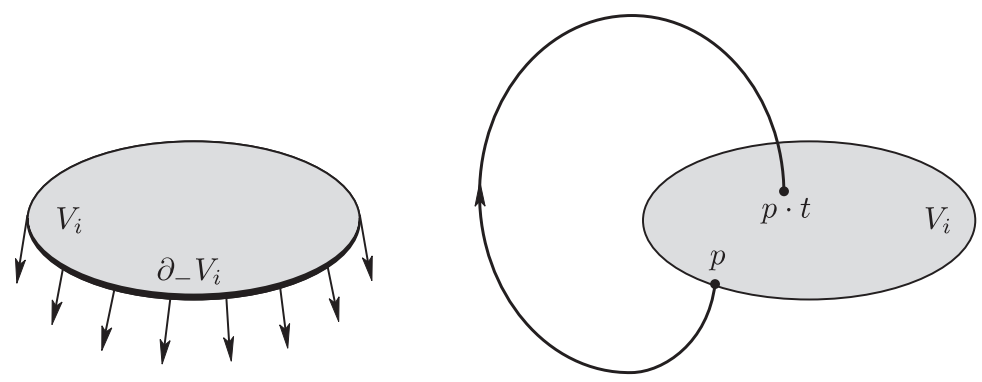

Рис. 11

Мы утверждаем, что

(i) существует такое $a>0$, что для любъх $p \in \partial_{-} V u t>0$ таких, что $p \cdot t \in V$, имеет место неравенство $t \geqslant a$.

Действительно, как следует из градиентной выпуклости множеств $U_{i}$, любая траектория $p \cdot t$, начинающаяся в точке $p \in \partial_{-} V$ при $t=0$, покинет $U$, прежде чем сможет вернуться в $V$. Следовательно, мы можем взять $a=\min \left\{l_{i} v_{i}^{-1}\right.$; $i=1, \ldots, k\}$, где $l_{i}>0$ обозначает расстояние между $V_{i}$ и $M-\operatorname{Int} U_{i}$, и $v_{i}=$ $\max |v(x)|$ для $x \in U_{i}-\operatorname{Int} V_{i}$.

Заметим, что при сжатии множеств $V_{i}$ число $a>0$ может только увеличиться в предположении, что $V_{i}$ достаточно малы.

(ii) Cуществует такое $b>0$, что для любых $p \in \partial_{-} V u t>0$ maких, что $p \cdot t \in V$, имеет место неравенство

$$
\int_{p}^{p \cdot t} \omega<-b
$$

Действительно, можно взять $b=a \cdot \min \{\omega(v(x)) ; x \in U-V\}$.

Из (i) и (ii) следует, что

(iii) существует такое целое $K>0$, что для любого $p \in \partial_{-} V$ множество

$$
I(p)=\left\{t>0 ; p \cdot t \in V u \int_{p}^{p \cdot t} \omega \geqslant-N\right\}
$$

является объединением не более $K$ непересекающихся интервалов, т.е.

$$
I(p)=\bigcup_{s=1}^{k(p)}\left[a_{s}(p), b_{s}(p)\right], \quad k(p) \leqslant K,
$$

$u$

$$
0<a_{s}(p) \leqslant b_{s}(p)<a_{s+1}(p) .
$$

Действительно, количество “дырок" между интервалами $\left[a_{s}(p), b_{s}(p)\right]$ не больше чем $[N / b]$, как это следует из (ii).

Для точки $q \in M$ обозначим через

$$
\mathscr{A}(q)=\bigcap_{t \in \mathbb{R}} \overline{q \cdot(-\infty, t]}, \quad \mathscr{Z}(q)=\bigcap_{t \in \mathbb{R}} \overline{q \cdot[t, \infty)},
$$


прямое и обратное предельные множества траекторий, проходящих через $q$. Эти множества непусты, компактны, связны и инвариантны относительно потока.

Предположим, что невозможно добиться выполнения (с) сжатием начальных окрестностей $V_{i} \supset Y_{i}$. Мы хотим показать, что тогда существуют последовательность точек $q_{1}, \ldots, q_{\ell} \in M$ и вложение $j:\{1, \ldots, \ell\} \rightarrow\{1, \ldots, k\}$, для которых выполнены включения

$$
\mathscr{A}\left(q_{i}\right) \subset Y_{j(i)}, \quad \mathscr{Z}\left(q_{i}\right) \subset Y_{j(i+1)},
$$

где $i=1, \ldots, \ell$, и $j(\ell+1)=j(1)$ и неравенство

$$
\sum_{i=1}^{\ell} \int_{\gamma_{i}} \omega \geqslant-N,
$$

где кривая $\gamma_{i}: \mathbb{R} \rightarrow M$ задана как $\gamma_{i}(t)=q_{i} \cdot t$ при $t \in \mathbb{R}$, что будет противоречить нашим предположениям.

Существуют бесконечная последовательность точек $p_{n} \in \partial_{-} V, n=1,2, \ldots$, и две последовательности вещественных чисел $t_{n}>0$ и $s_{n}<0$ такие, что

1) множество $p_{n} \cdot\left[s_{n}, 0\right]$ содержится в выбранной компоненте связности $V_{i} \subset V$ и расстояние $d\left(p_{n} \cdot s_{n}, Y_{i}\right)$ стремится $\kappa$ нулю при $n \rightarrow \infty$;

2) $d\left(p_{n} \cdot t_{n}, Y_{i}\right)$ сходится $\kappa$ нулю при $n \rightarrow \infty$;

3) выполнено неравенство

$$
\int_{p_{n}}^{p_{n} \cdot t_{n}} \omega \geqslant-N .
$$

Переходя к подпоследовательности, мы можем предположить, что $p_{n}$ сходится к точке $p \in \partial_{-} V_{i}$ и последовательности $s_{n}$ и $t_{n}$ имеют конечные или бесконечные пределы, которые мы обозначим соответственно $s$ и $t$. Ввиду свойства (iii), мы также можем предположить, что количество $\kappa\left(p_{n}\right)=\kappa$ интервалов не зависит от $n$ и последовательности $a_{s}\left(p_{n}\right)$ и $b_{s}\left(p_{n}\right)$ имеют конечные или бесконечные пределы $a_{s}$ и $b_{s}$ соответственно, $s=1, \ldots, \kappa$. Более того, из тех же соображений можно предположить, что при $n \rightarrow \infty$ последовательности

$$
p_{n} \cdot a_{s}\left(p_{n}\right) \in V \quad \text { и } \quad p_{n} \cdot b_{s}\left(p_{n}\right) \in V
$$

сходятся к точкам $q_{s}, q_{s}^{\prime} \in V$ соответственно, где $s=1, \ldots, \kappa$.

Легко видеть, что из 1) и 2) следует $t=+\infty$ и $s=-\infty$.

Заметим, что $\mathscr{A}(p) \subset Y_{i}$. Действительно, согласно 1$)$, имеем $p \cdot(-\infty, 0] \subset V_{i}$, и поэтому множество $\mathscr{A}(p)$ должно содержаться в максимальном инвариантном подмножестве $Y_{i}$ в $V_{i}$. Очевидно, что точки $q_{s}, q_{s}^{\prime}$ лежат на границе одной и той же компоненты связности $V_{j_{s}}$ множества $V$, где $s \mapsto j_{s}$ отображает $\{1, \ldots, \kappa\}$ в $\{1, \ldots, k\}$ и $j_{\kappa}=i$ (ср. рис. 12). Как следует из приведенных выше аргументов, точки $q_{s}$ и $q_{s}^{\prime}$ либо лежат на одной орбите потока, либо имеют место включения

$$
\mathscr{Z}\left(q_{s}\right) \subset Y_{j_{s}}, \quad \mathscr{A}\left(q_{s}^{\prime}\right) \subset Y_{j_{s}} .
$$

Первый случай можно не рассматривать, поскольку тогда надо просто поменять метки точек и применить следующие наблюдения. Точки $q_{s}^{\prime}$ и $q_{s+1}$ принадлежат одной орбите и поэтому (49) можно переписать в следующем виде:

$$
\mathscr{Z}\left(q_{s}\right) \subset Y_{j_{s}}, \quad \mathscr{A}\left(q_{s+1}\right) \subset Y_{j_{s}},
$$




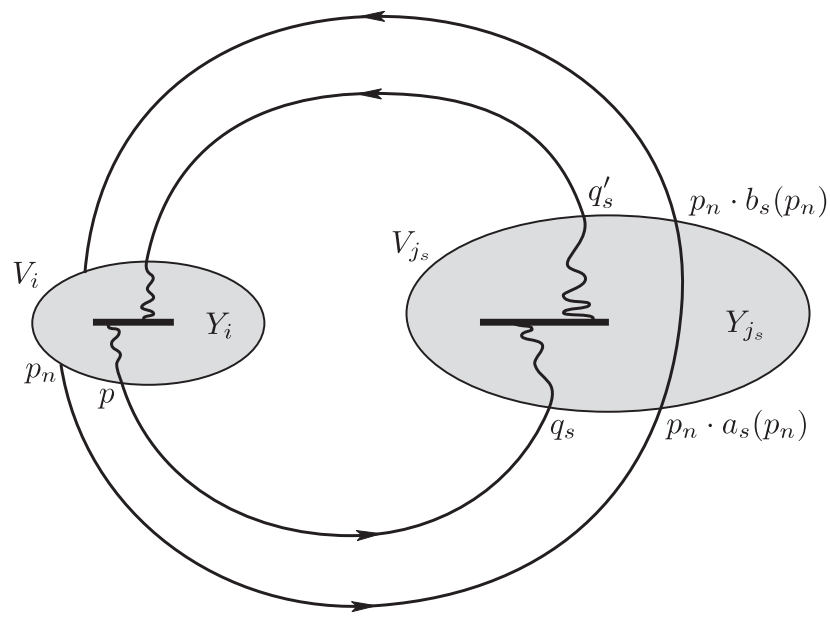

Рис. 12

где $s=1, \ldots, \kappa-1$. Для последней точки $q_{\kappa}$ имеем

$$
\mathscr{Z}\left(q_{\kappa}\right) \subset Y_{i} \quad \text { и } \quad \mathscr{A}\left(q_{1}\right)=\mathscr{A}(p) \subset Y_{i} .
$$

Следовательно, получаем замкнутую цепочку интегральных траекторий обобщенный гомоклинический цикл. Неравенство (47) получается из (48), если перейти к пределу и заметить, что форма $\left.\omega\right|_{V_{j}}$ является точной: $\left.\omega\right|_{V_{j}}=d f_{j}$, где $f_{j}: V_{j} \rightarrow \mathbb{R}$ - гладкая функция. Более того, функция $f_{j}$ постоянна на $Y_{j}$. Действительно, образ $f_{j}\left(Y_{j}\right) \subset \mathbb{R}$ является связным и по теореме Сарда имеет меру нуль; поэтому $f_{j}\left(Y_{j}\right)$ состоит из одной точки. Из этих замечаний следует, что

$$
\lim _{n \rightarrow \infty} \int_{p_{n} \cdot a_{s}\left(p_{n}\right)}^{p_{n} \cdot b_{s}\left(p_{n}\right)} \omega=\lim _{T \rightarrow \infty} \int_{q_{s}}^{q_{s} \cdot T} \omega+\lim _{S \rightarrow-\infty} \int_{q_{s}^{\prime} \cdot S}^{q_{s}^{\prime}} \omega
$$

для любого $s=1, \ldots, \kappa$.

Таким образом, лемма 9.1 доказана.

ДОКАЗАТЕЛЬСТвО ТЕОРЕМЫ 7.6. Предположим, что при условиях теоремы 7.6 не существует обобщенных гомоклинических циклов, для которых выполнено неравенство (40). Используя лемму 9.1 с окрестностями $W_{i}$ такими, что $\operatorname{cat}_{M}\left(W_{i}\right)=\operatorname{cat}\left(Y_{i}\right)$, получим систему окрестностей $Y_{i} \subset V_{i}$, удовлетворяющих условиям (а)-(с) леммы 9.1 , а также условиям $\operatorname{cat}_{M}\left(V_{i}\right)=\operatorname{cat}_{M}\left(Y_{i}\right)$, $i=1, \ldots, k$.

Определим множества $F_{1}, \ldots, F_{k} \subset M$ следующим образом. Будем говорить, что $p \in F_{i}$, если при некотором $t_{p}>0$ точка $p \cdot t_{p}$ принадлежит внутренности $V_{i}$ и

$$
\int_{p}^{p \cdot t_{p}} \omega>-N
$$

Очевидно, что множество $F_{i}$ является открытым.

Покажем, что множество $F_{i} \subset M$ можно продеформировать в $V_{i} \subset F_{i}$. Введем для любой точки $p \in M$ множество $J_{p} \subset \mathbb{R}$ следующим образом: 
$J_{p}=\left\{t \geqslant 0 ; p \cdot t \in V_{i}\right\}$. В силу предположения градиентной выпуклости окрестности $V_{i}$, множество $J_{p}$ является объединением непересекающихся замкнутых интервалов, некоторые из этих интервалов могут вырождаться в точки. Рассмотрим первый интервал $\left[\alpha_{p}, \beta_{p}\right] \subset J_{p}$. Если он вырождается в точку (т. е. траектория, проходящая через $p$, касается $V_{i}$ ), то, согласно предположению (c), точка $p$ не принадлежит множеству $F_{i}$; см. выше. По тем же соображениям точки из $\partial_{-} V_{i}$ не принадлежат $F_{i}$.

Предположим, что $p \in F_{i}$ и $p \notin \operatorname{Int} V_{i}$. Тогда точка $p \cdot t$ лежит во внутренности множества $V_{i}$ при $\alpha_{p}<t<\beta_{p}$. Кроме того, имеем

$$
\int_{p}^{p \cdot \alpha_{p}} \omega>-N
$$

Функция $\varphi_{i}: F_{i} \rightarrow \mathbb{R}$, заданная формулой

$$
\varphi_{i}(p)= \begin{cases}0, & \text { если } p \in \operatorname{Int} V_{i}, \\ \alpha_{p}, & \text { если } p \in F_{i}-\operatorname{Int} V_{i},\end{cases}
$$

непрерывна, поскольку $\left(\partial_{-} V_{i}\right) \cap F_{i}=\varnothing$. Можно задать следующую гомотопию:

$$
h_{\tau}^{i}: F_{i} \rightarrow M, \quad h_{\tau}^{i}(p)=p \cdot\left(\tau \varphi_{i}(p)\right), \quad p \in F_{i}, \quad \tau \in[0,1] .
$$

Здесь $h_{0}^{i}$ - вложение $F_{i} \rightarrow M$, а $h_{1}^{i}$ отображает $F_{i}$ во внутренность множества $V_{i}$. По лемме 19.5 из [37] получаем

$$
\operatorname{cat}_{M}\left(F_{i}\right) \leqslant \operatorname{cat}_{M}\left(V_{i}\right)=\operatorname{cat}_{M}\left(Y_{i}\right)
$$

и, следовательно,

$$
\operatorname{cat}_{M}\left(\bigcup_{i=1}^{k} F_{i}\right) \leqslant \sum_{i=1}^{k} \operatorname{cat}_{M}\left(Y_{i}\right) .
$$

Рассмотрим теперь дополнение $A=M-\bigcup_{i=1}^{k} F_{i}$. Для любой точки $p \in A$ существует $t_{p}>0$ такое, что

$$
\int_{p}^{p \cdot t_{p}} \omega=-N
$$

Очевидно, что отображение $p \mapsto t_{p}$ является непрерывной вещественнозначной функцией на $A$. Можно задать гомотопию

$$
h_{\tau}: A \rightarrow A
$$

формулой

$$
h_{\tau}(p)=p \cdot\left(\tau t_{p}\right), \quad \tau \in[0,1] .
$$

Тогда для любого $x \in A$ имеем

$$
\int_{x}^{h_{1}(x)} \omega=-N
$$


т. е. замкнутое множество $A$ является $N$-подвижным относительно $\omega$ и

$$
\operatorname{cat}_{M}(M-A) \leqslant \sum_{i=1}^{k} \operatorname{cat}_{M}\left(Y_{i}\right) .
$$

Согласно определению 6.2 , получаем

$$
\operatorname{cat}(M,[\omega]) \leqslant \sum_{i=1}^{k} \operatorname{cat}_{M}\left(Y_{i}\right),
$$

что завершает доказательство теоремы 7.6.

\section{0. Топология цепно-рекуррентного множества $R_{\xi}$}

В этом разделе мы докажем следующий результат из [51].

Теорема 10.1 (Фарбер-Каппелер). Рассмотрим гладкий поток $\Phi: M \times$ $\mathbb{R} \rightarrow M$ на замкнутом гладком многообразии $M$. Пусть $\xi \in H^{1}(M ; \mathbb{R})-$ класс когомологий, удовлетворяющий следующим условиям:

1) цепно-рекуррентное множество $R_{\xi}$ изолировано в полном иепно-рекуррентном множестве $R$ потока $\Phi$;

2) ограничение

$$
\left.\xi\right|_{R_{\xi}} \in \check{H}^{1}\left(R_{\xi} ; \mathbb{R}\right)
$$

(рассмотренное как класс когомологий Чеха) равно нулю;

3) для любой $\Phi$-инвариантной положительной меры Бореля $\mu$ на $M$ такой, что $\mu(R)>\mu\left(R_{\xi}\right)$, асимптотический иикл Шваримана

$$
\mathscr{A}_{\mu}=\mathscr{A}_{\mu}(\Phi) \in H_{1}(M ; \mathbb{R})
$$

удовлетворяет неравенству

$$
\left\langle\xi, \mathscr{A}_{\mu}\right\rangle<0
$$

Тогда верно следующее неравенство:

$$
\sum_{i=1}^{r} \operatorname{cat}_{M}\left(R_{\xi}^{i}\right) \geqslant \operatorname{cat}_{\mathrm{s}}^{1}(M, \xi),
$$

где $R_{\xi}^{1}, \ldots, R_{\xi}^{r}$ - компоненты связности ${ }^{5}$ иепно-рекуррентного множества $R_{\xi}$.

Доказательство теоремы 10.1 дано в разделе 11.

Сопоставим теорему 10.1 с теоремой 6.6 из [16]. Теорема 10.1 допускает произвольные множества $R_{\xi}$, тогда как в теореме 6.6 работы [16] предполагается, что $R_{\xi}$ состоит из конечного числа изолированных точек. Другое важное достоинство теоремы 10.1 в том, что она не требует выполнения условия (*) из [16; с. 108], которое сложно проверять в конкретных примерах. С другой стороны, поскольку $\operatorname{cat}_{s}^{1}(X, \xi) \leqslant \operatorname{Cat}(X, \xi)$, то оценка из теоремы $6.6[16]$ является потенциально чуть более сильной.

\footnotetext{
5число $r$ компонент связности множества $R_{\xi}$ может быть бесконечным. В этом случае неравенство (14) выполняется тривиально, поскольку его левая часть бесконечна.
} 
Сформулируем некоторые следствия из теоремы 10.1.

В частном случае $\xi=0$ имеем $R_{\xi}=R$ и предположения 1$\left.)-3\right)$ теоремы 10.1 выполнены автоматически. Таким образом, получаем следующий результат, который, по-видимому, хорошо известен, но который нам не удалось найти в литературе.

Теорема 10.2. Рассмотрим гладкий поток на замкнутом гладком многообразии $M$. Пусть $R$ - иепно-рекуррентное множество этого потока. Тогда

$$
\sum_{i=1}^{r} \operatorname{cat}_{M}\left(R^{i}\right) \geqslant \operatorname{cat}(M),
$$

где $R^{1}, \ldots, R^{r}$ компоненты связности $R$.

В частном случае, когда цепно-рекуррентное множество $R$ состоит из конечного числа точек (неподвижные точки потока), теорема 10.2 утверждает, что количество неподвижных точек не меньше категории Люстерника-Шнирелъмана многообразия $M$. Это один из фундаментальных результатов классической теории Люстерника-Шнирельмана (см. [39]).

Рассмотрим другой частный случай теоремы 10.1 , когда $R_{\xi}$ состоит из конечного числа точек (они являются неподвижными точками потока). При этих предположениях условие 1) теоремы 10.1 выполнено автоматически. Получаем следующее утверждение.

Теорема 10.3 (Фабер-Каппелер). Рассмотрим гладкий поток на замкнутом гладком многообразии $M$. Пусть $\xi \in H^{1}(M ; \mathbb{R})$ - класс когомологий такой, что цепно-рекуррентное множество $R_{\xi}$ состоит из конечного числа точек, изолированных в полном иепно-рекуррентном множестве $R$ потока. Кроме того, предположим, что выполнено условие 3) теоремы 10.1. Тогда число неподвижных точек потока не менъше $\operatorname{cat}_{\mathrm{s}}^{1}(M, \xi)$.

Приведем переформулировку теоремы 10.3 .

Теорема 10.4 (Фарбер-Каппелер). Пусть $\xi \in H^{1}(M ; \mathbb{R})$ - комологический класс. Рассмотрим поток $\Phi$ на $M$, иепно-рекуррентное множество $R_{\xi}$ которого состоит из менее чем $\operatorname{cat}_{s}^{1}(M, \xi)$ неподвижных точек и для которого выполнено условие 3) теоремы 10.1. Тогда хотя бы одна неподвижная точка не является изолированной в полном цепно-рекуррентном множестве $R$.

Приведем типичное применение теоремы 10.3 .

Теорема 10.5 (Фарбер-Каппелер). Пусть на замкнутом гладком многообразии задан гладкий поток $\Phi$, цепно-рекуррентное множество $R$ которого является обгединением конечного числа окружностей и изолированных точек. Пусть $\xi \in H^{1}(M ; \mathbb{R})$ - класс когомологий такой, что $\langle\xi, z\rangle \leqslant 0$ для гомологического класса $z \in H_{1}(M)$ любой периодической орбитъ. Тогда

$$
p_{0}+p_{1}+2 p_{2} \geqslant \operatorname{cat}_{\mathrm{s}}^{1}(M, \xi),
$$

где $p_{0}$ - количество неподвижных точек потока, $p_{1}$ - количество стягиваемых в $M$ периодических орбит, а р 2 - количество гомотопически нетривиальных орбит, гомологические классы $z \in H_{1}(M)$ которых удовлетворяют $\langle\xi, z\rangle=0$. 
ДокАЗАТЕЛЬСтво. В частном случае теоремы 10.5 условие 3) теоремы 10.1 эквивалентно требованию $\langle\xi, z\rangle \leqslant 0$ для гомологического класса $z \in H_{1}(M)$ любой периодической орбиты. В предположениях теоремы 10.5 множество $R_{\xi}$ является объединением неподвижных точек и периодических орбит, удовлетворяющих $\langle\xi, z\rangle=0$. Если $C$ - периодическая орбита, то число $\operatorname{cat}_{M}(C)$ равно единице или двум в зависимости от того, стягивается $C$ в $M$ или нет. Это рассуждение показывает, что теорема 10.5 следует из теоремы 10.1.

Заметим попутно, что в условиях теоремы 10.5

$$
p_{0}+p_{1}+2 p_{2}+2 q_{2} \geqslant \operatorname{cat}(M),
$$

как следует из теоремы 10.2 ; здесь $q_{2}$ - количество периодических орбит, удовлетворяющих условию $\langle\xi, z\rangle \neq 0$.

\section{1. Доказательство теоремы 10.1}

Рассмотрим гладкий поток $\Phi: M \times \mathbb{R} \rightarrow M, \Phi(x, t)=x \cdot t$, и вещественный класс когомологий $\xi \in H^{1}(M ; \mathbb{R})$, удовлетворяющие условиям теоремы 10.1 .

Если цепно-рекуррентное множество $R_{\xi}$ состоит из бесконечного числа компонент связности, то правая часть неравенства (52) бесконечна и оно становится очевидным. Следовательно, без ограничения общности можно считать, что число компонент связности множества $R_{\xi}$ конечно.

Пусть $R_{\xi}^{1}, \ldots, R_{\xi}^{r}$ - компоненты связности множества $R_{\xi}, R_{\xi}=R_{\xi}^{1} \cup \cdots \cup R_{\xi}^{r}$. Зафиксируем непересекающиеся открытые окрестности $W_{i} \supset R_{\xi}^{i}, i=1, \ldots, r$. Предположим, что множества $W_{i}$ настолько малы, что

$$
\operatorname{cat}_{M}\left(W_{i}\right)=\operatorname{cat}_{M}\left(R_{\xi}^{i}\right), \quad i=1, \ldots, r
$$

(ср. [39; леммы 19.2 и 19.6]).

Применяя теорему 8.1, можно найти выпуклую относительно потока $\Phi$ открытую окрестность $B_{i} \subset W_{i}$ множества $R_{\xi}^{i}$. Очевидно, что вследствие $(56)$ имеют место равенства

$$
\operatorname{cat}_{M}\left(\bar{B}_{i}\right)=\operatorname{cat}_{M}\left(R_{\xi}^{i}\right), \quad i=1, \ldots, r .
$$

Пусть $U_{i}$ - открытая окрестность $R_{\xi}^{i}$, замыкание $\bar{U}_{i}$ которой содержится в $B_{i}$. Имеют место следующие включения: $R_{\xi}^{i} \subset U_{i} \subset \bar{U}_{i} \subset B_{i} \subset W_{i}$.

Пусть $A \subset M$ - множество точек $x \in M$ таких, что для любого $t \in \mathbb{R}$ точка $x \cdot t$ не принадлежит объединению $U=\bigcup_{j=1}^{r} U_{j}$. Множество $A$ является замкнутым и инвариантным относительно потока.

Используя теорему 5.5 , заключаем, что для пары $\left(\Phi, R_{\xi}\right)$ существует гладкая 1-форма Ляпунова $\omega$, задающая класс когомологий $\xi=[\omega] \in H^{1}(M ; \mathbb{R})$. Это означает, что функция $\omega(V): M \rightarrow \mathbb{R}$ отрицательна на $M-R_{\xi}$ (где $V-$ векторное поле на $M$, порожденное потоком $\Phi)$ и $\left.\omega(V)\right|_{R_{\xi}}=0$. Более того, ограничение 1-формы $\omega$ на некоторую открытую окрестность $R_{\xi}$ является дифференциалом гладкой функции (это эквивалентно условию 1) теоремы 10.1).

Покажем, что для любого целого $N$ множество $A$ является $N$-подвижным как относительно $\omega$, так и относительно $-\omega$. Поскольку $M-U$ компактно, то 
существует $\varepsilon>0$ такое, что $\omega(V)<-\varepsilon$ на $M-U$. Тогда для любого $x \in A$ имеем

$$
\int_{x}^{x \cdot t} \omega<-\varepsilon t \quad \text { при } t>0 .
$$

Следовательно, непрерывная гомотопия $h_{t}: A \rightarrow M, t \in[0,1]$, определенная формулой

$$
h_{t}(x)=x \cdot\left(\frac{N t}{\varepsilon}\right),
$$

удовлетворяет условию определения 6.1. Это показывает, что множество $A$ является $N$-подвижным относительно $\omega$. Похожим образом можно показать, что для любого целого $N$ множество $A$ является $N$-подвижным относительно $-\omega$.

Теперь для доказательства неравенства (52) достаточно показать, что

$$
\operatorname{cat}_{M}(M-A) \leqslant \sum_{j=1}^{r} \operatorname{cat}_{M}\left(R_{\xi}^{j}\right)
$$

(см. определение 6.3).

Пусть $F_{i} \subset M$ - множество таких точек $x \in M$, что $x \cdot t$ принадлежит $B_{i}$ для некоторого $t \in \mathbb{R}$. Тогда множество $J_{x}^{i}=\left\{t \in \mathbb{R} ; x \cdot t \in B_{i}\right\}$ является непустым открытым интервалом. Для $x \in F_{i}$ определим

$$
\tau_{i}(x)= \begin{cases}0, & \text { если } 0 \in J_{x}^{i}, \\ \inf J_{x}^{i}, & \text { если } J_{x}^{i} \subset(0, \infty), \\ \sup J_{x}^{i}, & \text { если } J_{x}^{i} \subset(-\infty, 0) .\end{cases}
$$

Как следует из теоремы 8.1, функция $\tau_{i}: F_{i} \rightarrow \mathbb{R}$ непрерывна.

Отображение $x \mapsto x \cdot \tau_{i}(x)$ является деформационной ретракцией $F_{i} \rightarrow \bar{B}_{i}$ и, следовательно,

$$
\operatorname{cat}_{M}\left(F_{i}\right) \leqslant \operatorname{cat}_{M}\left(\bar{B}_{i}\right)=\operatorname{cat}_{M}\left(R_{\xi}^{i}\right)
$$

ввиду (57). Здесь мы использовали лемму 19.5 из [39]. Поскольку $M-A$ содержится в объединении $\bigcup_{i=1}^{r} F_{i}$, получаем

$$
\operatorname{cat}_{M}(M-A) \leqslant \sum_{i=1}^{r} \operatorname{cat}_{M}\left(F_{i}\right) \leqslant \sum_{i=1}^{r} \operatorname{cat}_{M}\left(R_{\xi}^{i}\right) .
$$

Тем самым доказано неравенство (58) и, следовательно, завершено доказательство теоремы 10.1.

\section{2. Когомологические оценки $\operatorname{cat}(X, \xi)$}

Эффективность динамических приложений, описанных в разделах 7-11, существенно зависит от возможности вычисления или оценки снизу величины $\operatorname{cat}(X, \xi)$ для конечного полиэдра $X$ и когомологического класса $\xi \in H^{1}(X ; \mathbb{R})$. Ситуация очень похожа на классическую теорию Люстерника-Шнирельмана, где $\operatorname{cat}(X)$ чаще всего вычисляется комбинацией верхних и нижних оценок и наиболее популярной нижней оценкой является когомологическая длина (см. [39]). 
В этом разделе мы обсудим подобные проблемы для $\operatorname{cat}(X, \xi)$, следуя нашей работе [55]; некоторые нижние оценки для $\operatorname{cat}(X, \xi)$ были получены ранее в работах [15] и [13]. В этом разделе мы дадим основные определения, сформулируем наиболее важные результаты и проиллюстрируем их несколькими примерами; однако для полного доказательства мы отсылаем читателя к нашим оригинальным работам [55], [56] и [57].

Пусть $X$ - конечный полиэдр и $\xi \in H^{1}(X ; \mathbb{R})$ - когомологический класс. Обозначим через $\operatorname{Ker}(\xi)$ ядро гомоморфизма $\pi_{1}(X) \rightarrow \mathbb{R}$, заданного "интегрированием" класса $\xi$. Тогда $H=\pi_{1}(X) / \operatorname{Ker}(\xi)$ - свободная абелева группа ранга $r$, где $r$ - степень иррациональности $\xi$. Рассмотрим накрытие $p: \widetilde{X} \rightarrow X$, соответствующее ядру $\operatorname{Ker}(\xi)$. Группа $H$ является группой монодромии этого накрытия.

Пусть $\mathscr{V}_{\xi}=\left(\mathbb{C}^{*}\right)^{r}=\operatorname{Hom}\left(H, \mathbb{C}^{*}\right)$ - многообразие комплексных, плоских одномерных векторных расслоений $L$ над $X$ таких, что индуцированное плоское одномерное векторное расслоение $p^{*} L$ над $\widetilde{X}$ является тривиальным. Если $t_{1}, \ldots, t_{r} \in H-$ базис, то монодромия $L \in \mathscr{V}_{\xi}$ вдоль $t_{i}$ является ненулевым комплексным числом $x_{i} \in \mathbb{C}^{*}$ и значения $x_{1}, \ldots, x_{r} \in \mathbb{C}^{*}$ задают систему координат на $\mathscr{V}_{\xi}$. Для данного плоского одномерного векторного расслоения $L \in \mathscr{V}_{\xi}$ представление монодромии для $L$ является кольцевым гомоморфизмом

$$
\operatorname{Mon}_{L}: \mathbb{C}[H] \rightarrow \mathbb{C}
$$

переводящим $t_{i} \in H$ в $x_{i} \in \mathbb{C}^{*}$. Для двойственного расслоения $L^{*} \in \mathscr{V}_{\xi}$ произведение $L \otimes L^{*}$ тривиально; если $x_{1}, \ldots, x_{r} \in \mathbb{C}^{*}-$ координаты в $L$, то $x_{1}^{-1}, \ldots, x_{r}^{-1} \in \mathbb{C}^{*}$ являются координатами в $L^{*}$.

Любой нетривиальный элемент $p \in \mathbb{C}[H]$ из ядра гомоморфизма $\operatorname{Mon}_{L}$ можно рассматривать как полиномиальное (в смысле Лорана) соотношение между переменными $x_{1}, \ldots, x_{r}$. Другими словами, мы будем рассматривать как алгебраические подмногообразия $V \subset \mathscr{V}_{\xi}$. Каждое такое $V$ является множеством всех решений системы уравнений вида

$$
q_{i}\left(x_{1}, \ldots, x_{r}, x_{1}^{-1}, \ldots, x_{r}^{-1}\right)=0, \quad i=1, \ldots, m,
$$

где $q_{i}$ - лорановские полиномы с комплексными коэффициентами

$$
p_{i} \in \mathbb{C}\left[x_{1}, \ldots, x_{r}, x_{1}^{-1}, \ldots, x_{r}^{-1}\right] .
$$

Это равносильно фиксации идеала $J \subset \mathbb{Q}[H]$ и изучению множества таких плоских одномерных векторных расслоений $L \in \mathscr{V}_{\xi}$, для которых $\operatorname{Mon}_{L}(J)=0$.

ОПРедЕЛЕНиЕ 12.1. 1) Расслоение $L \in \mathscr{V}_{\xi}$ будем называть $\xi$-алгебраичес$\kappa u м$, если гомоморфизм монодромии $\operatorname{Mon}_{L}: \mathbb{Z}[H] \rightarrow \mathbb{C}$ имеет нетривиальное ядро.

2) Расслоение $L \in \mathscr{V}_{\xi}$ будем называть $\xi$-алгебраически целым, если ядро гомоморфизма монодромии $\operatorname{Mon}_{L}: \mathbb{Z}[H] \rightarrow \mathbb{C}$ содержит ненулевые многочлены $P \in \mathbb{Z}[H]$ с $\xi$-старшим коэффициентом, равным 1 (см. ниже).

3) Расслоение $L$ называется $\xi$-трансцендентным, если $\operatorname{Mon}_{L}: \mathbb{Z}[H] \rightarrow \mathbb{C}$ инъективен. 
Любой ненулевой $P \in \mathbb{Z}[H]$ можно записать в виде $P=\sum_{i=1}^{k} \alpha_{i} h_{i}$, где $\alpha_{i} \in \mathbb{Z}$, $\alpha_{i} \neq 0, h_{i} \in H$ и $\xi\left(h_{1}\right)<\xi\left(h_{2}\right)<\cdots<\xi\left(h_{k}\right)$. Ненулевое целое число $\alpha_{k} \in \mathbb{Z}$ называется $\xi$-старшим коэфбичиентом $P$.

Если $t_{1}, \ldots, t_{r} \in H$ - базис и $a_{i} \in \mathbb{C}$ - монодромия расслоения $L$ вдоль $t_{i}$, т. е. $a_{i}=\operatorname{Mon}_{L}\left(t_{i}\right)$, то $L$ является $\xi$-алгебраическим тогда и только тогда, когда существует нетривиальный лорановский полином $q\left(t_{1}, \ldots, t_{r}\right)$ с целыми коэффициентами, для которого $q\left(a_{1}, \ldots, a_{r}\right)=0$.

Существует счетное множество ненулевых лорановских полиномов $q$ с целыми коэффициентами, и для любого такого $q$ множество решений уравнения $q\left(a_{1}, \ldots, a_{r}\right)=0$ нигде не плотно в $\mathscr{V}_{\xi}$. Поскольку $\mathscr{V}_{\xi}=\left(\mathbb{C}^{*}\right)^{r}$ гомеоморфно полному метрическому пространству, получаем следующий результат.

ЛЕмма 12.2. Множество всех $\xi$-трансцендентных расслоений $L \in \mathscr{V}_{\xi}$ является множеством бэровской категории 2. В частности, множество $\xi$-трансцендентных расслоений $L \in \mathscr{V}_{\xi}$ является плотным в многообразии $\mathscr{V}_{\xi}$.

ЛЕмма 12.3. Для любого $\xi$-трансцендентного плоского одномерного расслоения $L \in \mathscr{V}_{\xi}$ размерность векторного пространства $H^{q}(X ; L)$ равна q-мерному числу Новикова-Бетти $b_{q}(\xi)$ :

$$
\operatorname{dim} H^{q}(X ; L)=b_{q}(\xi) .
$$

ДокАЗАТЕЛЬСтво. Гомоморфизм монодромии $\operatorname{Mon}_{L}: \mathbb{Z}[H] \rightarrow \mathbb{C}$ задает структуру левого $\mathbb{Z}[H]$-модуля $\mathbb{C}_{L}$ на пространстве $\mathbb{C}$ и по определению

$$
H^{q}(X ; L)=H^{q}\left(\operatorname{Hom}_{\mathbb{Z}[H]}\left(C_{*}(\widetilde{X}), \mathbb{C}_{L}\right)\right),
$$

где $C_{*}(\tilde{X})$ является клеточным цепным комплексом для накрытия $\widetilde{X} \rightarrow X$, соответствующего ядру $\operatorname{Ker}(\xi)$. Если $L$ трансцендентно, то Mon $_{L}$ задает расширение поля $Q(H) \rightarrow \mathbb{C}$, где $Q(H)$ - поле частных для $\mathbb{Z}[H]$. Следовательно, получаем (используя конечномерность $C_{*}(\widetilde{X})$ над $\mathbb{Z}[H]$ и $(60)$ ):

$$
\begin{aligned}
H^{q}(X ; L) & \simeq H^{q}\left(\operatorname{Hom}_{\mathbb{Z}[H]}\left(C_{*}(\tilde{X}) ; Q(H)\right)\right) \otimes_{Q(H)} \mathbb{C}_{L} \\
& \simeq H^{q}(X ; Q(H)) \otimes_{Q(H)} \mathbb{C}_{L} .
\end{aligned}
$$

Отсюда следует, что для любого трансцендентного $L$

$$
\operatorname{dim}_{\mathbb{C}} H^{q}(X ; L)=\operatorname{dim}_{Q(H)} H^{q}(X ; Q(H)) .
$$

Наконец, используем предложение 1.30 из [13], которое объясняет, почему правая часть равенства (61) равна числу Новикова-Бетти $b_{q}(\xi)$.

Теорема 12.4 (Фарбер-Шютц [55]). Пусть $X$ - конечный клеточный комплекс, $\xi \in H^{1}(X ; \mathbb{R})$ - вещественный одномерный класс когомологий $u L \in \mathscr{V}_{\xi}$ есть $\xi$-трансцендентное расслоение. Предположим, что существуют когомологические классы $v_{0} \in H^{d_{0}}(X ; L)$ u $v_{i} \in H^{d_{i}}(X ; \mathbb{C}), i=1, \ldots, k$, для которьх $d_{i}>0$ при $i \in\{1, \ldots, k\}$ и произведение которых не равно нулю:

$$
v_{0} \cup v_{1} \cup \cdots \cup v_{k} \neq 0 \in H^{*}(X ; L) .
$$

Тогда $\operatorname{cat}(X, \xi)>k$.

Теорема 12.4 сочетает в себе простоту и исключительную эффективность. Далее в настоящем разделе мы будем применять эту теорему во многих специфических примерах. 
12.1. Понятие когомологической длины $\operatorname{cl}(X, \xi)$. Принимая во внимание теорему 12.4, введем следующее понятие.

Пусть $X$ - конечный клеточный комплекс и $\xi \in H^{1}(X ; \mathbb{R})$ - одномерный класс когомологий. Обозначим $\operatorname{cl}(X, \xi)$ максимальное целое число $k \geqslant 0$ такое, что для пары $(X, \xi)$ применима теорема 12.4 ; если теорему 12.4 применять нельзя (т. е. если $H^{*}(X ; L)=0$ для любого $\xi$-трансцендентного расслоения $L \in \mathscr{V}_{\xi}$, ср. с леммой 12.3), положим

$$
\operatorname{cl}(X, \xi)=-1
$$

Тогда

$$
\operatorname{cl}(X, \xi) \in\{-1,0,1, \ldots\}
$$

Другими словами, $\operatorname{cl}(X, \xi) \geqslant k$, где $k \geqslant 0$, тогда и только тогда, когда существуют $\xi$-трансцендентное плоское одномерное расслоение $L \in \mathscr{V}_{\xi}$ и когомологические классы $v_{0} \in H^{d_{0}}(X ; L)$ и $v_{i} \in H^{d_{i}}(X ; \mathbb{C})$, где $i=1, \ldots, k$ и $d_{i}>0$ при $i \in\{1, \ldots, k\}$, для которых произведение

$$
v_{0} \cup v_{1} \cup \cdots \cup v_{k} \neq 0 \in H^{*}(X ; L)
$$

нетривиально.

Заметим, что при $\xi=0$ число $\operatorname{cl}(X, \xi)$ совпадает с обычной когомологической длиной $\mathrm{cl}(X)$; напомним, что последняя определяется как максимальное целое $r$, для которого существуют когомологические классы $u_{i} \in H^{d_{i}}(X ; \mathbb{C})$, $i=1, \ldots, r$, положительных градуировок такие, что их произведение нетривиально: $u_{1} \cup \cdots \cup u_{k} \neq 0 \in H^{*}(X ; L)$.

Теорему 12.4 можно переформулировать следующим образом:

ТЕОРема 12.5. Для любого конечного клеточного комплекса X и класса когомологий $\xi \in H^{1}(X ; \mathbb{R})$ имеет место неравенство

$$
\operatorname{cat}(X, \xi) \geqslant \operatorname{cl}(X, \xi)+1
$$

Следующая полезная лемма дает несколько различных способов описания числа $\operatorname{cl}(X, \xi)$. Она будет играть важную роль в дальнейшем.

Лемма 12.6. Пусть $X$ - конечный клеточный комплекс и $\xi \in H^{1}(X ; \mathbb{R})$. Тогда следующие утверждения для целого числа $k \geqslant 0$ равносилъны:

(A) $\operatorname{cl}(X, \xi) \geqslant k$;

(В) существуют когомологический класс $v_{0} \in H^{d_{0}}(X ; L)$, где $L \in \mathscr{V}_{\xi}$ $\xi$-трансцендентное плоское одномерное расслоение, $и k$ целочисленных когомологических классов $v_{i} \in H^{d_{i}}(X ; \mathbb{Z})$, где $i=1, \ldots, k u d_{i}>0$ nри $i \in\{1, \ldots, k\}$, для которых произведение (64) нетривиально;

(C) пусть $H$ - бакторгруппа $H_{1}(X ; \mathbb{Z}) / \operatorname{Ker}(\xi)$ и $Q(H)$ - поле частных группового кольща $\mathbb{Z}[H] ;$ тогда существуют когомологические классъ $w_{0} \in$ $H^{d_{0}}(X ; Q(H))$ u $v_{i} \in H^{d_{i}}(X ; \mathbb{Z})$, где $d_{i}>0$ при $i \in\{1, \ldots, k\}$, maкие, что произведение

$$
w_{0} \cup v_{1} \cup \cdots \cup v_{k} \neq 0 \in H^{*}(X ; Q(H))
$$

не равно нулю; 
(D) для любого $\xi$-трансцендентного, плоского, одномерного векторного расслоения $L \in \mathscr{V}_{\xi}$ существует класс когомологий $v_{0} \in H^{d_{0}}(X ; L)$ такой, что произведение (64) нетривиально для некоторых иелочисленных когомологических классов $v_{i} \in H^{d_{i}}(X ; \mathbb{Z})$, где $d_{i}>0$ при $i \in\{1, \ldots, k\}$;

(Е) для любого $\xi$-трансцендентного, плоского, одномерного векторного расслоения $L \in \mathscr{V}_{\xi}$ существуют когомологические классы $v_{0} \in H^{d_{0}}(X ; L)$ u $v_{i} \in H^{d_{i}}(X ; \mathbb{C})$, где $i=1, \ldots, k u d_{i}>0$ при $i \in\{1, \ldots, k\}$, произведение (64) которых нетривиально.

ДоказАтельство. Докажем импликацию $(\mathrm{A}) \Rightarrow(\mathrm{B})$. Выберем $\xi$-трансцендентное расслоение $L \in \mathscr{V}_{\xi}$ и $v_{0} \in H^{*}(X ; L)$ такие, что произведение (64) нетривиально для некоторых $v_{i} \in H^{d_{i}}(X ; \mathbb{C})$, где $d_{i}>0$. Для произвольных целочисленных когомологических классов $v_{i}^{\prime} \in H^{d_{i}}(X ; \mathbb{Z})$ рассмотрим произведение

$$
v_{0} \cup v_{1}^{\prime} \cup \cdots \cup v_{k}^{\prime}
$$

градуировки $d_{i}$ предполагаем фиксированными. Формула (65) задает полилинейную функцию от классов $v_{i}^{\prime}$. Поскольку целочисленные классы порождают $H^{d_{i}}(X ; \mathbb{C})$ над $\mathbb{C}$, получаем, что произведение $(65)$ должно быть ненулевым при некотором выборе классов $v_{i}^{\prime}$, т. е. имеет место (B).

Теперь покажем, что $(\mathrm{B}) \Rightarrow(\mathrm{C})$. Зафиксируем $L \in \mathscr{V}_{\xi}$ и когомологические классы $v_{0} \in H^{d_{0}}(X ; L)$ и $v_{i} \in H^{d_{i}}(X ; \mathbb{Z})$, удовлетворяющие условиям пункта $(\mathrm{B})$. Гомоморфизм монодромии $\operatorname{Mon}_{L}: \mathbb{Z}[H] \rightarrow \mathbb{C}$ является инъективным кольцевым гомоморфизмом, и его можно продолжить до гомоморфизма поля частных $Q(H) \rightarrow \mathbb{C}$. Образ индуцированного гомоморфизма в когомологиях

$$
\psi: H^{d_{0}}(X ; Q(H)) \rightarrow H^{d_{0}}(X ; L)
$$

порождает $H^{d_{0}}(X ; L)$ над $\mathbb{C}$, а сам гомоморфизм (66) (по соображениям, приведенным в доказательстве леммы 12.3) является инъективным. Зафиксируем когомологические классы $v_{i} \in H^{*}(X ; \mathbb{Z}), i=1, \ldots, k$. Для класса когомологий $w_{0} \in H^{d_{0}}(X ; Q(H))$ функция

$$
\psi\left(w_{0}\right) \cup v_{1} \cup \cdots \cup v_{k}=\psi\left(w_{0} \cup v_{1} \cup \cdots \cup v_{k}\right) \in H^{*}(X ; L)
$$

продолжается до $\mathbb{C}$-линейной функции от $v_{0} \in H^{d_{0}}(X ; L)$ :

$$
v_{0} \mapsto v_{0} \cup v_{1} \cup \cdots \cup v_{k} \in H^{*}(X ; L) .
$$

Если эта функция ненулевая, то она не может обращаться в нуль на образе функции $\psi$, т. е. имеет место (C).

Импликация $(\mathrm{C}) \Rightarrow(\mathrm{D})$ следует из инъективности гомоморфизма (66) и равенства (67).

Импликации $(\mathrm{D}) \Rightarrow(\mathrm{E})$ и $(\mathrm{E}) \Rightarrow(\mathrm{A})$ очевидны. Доказательство завершено.

ЛЕмма 12.7. Предположим, что $X$ и $Y$ - линейно связные конечные клеточные комплексы и $\xi \in H^{1}(X \times Y ; \mathbb{R})$. Тогда

$$
\operatorname{cl}(X \times Y, \xi) \geqslant \operatorname{cl}\left(X,\left.\xi\right|_{X}\right)+\operatorname{cl}\left(Y,\left.\xi\right|_{Y}\right) .
$$


ДоказАтельство. Обозначим $\operatorname{cl}\left(X,\left.\xi\right|_{X}\right)=k$ и $\mathrm{cl}\left(Y,\left.\xi\right|_{Y}\right)=r$. Любое плоское одномерное векторное расслоение $L$ над $X \times Y$ имеет вид $L_{1} \otimes L_{2}$ (внешнее тензорное произведение), где $L_{1}$ и $L_{2}$ - плоские одномерные векторные расслоения над $X$ и $Y$ соответственно. Заметим, что если $L$ принадлежит многообразию $\mathscr{V}_{\xi}=\operatorname{Hom}\left(H_{1}(X \times Y ; \mathbb{Z}) / \operatorname{Ker}(\xi), \mathbb{C}^{*}\right)$, то $L_{1}$ и $L_{2}$ получаются ограничениями и, следовательно, $L_{1} \in \mathscr{V}_{\left.\xi\right|_{X}}$ и $L_{2} \in \mathscr{V}_{\left.\xi\right|_{Y}}$. Используем эквивалентность пунктов (А) и (Е) из леммы 12.6. Зафиксируем $\xi$-трансцендентное расслоение $L=L_{1} \otimes L_{2} \in \mathscr{V}_{\xi}$ над $X \times Y$. Тогда оба расслоения $L_{1}$ и $L_{2}$ являются $\xi$-трансцендентными. Найдем такие классы $v_{0} \in H^{*}\left(X ; L_{1}\right), v_{1}, \ldots, v_{k} \in$ $H^{*}(X ; \mathbb{C}), \quad u_{0} \in H^{*}\left(Y ; L_{2}\right), u_{1}, \ldots, u_{r} \in H^{*}(Y ; \mathbb{C})$, для которых $v_{0} \cup v_{1} \cup$ $\cdots \cup v_{k} \neq 0$ и $u_{0} \cup u_{1} \cup \cdots \cup u_{r} \neq 0$. Имеем классы когомологий $v_{0} \times u_{0} \in$ $H^{*}(X \times Y ; L)$ и $v_{i} \times 1,1 \times u_{j} \in H^{*}(X \times Y ; \mathbb{C})$, и произведение

$$
\left(v_{0} \times u_{0}\right) \cup \prod_{i=1}^{k}\left(v_{i} \times 1\right) \cup \prod_{j=1}^{r}\left(1 \times u_{j}\right) \neq 0 \in H^{*}(X \times Y ; L)
$$

ненулевое. Здесь мы использовали формулу Кюнета

$$
H^{*}\left(X \times Y ; L_{1} \otimes L_{2}\right) \simeq H^{*}\left(X ; L_{1}\right) \otimes H^{*}\left(Y ; L_{2}\right)
$$

Это доказывает неравенство (68).

12.2. Некоторые примеры. В этом пункте применяем теорему 12.4 в некоторых простых примерах.

12.2.1. Сначала рассмотрим случай $\xi=0$. Известно, что при $\xi=0$ число $\operatorname{cat}(X, \xi)$ совпадает с классической категорией Люстерника-Шнирельмана $\operatorname{cat}(X)$ (см. [13; пример 10.8]). Посмотрим, что дает теорема 12.4 в этом случае. Многообразие $\mathscr{V}_{\xi}$ состоит из единственной точки - тривиального плоского одномерного векторного расслоения $\mathbb{C}$ над $X$. Носитель $\operatorname{Supp}(X, \xi)=\varnothing$ при $\xi=0$ всегда есть пустое множество. Следовательно, используя теорему 12.4 , можем взять $v_{0}=1 \in H^{0}(X ; \mathbb{C})$. Мы видим, что частном случае $\xi=0$ теорема 12.4 утверждает следующее: если существуют такие классы когомологий $v_{1}, \ldots, v_{k} \in H^{>0}(X ; \mathbb{C})$, что $v_{1} \cup \cdots \cup v_{k} \neq 0$, то cat $(X)>k$. Это есть классическая оценка категории Люстерника-Шнирельмана cat $(X)$ через когомологическую длину.

12.2.2. Заметим, что если $\xi \neq 0$ и $X$ связно, то $H^{0}(X ; L)=0$ для любого $L \in \mathscr{V}_{\xi}$. Заметим также, что тривиальное плоское одномерное векторное расслоение $\mathbb{C} \in \mathscr{V}_{r}$ никогда не является $\xi$-трансцендентным. Поэтому в случае $\xi \neq 0$ градуировка класса $v_{0}$ (который возникает в теореме 12.4 ) должна быть положительной. Следовательно, при $\xi \neq 0$ число $k$ из теоремы 12.4 удовлетворяет неравенству $k \leqslant \operatorname{dim} X-1$. Это рассуждение объясняет, почему при $\xi \neq 0$ теорема 12.4 не дает оценку $\operatorname{cat}(X, \xi) \geqslant \operatorname{dim} X+1$.

В предположении, что пространство $X$ связно и $\xi \neq 0$, лемма 6.9 дает

$$
\operatorname{cat}(X, \xi) \leqslant \operatorname{cat}(X)-1 \leqslant \operatorname{dim} X
$$

Это согласуется с замечанием из предыдущего абзаца. 
12.2.3. Следующий пример показывает, что в (70) может достигаться равенство, т. е. возможно, что $\operatorname{cat}(X, \xi)=\operatorname{dim} X$. Рассмотрим букет $X=Y \vee S^{1}$, где $Y$ - конечный полиэдр, и предположим, что класс когомологий $\xi \in H^{1}(X ; \mathbb{R})$ удовлетворяет соотношениям $\left.\xi\right|_{Y}=0$ и $\left.\xi\right|_{S^{1}} \neq 0$. Мы знаем, что в этом случае

$$
\operatorname{cat}(X, \xi)=\operatorname{cat}(Y)-1
$$

(см. [13; пример 10.11]).

Используем теорему 12.4. В рассматриваемом случае многообразие $\mathscr{V}_{\xi}$ совпадает с множеством $\mathbb{C}^{*}=\mathbb{C}-\{0\}$ и носитель $\operatorname{Supp}(X, \xi)$ содержит только тривиальное одномерное расслоение. Расслоение $L \in \mathscr{V}_{\xi}$ является $\xi$-трансцендентным, если монодромия вдоль окружности $S^{1}$ является $\xi$-трансцендентным комплексным числом. Для любого $L \in \mathscr{V}_{\xi}$ ограничение $\left.L\right|_{Y}$ тривиально и гомоморфизм ограничения $H^{i}(X ; L) \rightarrow H^{i}(Y ; \mathbb{C})$ сюръективен.

Предположим, что когомологическая длина $Y$ с комплексными коэффициентами равна $\ell$, т. е. существуют когомологические классы положительной градуировки $u_{0}, u_{1}, \ldots, u_{\ell-1} \in H^{>0}(Y ; \mathbb{C})$, произведение которых не равно нулю: $u_{0} \cup \cdots \cup u_{\ell-1} \neq 0$. По вышеприведенному замечанию для нетривиального $L \in \mathscr{V}_{\xi}$ получаем классы когомологий $v_{0} \in H^{*}(X ; L)$ и $v_{1}, \ldots, v_{\ell-1} \in H^{>0}(X ; \mathbb{C})$ такие, что $\left.v_{i}\right|_{Y}=u_{i}$. Поэтому $v_{0} \cup v_{1} \cup \cdots \cup v_{\ell-1} \neq 0 \in H^{*}(X ; L)$. Из теоремы 12.4 получаем $\operatorname{cat}(X, \xi)>\ell-1$, что равносильно (ввиду (71)) неравенству $\operatorname{cat}(Y)>\ell$. Последнее неравенство является классической оценкой обычной категории через когомологическую длину.

12.2.4. Теперь рассмотрим более специальный пример: $X=T^{2} \vee S^{1}$. В этом случае $H^{1}(X ; \mathbb{R})=\mathbb{R}^{3}$, и мы опишем $\operatorname{cat}(X, \xi)$ как функцию от $\xi \in$ $H^{1}(X ; \mathbb{R})=\mathbb{R}^{3}$. Обозначим через $\ell \subset \mathbb{R}^{3}$ подмножество всех классов $\xi$ таких, что $\left.\xi\right|_{T^{2}}=0$. Очевидно, что $\ell$ есть прямая, проходящая через начало системы координат в $\mathbb{R}^{3}$. Мы утверждаем, что

$$
\operatorname{cat}(X, \xi)= \begin{cases}1, & \text { если } \xi \in \mathbb{R}^{3}-\ell, \\ 2, & \text { если } \xi \in \ell-\{0\}, \\ 3, & \text { если } \xi=0 .\end{cases}
$$

Действительно, рассмотрим первый случай $\xi \notin \ell$, т. е. $\left.\xi\right|_{T^{2}} \neq 0$. Покажем, что $\operatorname{cat}(X, \xi) \leqslant 1$. Пусть $p=T^{2} \cap S^{1}$, а $q \in S^{1}$ - точка, отличная от $p$. Положим $F=X-\{q\}$ и $F_{1}=S^{1}-\{p\}$. Тогда $F \cup F_{1}=X$ является открытым покрытием пространства $X$ и $F_{1} \rightarrow X$ стягиваемо, а множество $F$ является $N$-подвижным в $X$ для любого $N$ (в предположении, что $\left.\xi\right|_{T^{2}} \neq 0$ ); это следует из гомотопической инвариантности $\operatorname{cat}(X, \xi)$ и примера 6.8 .

Поскольку по теореме 6.12 из $\operatorname{cat}(X, \xi)=0$ следовало бы $\chi(X)=0$, получаем, что $\operatorname{cat}(X, \xi)>0$ для любого $\xi$ (так как $\chi(X)=-1 \neq 0)$. Тем самым доказана первая строчка в $(72)$.

При $\xi \in \ell-\{0\}$, применяя (71), получим $\operatorname{cat}(X, \xi)=\operatorname{cat}\left(T^{2}\right)-1=2$.

При $\xi=0$ легко найти, что $\operatorname{cat}(X, \xi)=\operatorname{cat}(X)=3$. 


\section{3. Верхние оценки для $\operatorname{cat}(X, \xi)$ \\ и связь с инвариантами Бьери-Неймана-Штребеля}

В работе [26] Бьери, Нейман и Штребель ввели геометрический инвариант дискретных групп $G$, который дает информацию о конечной порожденности ядер абелевых факторов группы $G$. В этом разделе мы опишем связь между этим инвариантом и свойствами категории $\operatorname{cat}(X, \xi)$.

Напомним определение. Мы всегда будем предполагать, что группа $G$ конечно представимая, поскольку этого для наших целей достаточно. Обозначим через $S(G)$ пространство $(\operatorname{Hom}(G, \mathbb{R})-\{0\}) / \mathbb{R}_{+}$, где $\mathbb{R}_{+}$действует на $\operatorname{Hom}(G, \mathbb{R})$ умножениями. Очевидно, что $S(G)$ является сферой размерности $n-1$, где $n-$ ранг абелизации группы $G$. Пусть $[\chi]$ обозначает класс эквивалентности ненулевого гомоморфизма $\chi: G \rightarrow \mathbb{R}$. Инвариант Бьери-Неймана-Штребеля ставит в соответствие группе $G$ подмножество $\Sigma(G) \subset S(G)$, определенное следующим образом ${ }^{6}$. Пусть $X$ - конечный клеточный комплекс с фундаментальной группой $G$ и $p: \widetilde{X} \rightarrow X-$ универсальное абелево накрытие $X$. Гомоморфизм $\chi \in \operatorname{Hom}(G, \mathbb{R})$ можно рассматривать как класс когомологий из $H^{1}(X ; \mathbb{R})$. Включение $\chi \in \Sigma(G)$ выполнено тогда и только тогда, когда вложение $N \rightarrow \widetilde{X}$ индуцирует эпиморфизм $\pi_{1}\left(N, x_{0}\right) \rightarrow \pi_{1}\left(\widetilde{X}, x_{0}\right)$, где $N \subset \widetilde{X}-$ связная окрестность бесконечности относительно $\chi$ (см. [55; § 2] и [26; лемма 5.2].

Следующая теорема дает сводку результатов, доказанных в [55].

ТЕОРема 13.1 (Фарбер-Шютц). (а) Пусть $X$ - конечный связный полиэдр и $\xi \in H^{1}(X ; \mathbb{R})$ - ненулевой класс когомологий. Тогда

$$
\operatorname{cat}(X, \xi) \leqslant \operatorname{dim} X
$$

(b) Пусть $M$ - замкнутое, связное, гладкое многообразие и $\xi \in H^{1}(M ; \mathbb{R})$ ненулевой класс. Тогда

$$
\operatorname{cat}(M, \xi) \leqslant \operatorname{dim}(M)-1 \text {. }
$$

(c) Пусть $M-$ замкнутое, связное, гладкое многообразие размерности $\geqslant 5$ и $\xi \in H^{1}(M ; \mathbb{R})$ - ненулевой класс. Если

$$
\xi \in \Sigma\left(\pi_{1}(M)\right) \quad \text { или }-\xi \in \Sigma\left(\pi_{1}(M)\right),
$$

mo

$$
\operatorname{cat}(M, \xi) \leqslant \operatorname{dim} M-2 .
$$

(d) Пусть $M$ - замкнутое, связное, гладкое многообразие размерности $\geqslant 5$ и $\xi \in H^{1}(M ; \mathbb{R})$ - ненулевой класс. Если имеют место оба включения

$$
\xi \in \Sigma\left(\pi_{1}(M)\right) \quad u \quad-\xi \in \Sigma\left(\pi_{1}(M)\right),
$$

mo

$$
\operatorname{cat}(M, \xi) \leqslant \operatorname{dim} M-3 .
$$

\footnotetext{
${ }^{6}$ Мы полагаемся на теорему 5.1 работы [26], которая утверждает, что определение инварианта $\Sigma(G)$, приведенное выше, конечно представимой группы $G$ совпадает с основным определение из работы [26].
} 
14. Гомологические категорные веса, оценки для $\operatorname{cat}^{1}(X, \xi)$ и вычисление $\operatorname{cat}(X, \xi), \operatorname{cat}^{1}(X, \xi)$ для произведений поверхностей

В этом разделе мы кратко опишем результаты работы [56], основной целью которых является получение когомологических нижних оценок для $\operatorname{cat}^{1}(X, \xi)$. В частности, мы увидим, что есть много примеров, когда $\operatorname{cat}(X, \xi)<\operatorname{cat}^{1}(X, \xi)$ и разность $\operatorname{cat}^{1}(X, \xi)-\operatorname{cat}(X, \xi)$ может быть сколь угодно большой.

Первым шагом является введение нового понятия категорного веса гомологических классов, которое в некотором смысле двойственно когомологическому понятию, введенному Фаделлом и Хуссейни [58]. Приятной неожиданностью стало то, что гомологический категорный вес, в отличие от когомологической версии Фаделла и Хуссейни, оказался гомотопическим инвариантом.

Понятие гомологического категорного веса позволило нам получить улучшенную нижнюю когомологическую оценку для $\operatorname{cat}^{1}(X ; \xi)$.

14.1. Гомологический категорный вес. Классическая когомологическая нижняя оценка для категории Люстерника-Шнирельмана $\operatorname{cat}(X)$ устанавливает, что $\operatorname{cat}(X)>n$, если существует $n$ классов когомологий $u_{j} \in$ $H^{*}\left(X ; R_{j}\right), j=1,2, \ldots, n$, положительной градуировки, произведение которых нетривиально:

$$
u_{1} u_{2} \cdots u_{n} \neq 0 \in H^{*}(X ; R) .
$$

Здесь $R_{j}$ обозначает локальную систему коэффициентов на $X$, а $R$ - тензорное произведение $R=R_{1} \otimes \cdots \otimes R_{n}$.

Фаделл и Хуссейни [58] улучшили эту оценку, введя новое понятие категорного веса $\operatorname{cwgt}(u)$ для когомологического класса $u \in H^{q}(X ; R)$.

ОПРЕДЕЛЕНИЕ 14.1 (Фаделл-Хуссейни, [58]). Пусть $u \in H^{q}(X ; R)$ - ненулевой класс когомологий, где $R$ - система локальных коэффициентов на $X$. Будем говорить, что $\operatorname{cwgt}(u) \geqslant k$ (где $k \geqslant 0$ - целое число), если для любого замкнутого подмножества $A \subset X$ такого, что $\operatorname{cat}_{X} A \leqslant k$, верно $\left.u\right|_{A}=0 \in$ $H^{q}(A ; R)$.

Напомним, что $\operatorname{cat}_{X} A \leqslant k$ означает, что $A$ может быть покрыто $k$ открытыми подмножествами $U_{i} \subset X, i=1, \ldots, k$, каждое из которых стягиваемо в $X$.

Согласно определению 14.1, имеем $\operatorname{cwgt}(u) \geqslant 0$ в общем случае и $\operatorname{cwgt}(u) \geqslant 1$ для любого ненулевого когомологического класса положительной градуировки. Как показано в [58], в некоторых специальных ситуациях $\operatorname{cwgt}(u)>1$, что улучшает нижнюю оценку для $\operatorname{cat}(X)$. Действительно, имеем

$$
\operatorname{cat}(X) \geqslant 1+\sum_{i=1}^{n} \operatorname{cwgt}\left(u_{i}\right)
$$

в предположении, что произведение $u_{1} u_{2} \cdots u_{n}$ ненулевое: $u_{1} u_{2} \cdots u_{n} \neq 0$.

Рудяк [59] и Стром [60] изучали модификацию категорного веса $\operatorname{cwgt}(u)$, называемую строгим категорным весом $\operatorname{swgt}(u)$. Последний имеет то преимущество, что он является гомотопическим инвариантом. Однако в некоторых примерах строгий категорный вес значительно меньше, чем первоначальный категорный вес Фаделла и Хуссейни. 
В работе [56] мы ввели и использовали “двойственное" понятие категорного веса гомологических классов. Оно обладает геометрической простотой и ясностью категорного веса Фаделла и Хуссейни и имеет то удивительное преимущество, что является гомотопическим инвариантом.

ОПРЕДЕЛЕниЕ 14.2 (Фарбер-Шютц, [56]). Пусть $z \in H_{q}(X ; R)$ - сингулярный гомологический класс с коэффициентами в локальной системе $R$ и $k \geqslant 0-$ неотрицательное целое число. Будем говорить, что $\operatorname{cwgt}(z) \geqslant k$, если для любого замкнутого подмножества $A \subset X$ такого, что саt $X A \leqslant k$, существует сингулярный цикл $c$ в $X-A$, представляющий $z$. Будем говорить, что $\operatorname{cwgt}(z)=k$ тогда и только тогда, когда $\operatorname{cwgt}(z) \geqslant k$ и $\operatorname{cwgt}(z) \ngtr k+1$.

Другими словами, $\operatorname{cwgt}(z) \geqslant k$ эквивалентно тому, что класс $z$ может быть реализован сингулярным циклом, не пересекающимся ни с каким замкнутым подмножеством $A \subset X$ таким, что cat $_{X} A \leqslant k$.

Например, $\operatorname{cwgt}(z) \geqslant 1$, если и только если $z$ можно реализовать сингулярным циклом, обходящим все замкнутые подмножества $A \subset X$, для которых вложение $A \rightarrow X$ стягиваемо в точку.

Нам будет удобно положить категорный вес нулевого гомологического класса равным $+\infty$.

Формально, $\operatorname{cwgt}(z) \geqslant k$, если $z$ лежит в пересечении

$$
\bigcap_{A} \operatorname{Im}\left[H_{q}(X-A ; R) \rightarrow H_{q}(X ; R)\right]
$$

где $A \subset X$ пробегает все замкнутые подмножества такие, что саt ${ }_{X} A \leqslant k$.

Соотношение $\operatorname{cwgt}(z) \leqslant k$ означает, что существует замкнутое подмножество $A \subset X$, cat $_{X} A \leqslant k+1$, для которого любая геометрическая реализация класса $z$ пересекает $A$. В частности, для любого гомологического класса $z \in H_{q}(X ; R)$, $z \neq 0$, имеем неравенство

$$
\operatorname{cat}(X) \geqslant \operatorname{cwgt}(z)+1
$$

Последнее неравенство можно переписать в следующем виде:

$$
0 \leqslant \operatorname{cwgt}(z) \leqslant \operatorname{cat}(X)-1 \leqslant \operatorname{dim} X
$$

для любого ненулевого класса гомологий.

Заметим, что если $X$ линейно связно и $z$-нульмерный класс, т. е. $z \in H_{0}(X)$, то $\operatorname{cwgt}(z)=\operatorname{cat}(X)-1$.

Лемма 14.3. Пусть $X-$ симплициалъный полиэдр. Тогда $\operatorname{cwgt}(z) \geqslant k$, если и только если z можно реализовать в $X-A$ для любого подкомплекса $A \subset X$ maкого, чmo $\operatorname{cat}_{X} A \leqslant k$.

ДокАЗАТЕЛьство. Нам надо доказать только утверждение части "если". Пусть подмножество $A \subset X$ замкнуто и cat $_{X} A \leqslant k$. Мы должны показать, что $z$ можно реализовать циклом в $X-A$. Имеем $A \subset U_{1} \cup \cdots \cup U_{k}$, где каждое $U_{i}$ открыто и стягиваемо в $X$. Переходя к более мелкому подразбиению комплекса $X$, можем найти подкомплекс $B \subset X$ такой, что $A \subset$ $B \subset U_{1} \cup \cdots \cup U_{k}$. Тогда $\operatorname{cat}_{X} B \leqslant k$ и $z$ можно реализовать циклом, принадлежащим $X-B \subset X-A$. Лемма 14.3 доказана. 
ПримеР 14.4. Пусть $X$ - замкнутое двумерное многообразие, т. е. компактная поверхность. Покажем, что для любого ненулевого класса $z \in H_{1}(X)$ выполнено неравенство $\operatorname{cwgt}(z) \geqslant 1$. Действительно, легко видеть, что любое замкнутое подмножество $A \subset X$, стягиваемое в $X$, лежит во внутренности диска $D^{2} \subset X$; но отображение $H_{1}\left(X-\operatorname{Int} D^{2}\right) \rightarrow H_{1}(X)$ является изоморфизмом.

Теорема 14.5 (Фарбер-Шютц [56]). Если $f: X \rightarrow Y$ - гомотопическая эквивалентность, то для любого класса гомологий $z \in H_{q}(X ; R)$ имеет место равенство

$$
\operatorname{cwgt}(z)=\operatorname{cwgt}\left(f_{*}(z)\right)
$$

Здесь $f_{*}(z) \in H_{q}\left(X ; R^{\prime}\right)$, где $R^{\prime}=g^{*} R$ - система локальных коэфбициентов над $Y$, индуцированная отображением $g: Y \rightarrow X$, гомотопически обратным $\kappa f$.

Следующий важный результат из [56] показывает, что понятие гомологического категорного веса в сочетании с определением Фаделла и Хуссейни можно использовать для оценивания $\operatorname{cat}(X)$.

СЛЕДСТВИЕ 14.6. Пусть $X$ - метрическое пространство и для некоторых классов $z \in H_{q}(X ; R)$ u $u \in H^{q}\left(X ; R^{\prime}\right)$ значение

$$
\langle u, z\rangle \neq 0 \in R^{\prime} \otimes R
$$

не равно нулю. Тогда

$$
\operatorname{cat}(X) \geqslant \operatorname{cwgt}(z)+\operatorname{cwgt}(u)+1 .
$$

Здесь $\operatorname{cwgt}(z)$ - категорный вес класса гомологий $z$, как он определен выше, $a \operatorname{cwgt}(u)$ - категорный вес класса и, как он определен Фаделлом и Хуссейни в [58].

Основная идея этого следствия состоит в том, что "степенъ нетривиалъности" когомологического класса и можно "измерить", с помощью "свойств", гомологического класса $z$, удовлетворяющего неравенству $\langle u, z\rangle \neq 0$.

Любопытно, что в случае замкнутых многообразий наше понятие категорного веса гомологических классов совпадает с когомологическим категорным весом Фаделла и Хуссейни по двойственности Пуанкаре. Однако для комплексов Пуанкаре это разные понятия, как показывает приводимый ниже пример.

Теорема 14.7 (Фарбер-Шютц [56]). Пусть $X$ - замкнутое $n$-мерное многообразие и $z \in H_{q}(X ; R)$, где $R$ - локальная система коэффициентов. Пусть $u \in H^{n-q}(X ; R \otimes \widetilde{\mathbb{Z}})$ - двойственный по Пуанкаре класс когомологий, т.е. $z=$ $u \cap[X]$. Тогда

$$
\operatorname{cwgt}(z)=\operatorname{cwgt}(u)
$$

Здесъ $\widetilde{\mathbb{Z}}$ обозначает ориентированную локальную систему над $X$, m.е. ростком пучка $\widetilde{\mathbb{Z}}$ в точке $x \in X$ является $\widetilde{\mathbb{Z}}_{x}=H_{n}(X, X-x ; \mathbb{Z})($ см. [46]).

ПримеР 14.8. Пусть $X=\mathbb{R P}^{n}$ - вещественное проективное пространство. Для единственного ненулевого когомологического класса $z \in H_{q}\left(X ; \mathbb{Z}_{2}\right)$ имеем $\operatorname{cwgt}(z)=n-q$. Действительно, двойственным гомологическим классом является $\alpha^{n-q} \in H^{n-q}\left(X ; \mathbb{Z}_{2}\right)$, где $\alpha \in H^{1}\left(X ; \mathbb{Z}_{2}\right)$ - образующая. Очевидно, что $\operatorname{cwgt}\left(\alpha^{n-q}\right)=n-q$. 
Из теоремы 14.7 вытекает следующее утверждение.

СлЕДСТвИЕ 14.9. Если $X$ - замкнутое $n$-мерное многообразие, то для любого класса гомологий $z \in H_{q}(X ; R), q<n$, имеем

$$
\operatorname{cwgt}(z) \geqslant 1 \text {. }
$$

Действительно, при $q<n$ двойственный класс когомологий $u$ имеет положительную градуировку и поэтому $\operatorname{cwgt}(u) \geqslant 1$.

Теперь рассмотрим случай, когда $X$ является $n$-мерным комплексом Пуанкаре. Следующий пример показывает, что теорема 14.7 для комплексов Пуанкаре не верна. Модификация рассуждений Д. Пуппе показывает, что категорный вес когомологических классов не является гомотопическим инвариантом.

ПримеР 14.10. Рассмотрим линзовое пространство $L=S^{2 n+1} /(\mathbb{Z} / p)$, где $p-$ нечетное простое число и $\mathbb{Z} / p$ действует свободно на $S^{2 n+1}$. Обозначим через $r: S^{2 n+1} \rightarrow L$ соответствующее расслоение. Пусть $X$ - цилиндр отображения $r$, т. e.

$$
X=L \sqcup S^{2 n+1} \times[0,1] / \sim,
$$

где каждая точка $(x, 0) \in S^{2 n+1} \times[0,1]$ отождествляется с $r(x) \in L$. Очевидно, что $X$ гомотопически эквивалентно $L$ и, следовательно, является комплексом Пуанкаре. Согласно теореме Красносельского [61], категория $X$ равна $2 n+2$. Следовательно, для $z=1 \in H_{0}\left(X ; \mathbb{Z}_{2}\right)$

$$
\operatorname{cwgt}(z)=\operatorname{cat}(X)-1=2 n+1
$$

(см. выше). Двойственный класс когомологий $u$ является образующей группы $H^{2 n+1}\left(X ; \mathbb{Z}_{2}\right)$. Покажем, что

$$
\operatorname{cwgt}(u)=1 \text {. }
$$

Действительно, рассмотрим сферу $S=S^{2 n+1} \times 1 \subset X$. Ограничение $\left.u\right|_{S} \in$ $H^{2 n+1}\left(S ; \mathbb{Z}_{2}\right)$ совпадает с индуцированным классом $r^{*}(v)$, где $v \in H^{2 n+1}\left(L ; \mathbb{Z}_{2}\right)$ образующая. Поэтому когомологический класс $\left.u\right|_{S}$ ненулевой. Следовательно, категория сферы $S$ равна 2 и, более того, cat $_{X} S=2$ (поскольку вложение $S \rightarrow X$ не стягиваемо).

14.2. Когомологическая оценка для $\operatorname{cat}^{1}(X, \xi)$. Следующая теорема является основным результатом работы [56].

ТеОРема 14.11 (Фарбер-Шютц [56]). Пусть $X$ - конечный клеточный комплекс и $\xi \in H^{1}(X ; \mathbb{R})$. Пусть $L \in \mathscr{V}_{\xi}$ комплексное, плоское, одномерное векторное расслоение над $X$, которое не является $\xi$-алгебраччески иелым (см. определение 12.1). Предположим, что для целочисленного класса гомологий $z \in$ $H_{q}(\widetilde{X} ; \mathbb{Z})=H_{q}(X ; \Lambda)$ и для некоторых когомологических классов $u \in H^{d}(X ; L)$ и $u_{i} \in H^{d_{i}}(X ; \mathbb{C})$, где $d_{i}>0$ nри $i=1, \ldots, k$, значения $\left\langle u \cup u_{1} \cup \cdots \cup u_{k}\right.$, $\left.p_{*}(z)\right\rangle \neq 0 \in \mathbb{C}$ не равнъ нулю. Здесъ $p_{*}(z) \in H_{d}\left(X ; L^{*}\right), q=d+d_{1}+\cdots+d_{k}$. Тогда

$$
\operatorname{cat}^{1}(X, \xi) \geqslant \operatorname{cwgt}(z)+k+1 .
$$

Здесь $\operatorname{cwgt}(z)$ обозначает категорный вес класса $z$, рассмотренного как класс гомологий комплекса $X$ с системой локальных коэффициентов $\Lambda=\mathbb{Z}[H]$, где $H=H_{1}(X ; \mathbb{Z}) / \operatorname{Ker}(\xi)$ - свободная абелева группа, которую можно отождествить с группой периодов класса $\xi$. 
14.3. Категории $\operatorname{cat}(X, \xi)$ и $\operatorname{cat}^{1}(X, \xi)$ для произведений поверхностей.

Теорема 14.12 (Фарбер-Шютц [55], [56]). Пусть $M^{2 k}$ - произведение

$$
\Sigma_{1} \times \Sigma_{2} \times \cdots \times \Sigma_{k}
$$

где каждое $\Sigma_{i}$ - замкнутая ориентированная поверхность рода $g_{i}>1$. Для заданного когомологического класса $\xi \in H^{1}\left(M^{2 k} ; \mathbb{R}\right)$ имеем

$$
\operatorname{cat}\left(M^{2 k}, \xi\right)=1+2 r
$$

$u$

$$
\operatorname{cat}^{1}\left(M^{2 k}, \xi\right)=1+k+r,
$$

где $r=r(\xi)$ обозначает число таких индексов $i \in\{1, \ldots, k\}$, что когомологический класс $\left.\xi\right|_{\Sigma_{i}} \in H^{1}\left(\Sigma_{i}, \mathbb{R}\right)$ обнуляется. В частности,

$$
\operatorname{cat}\left(M^{2 k}, \xi\right)=1
$$

$u$

$$
\operatorname{cat}^{1}\left(M^{2 k}, \xi\right)=1+k
$$

в предположении, что $\left.\xi\right|_{\Sigma_{i}} \neq 0 \in H^{1}\left(\Sigma_{i} ; \mathbb{R}\right)$ для всех $i=1, \ldots, k$.

Для доказательства и более детального ознакомления мы отсылаем читателя к нашим работам [55], [56].

Теорема 14.12 показывает, что разность между двумя понятиями категории относительно когомологического класса

$$
\operatorname{cat}^{1}(X, \xi)-\operatorname{cat}(X, \xi)
$$

может быть сколь угодно большой.

Некоторые другие результаты исследования теории Люстерника-Шнирельмана для замкнутых 1-форм были получены в [54] и [52]; см. также [16] и [51].

\section{Список литературы}

[1] С. П. Новиков, "Многозначные функции и функционалы. Аналог теории Морса", Докл. АН ССCP, 260 (1981), 31-35; англ. пер.: S. P. Novikov, "Multivalued functions and functionals. An analogue of the Morse theory", Soviet Math. Dokl., 24 (1981), 222-226.

[2] С. П. Новиков, "Гамильтонов формализм и многозначный аналог теории Морса", УМH, 37:5 (1982), 3-49; англ. пер.: S. P. Novikov, "The Hamiltonian formalism and a multi-valued analogue of Morse theory", Russian Math. Surveys, 37:5 (1982), 1-56.

[3] С. П. Новиков, "Блоховские гомологии. Критические точки функций и замкнутых 1-форм", Докл. АН СCCP, 287 (1986), 1321-1324; англ. пер.: S. P. Novikov, "Bloch homology. Critical points of functions and closed 1-forms", Soviet Math. Dokl., 33 (1986), 551-555.

[4] S. P. Novikov, "Quasiperiodic structures in topology", Topological methods in modern mathematics (Stony Brook, NY, 1991), Publish or Perish, Houston, TX, 1993, 223-235. 
[5] С. П. Новиков, "Вариационные методы и периодические решения уравнений типа Кирхгофа. II", Функи. анализ и его прил., 15:4 (1981), 37-52; англ. пер.: S. P. Novikov, "Variational methods and periodic solutions of Kirchhoff-type equations. II", Funct. Anal. Appl., 15:4 (1981), 263-274.

[6] С.П. Новиков, И. Шмельцер, "Периодические решения уравнений Кирхгофа для свободного движения твердого тела в жидкости и расширенная теория Люстерника-Шнирельмана-Морса (ЛШМ). I", Функи. анализ и его прил., 15:3 (1981), 54-66; англ. пер.: S.P. Novikov, I. Smel'tser, "Periodic solutions of the Kirchhoff's equations for the free motion of a rigid body in a fluid and the extended theory of Lyusternik-Shnirel'man-Morse theory (LSM). I", Funct. Anal. Appl., 15:3 (1981), 197-207.

[7] С. П. Новиков, И. А. Тайманов, "Периодические экстремали многозначных или не всюду положительных функционалов", Докл. АН CCCP, 274 (1984), 26-28; англ. пер.: S.P. Novikov, I. Taimanov, "Periodic extremals in multivalued or not everywhere positive functionals", Soviet Math. Dokl., 29 (1984), 18-20.

[8] J.-Cl. Sikorav, Thèse de Doctorat d'Etat Es Sciences Mathématiques, Université Paris-Sud, Centre d'Orsay, 1987.

[9] J.-Cl. Sikorav, "Un problème de disjonction par isotopie symplectique dans un fibré cotangent", Ann. Sci. École Norm. Sup. (4), 19:4 (1986), 543-552.

[10] H. Hofer, D. A. Salamon, "Floer homology and Novikov rings", The Floer memorial volume, Progr. Math., 133, Birkhäuser, Basel, 1995, 483-524.

[11] Yong-Geun Oh, "Spectral invariants, analysis of the Floer moduli space and geometry of Hamiltonian diffeomorphisms", Duke Math. J., 130:2 (2005), 199-295.

[12] M. Usher, Spectral numbers in Floer theories, arXiv: 0709.1127, 2007.

[13] M. Farber, "Topology of closed one-forms", Math. Surveys Monogr., 108, Amer. Math. Soc., Providence, RI, 2004.

[14] A. V. Pajitnov, "Circle-valued Morse theory", de Gruyter Stud. Math., 32, Walter de Gruyter, Berlin, 2006.

[15] M. Farber, "Zeros of closed 1-forms, homoclinic orbits and Lusternik-Schnirelman theory", Topol. Methods Nonlinear Anal., 19:1 (2002), 123-152.

[16] M. Farber, "Lusternik-Schnirelman theory and dynamics", Lusternik-Schnirelmann category and related topics (South Hadley, MA, 2001), Contemp. Math., 316, Amer. Math. Soc., Providence, RI, 2002, 95-111.

[17] M. Farber, T. Kappeler, J. Latschev, E. Zehnder, "Lyapunov 1-forms for flows", Ergodic Theory Dynam. Systems, 24:5 (2004), 1451-1475.

[18] M. Farber, T. Kappeler, J. Latschev, E. Zehnder, "Smooth Lyapunov 1-forms", Enseign. Math. (2), 50:1-2 (2004), 3-17.

[19] J. Latschev, "Closed forms transverse to singular foliations", Manuscripta Math., 121:3 (2006), 293-315.

[20] J. Latschev, "Coherent measures and the existence of smooth Lyapunov 1-forms for flows", Topology, 45:4 (2006), 707-723.

[21] C. Conley, "Isolated invariant sets and the Morse index", CBMS Regional Conf. Ser. in Math., 38, Amer. Math. Soc., Providence, RI, 1978.

[22] C. Conley, "The gradient structure of a flow. I", Ergodic Theory Dynam. Systems, 8 (1988), 11-26.

[23] S. Schwartzman, "Asymptotic cycles", Ann. of Math. (2), 66:2 (1957), 270-284.

[24] S. Schwartzman, "Global cross-sections of compact dynamical systems", Proc. Nat. Acad. Sci. U.S.A., 48 (1962), 786-791.

[25] M. Farber, R. Geoghegan, D. Schütz,, Closed 1-forms in topology and geometric group theory, arXiv: 0810.0962, 2008.

[26] R. Bieri, W.D. Neumann, R. Strebel, "A geometric invariant of discrete groups", Invent. Math., 90:3 (1987), 451-477. 
[27] R. Bieri, B. Renz, "Valuations on free resolutions and higher geometric invariants of groups", Comment. Math. Helv., 63:3 (1988), 464-497.

[28] А.В. Пажитнов, "О точности неравенств типа Новикова для многообразий со свободной абелевой фундаментальной группой", Матем. сб., 180:11 (1989), 1486-1523; англ. пер.: A. V. Pazhitnov, "On the sharpness of Novikov type inequalities for manifolds with free abelian fundamental group", Math. USSR Sbornik, 68 (1991), 351-389.

[29] М. Ш. Фарбер, “Точность неравенств Новикова”, Функи. анализ и его прил., 19:1 (1985), 49-59; англ. пер.: M. Sh. Farber, "Exactness of Novikov inequalities", Funct. Anal. Appl., 19:1 (1985), 40-48.

[30] M. Farber, A. Ranicki, "The Morse-Novikov theory of circle-valued functions and noncommutative localization", Солитоны, геометрия, топология - на перекрестках, Тр. МИАН, 225, Наука, М., 1999, 381-388; англ. пер.: Proc. Steklov Inst. Math., 1999, № 2, 363-371.

[31] M. Farber, "Counting zeros of closed 1-forms", Topology, ergodic theory, real algebraic geometry, Amer. Math. Soc. Transl. Ser. 2, 202, eds. V. Turaev, A. Vershik, Amer. Math. Soc., Providence, RI, 2001, 95-107.

[32] P. M. Cohn, Free rings and their relations, Second ed., London Math. Soc. Monogr., 19, Academic Press, London, 1985; пер. с англ.: П. Кон, Свободные кольца и их связи, Мир, М., 1975.

[33] M. Farber, "Morse-Novikov critical point theory, Cohn localization and Dirichlet units", Commun. Contemp. Math., 1:4 (1999), 467-495; arXiv: math/9911157, 1999.

[34] М.Ш. Фарбер, Д. Шютц, "Числа Новикова-Бетти и фундаментальная группа", УМH, 61:6 (2006), 193-194; англ. пер.: M. Farber, D. Schütz, "Novikov-Betti numbers and the fundamental group", Russian Math. Surveys, 61:6 (2006), 1173-1175.

[35] A. Pazhitnov, "Morse theory of closed 1-forms", Algebraic topology (Poznań, 1989), Lecture Notes in Math., 1474, Springer, Berlin, 1991, 98-110.

[36] A. V. Pazhitnov, "On the Novikov complex of rational Morse forms", Ann. Fac. Sci. Toulouse Math. (6), 4:2 (1995), 297-338.

[37] A. Ranicki, "Finite domination and Novikov rings", Topology, 34:3 (1995), 619-632.

[38] A. Ranicki, "The algebraic construction of the Novikov complex of a circle-valued Morse function", Math. Ann., 322:4 (2002), 745-785.

[39] Б. А. Дубровин, С. П. Новиков, А. Т. Фоменко, Современная геометрия. Методы теории гомологий, Наука, М., 1984.

[40] M. Farber, D. Schütz, "Closed 1-forms with at most one zero", Topology, 45:3 (2006), 465-473.

[41] F. Latour, "Existence de 1-formes fermées non singulières dans une classe de cohomologie de de Rham", Publ. Math. Inst. Hautes Etudes Sci., 80 (1994), 135-194.

[42] G. Levitt, "1-formes fermées singulières et groupe fondamental", Invent. Math., 88:3 (1987), 635-667.

[43] F. Takens, "The minimal number of critical points of a function on a compact manifold and the Lusternik-Schnirelman category", Invent. Math., 6:3 (1968), 197-244.

[44] A. Dold, Lectures on algebraic topology, Grundlehren Math. Wiss., 200, Springer-Verlag, New York-Berlin, 1972; пер. с англ.: Дольд А., Лекиии по алгебраической топологии, Мир, М., 1976.

[45] S. Lang, Real and functional analysis, Third ed., Grad. Texts in Math., 142, SpringerVerlag, New York, 1993.

[46] E. H. Spanier, Algebraic topology, McGraw-Hill, New York, 1966.

[47] M. Shub, Global stability of dynamical systems, Springer-Verlag, New York, 1987.

[48] D. Fried, "The geometry of cross sections to flows", Topology, 21:4 (1982), 353-371.

[49] H. Fan, J. Jost, "Novikov-Morse theory for dynamical systems", Calc. Var. Partial Differential Equations, 17:1 (2003), 29-73. 
[50] H. Fan, J. Jost, "Conley index theory and Novikov-Morse theory", Pure Appl. Math. Q., 1:4, part 3 (2005), 939-971; arXiv: math/0312018, 2003.

[51] М. Фарбер, Т. Каппелер, “Теория Люстерника-Шнирельмана и динамика. II", Геометрическая топология и теория множеств: K 100-летию со дня рожд. проф. Л.В. Келдыш: Сб. ст., Тр. МИАН, 247, 2004, 252-266; англ. пер.: M. Farber, T. Kappeler, "Lusternik-Schnirelman theory and dynamics. II", Proc. Steklov Inst. Math., 2004, № 4, 232-245.

[52] D. Schütz, "On the Lusternik-Schnirelman theory of a real cohomology class", Manuscripta Math., 113:1 (2004), 85-106.

[53] S. Smale, "Stable manifolds for differential equations and diffeomorphisms", Ann. Scuola Norm. Sup. Pisa (3), 17 (1963), 97-116.

[54] J. Latschev, "Flows with Lyapunov one-forms and a generalization of Farber's theorem on homoclinic cycles", Int. Math. Res. Not., 5 (2004), 239-247.

[55] M. Farber, D. Schütz, "Cohomological estimates for cat $(X, \xi)$ ", Geom. Topol., 11 (2007), 1255-1288.

[56] M. Farber, M. Belolipetsky, "Homological category weights and estimates for $\operatorname{cat}^{1}(X, \xi)$ ", J. Eur. Math. Soc. (JEMS), 10:1 (2008), 243-266.

[57] M. Farber, D. Schütz, "Moving homology classes to infinity", Forum Math., 19:2 (2007), 281-296.

[58] E. Fadell, S. Husseini, "Category weight and Steenrod operations", Bol. Soc. Mat. Mexicana (2), 37:1-2 (1992), 151-161.

[59] Y.B. Rudyak, "On category weight and its applications", Topology, 38:1 (1999), $37-55$.

[60] J. Strom, "Essential category weight and phantom maps", Cohomological methods in homotopy theory (Bellaterra, 1998), Progr. Math., 196, Birkhaüser, Basel, 2001, 409-415.

[61] М. А. Красносельский, "О специальных покрытиях конечномерной сферы”, Докл. AH CCCP, 103 (1955), 961-964.

М. Фарбер (M. Farber)

Поступила в редакцию

University of Durham, UK

01.07 .2008

E-mail: Michael.Farber@durham.ac.uk

Д. Шютц (D. Schütz)

University of Durham, UK

E-mail: dirk.schuetz@durham.ac.uk 